\title{
Primary and Secondary Flow Interactions in the Mixing Duct of a 2-D Planer Air Augmented Rocket
}

\author{
A Thesis \\ Presented to the Faculty of \\ California Polytechnic State University \\ San Luis Obispo
}

In Partial Fulfillment of the Requirements

For the Degree of Master of Science

In Aerospace Engineering

By

Martin Popish

May 2012 
(C) 2012

Martin Popish

ALL RIGHTS RESERVED 


\section{COMMITTEE MEMBERSHIP}

Title: Primary and Secondary Flow Interactions in the Mixing Duct of a 2-D Planer Air Augmented Rocket

Author: Martin Popish

Date Submitted: May 2012

COMMITTEE CHAIR: $\quad$ Dr. Dianne DeTurris, Professor

COMMITTEE MEMBER: Dr. Jin Tso, Professor

COMMITTEE MEMBER: Dr. David Marshall, Professor

COMMITTEE MEMBER: Mr. Ryan Gist, Industry Advisor 


\section{Abstract \\ Primary and Secondary Flow Interactions in the Mixing Duct of a 2-D Planer Air Augmented Rocket \\ Martin Popish}

Experiments were conducted on the Cal Poly air augmented rocket (AAR) in order to characterize two-dimensional flowfield phenomenon occurring in the mixing duct. The testing utilized a direct connect system where high pressure nitrogen is fed into the combustion chamber, to form a primary flow. The high pressure nitrogen is then expanded through a nozzle, with an area ratio of 22 and an exit area of $0.75 \mathrm{in}^{2}$, up to Mach 4.3. Secondary air is entrained from a plenum chamber which is used to create a lower stagnation pressure for the secondary flow. The two flows mix in a duct that has a cross sectional area of $2.06 \mathrm{in}^{2}$. The maximum pressure ratio, the ratio of primary to secondary stagnation pressure, achieved during testing was 132 . The stagnation pressures of the primary and secondary flows are transient throughout the test. The quasi-steady portion of each run increased with increasing pressure ratio. Pressure and temperature measurements were collected from ten test runs.

Shadowgraph images were taken of the mixing duct during testing in order to image the interactions between the primary and secondary flows. The images show an oblique shock forming in the primary flow. The angle of the shock matches theoretical predictions to within $8.41 \%$. The oblique shock begins at a distance of 1.5 inches downstream of nozzle exit when the AAR is operating in the Fabri choked condition. The images also show the mixing region which forms between the primary and secondary flows. The mixing region represents as much as $25 \%$ of the cross-sectional area of the flow field in the mixing duct two inches downstream of the nozzle exit.

An analysis of the secondary Mach number in the mixing duct shows that Fabri choking is occurring during testing. The secondary Mach number decreases as pressure ratio increases, in the Fabri choked condition. The transition to Fabri choking occurs at a pressure ratio of 100, suggesting that this is the pressure ratio of the saturated case.

The shape of the primary plume was compared to results from a 2-D simulation developed to predict the flow field inside the Cal Poly AAR. Although, the simulation is unable to predict the entire flowfield, modifications made it able to predict the velocity of the secondary, entrained, flow within $3.7 \%$. The modified simulation also predicts the that the primary plume will have expanded $98 \%$ of its total distance from the centerline of the mixing duct 1.7 inches downstream of the primary nozzle exit.

Pressure data taken along the wall of the mixing duct was used to identify the location of Fabri choking in the mixing duct. Tests showed that Fabri choking is occurring between 1 inch and 2.5 inches downstream of the nozzle exit. The location of Fabri choking moves farther downstream of the nozzle as pressure ratio increases. 


\section{Acknowledgments}

I'd like to thank everyone who supported me in completing this project. Most important on this list of people are my parents, Mary and Giles Popish. It has been their support and encouragement that have allowed me to accomplish this goal of mine. I do not know where I would be without their constant love and support.

I'd also like to acknowledge the excellent education I received at Cal Poly thanks to the amazing faculty and their tireless efforts to provide a quality education to their students. Thank you to my advisor Dr. DeTurris for providing me with guidance and direction throughout this project and my thesis committee for taking the time to review and critique my work. A special thanks goes out to Dr. Chen of the ME department who helped us use the ME department's high speed camera. He went above and beyond what I'd expect of a professor to assist in this project.

Finally I'd like to recognize all of those who have worked to make this project possible through their past and continued efforts. This project was made possible by the graduate work of Ryan Gist, Trevor Foster and Brett Morham and I thank them for laying the foundation for this work. I'd also like to give a special thanks to Josef Sanchez and Trevor Montre for working with me on this project; I would not have completed it without their help. Lastly I'd like to thank Jeffery Freeman for his work in continuing this project. 


\section{Table of Contents}

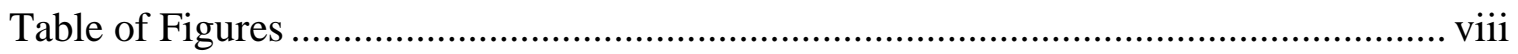

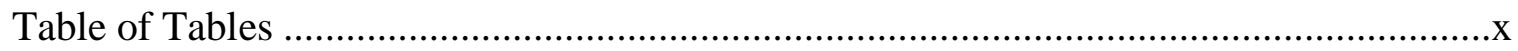

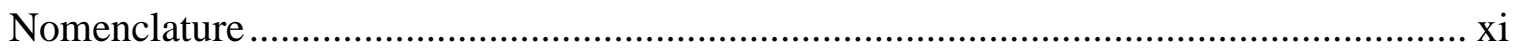

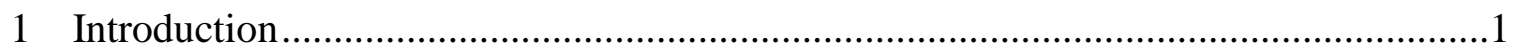

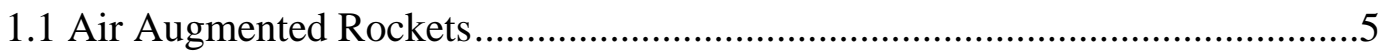

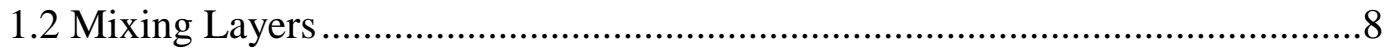

1.3 2-D Structure of Free Jets .................................................................... 10

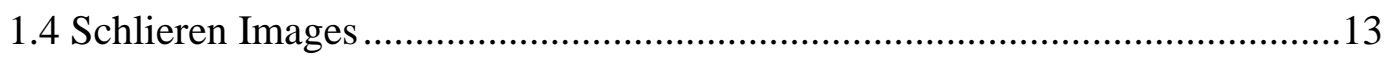

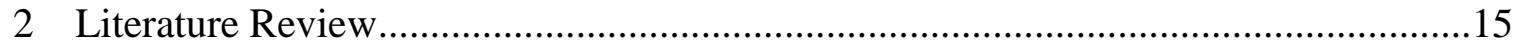

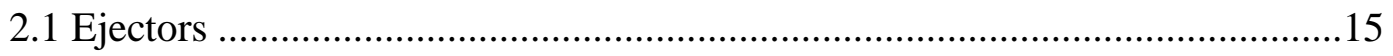

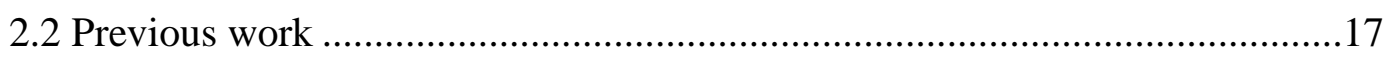

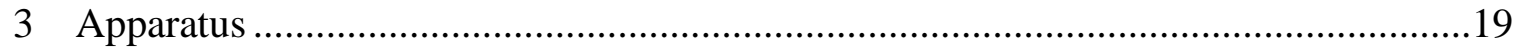

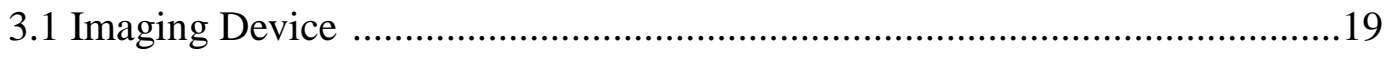

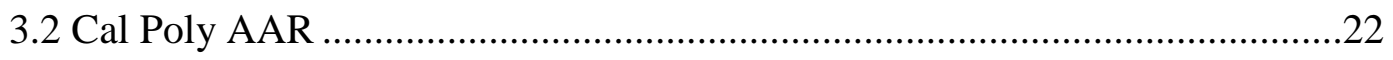

3.2.1 Rocket Nozzle .................................................................23

3.2.2 Bottom Plate..........................................................................24

3.2.3 Side Walls .......................................................................24

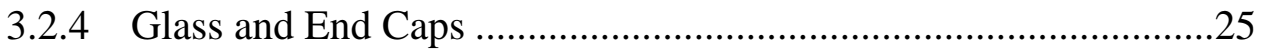

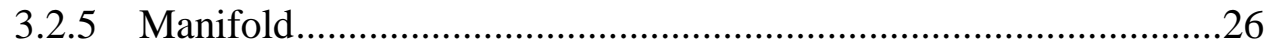

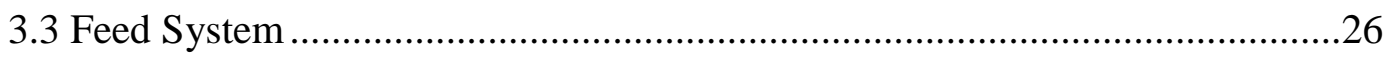

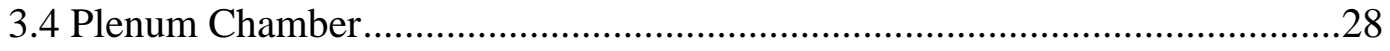

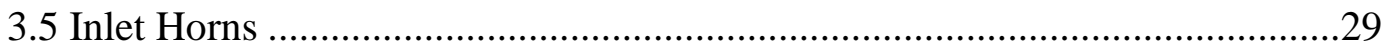

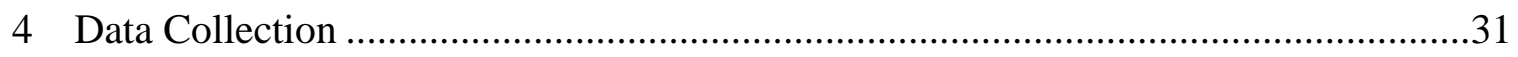

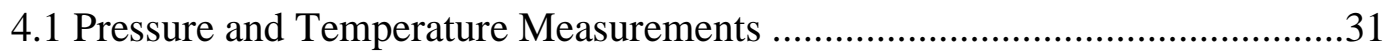

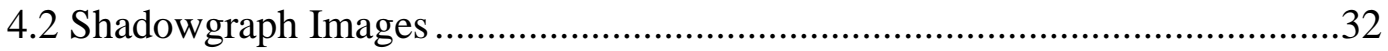

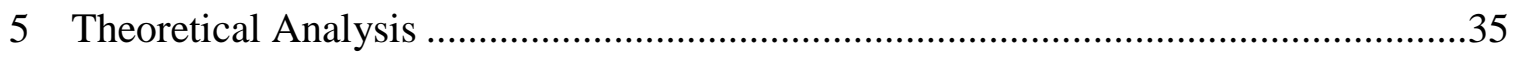

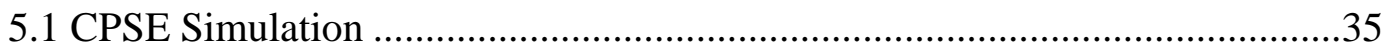

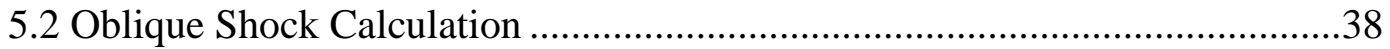




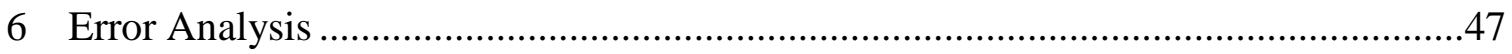

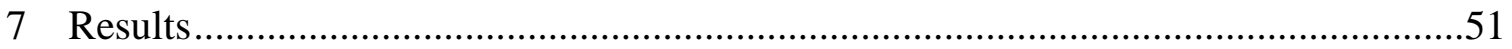

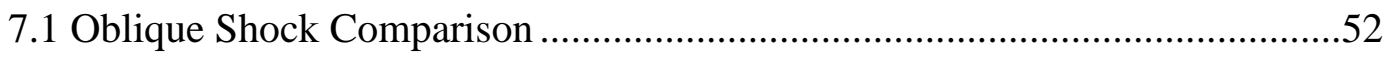

7.2 CPSE Simulation Comparison and Fabri Choking ...........................................64

7.3 Transient Considerations ..........................................................................

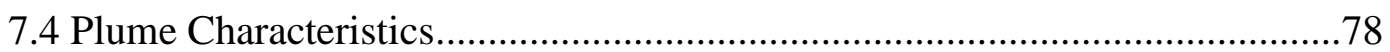

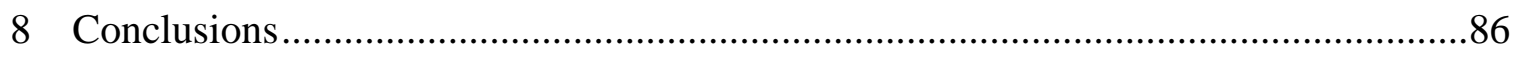

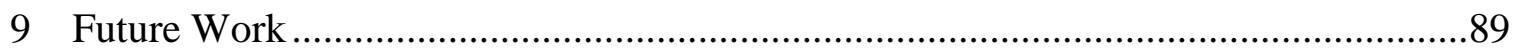

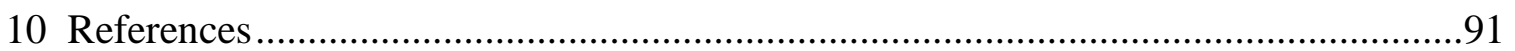

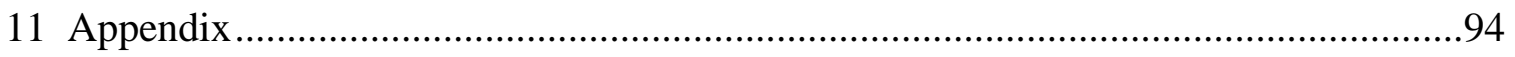

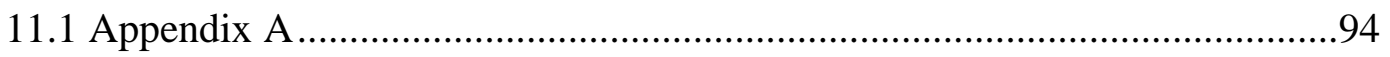

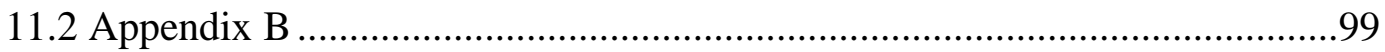

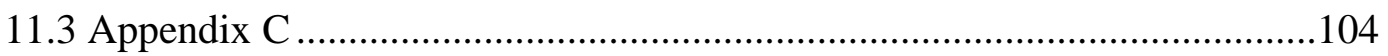




\section{Table of Figures}

Figure 1-1: Propulsive efficiencies of different engine cycles .........................................

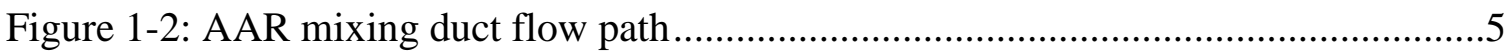

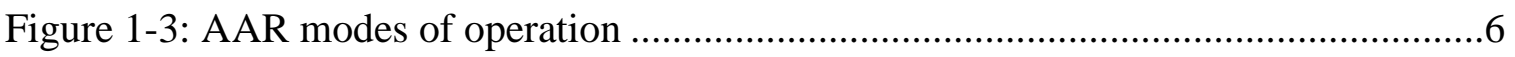

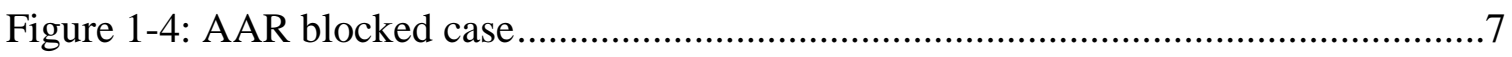

Figure 1-5: Initial velocity profile of a free shear layer .................................................

Figure1-6: Velocity profile downstream of jet entrance...................................................

Figure 1-7: Mixing layer growth in free shear flows...................................................10

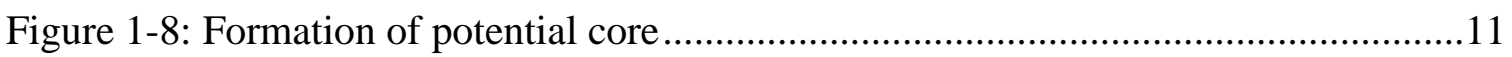

Figure 1-9: Periodic structure of a supersonic free jet...................................................12

Figure 1-10: Path of light through Schlieren apparatus ................................................14

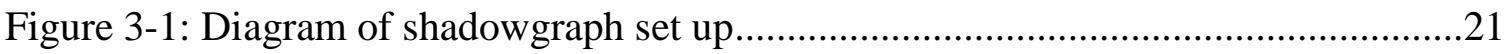

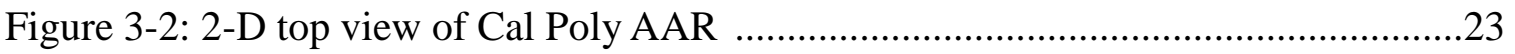

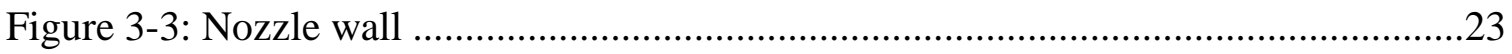

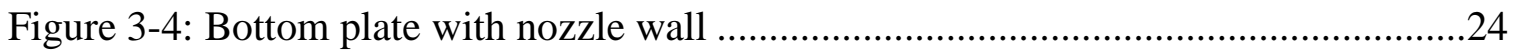

Figure 3-5: Assembly of bottom plate, nozzle wall, side wall and glass ..........................25

Figure 3-6: Front view of rocket manifold ................................................................26

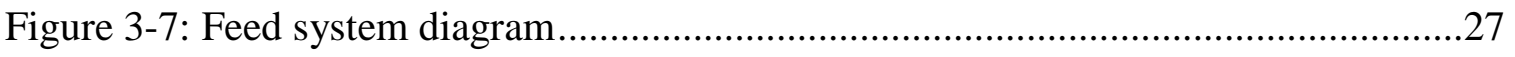

Figure 3-8: Interior of plenum chamber.................................................................28

Figure 3-9: (left) Plenum connected to manifold with flexible tubing;

(right) plenum connected to manifold with square tubing ....................................29

Figure 3-10: Inlet horns mounted inside of the plenum chamber …………….................30

Figure 4-1: Layout of measuring devices on the rocket ……………..............................32

Figure 4-2: Location of Schlieren image within the mixing duct ......................................33

Figure 5-1: Inviscid code prediction of primary plume expansion....................................37

Figure 5-2: Validation of CPSE simulation .................................................................

Figure 5-3: Under-expanded flow...............................................................................

Figure 5-4: Expansion Cycle …………………………….......................................42

Figure 5-5: Expansion fan and oblique shock prediction .................................................45

Figure 5-6: Over-expanded flow................................................................................

Figure 7-1: Flow field regions as seen in shadowgraph images .....................................52

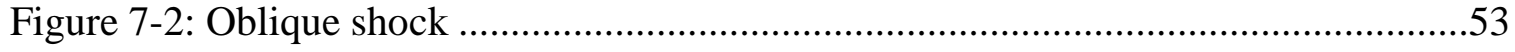

Figure 7-3: Points used for scaling image .................................................................54

Figure 7-4: Oblique shock data points ..........................................................................55 


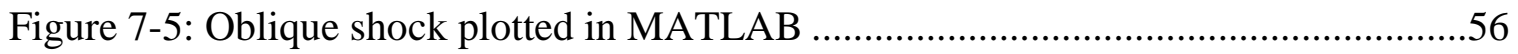

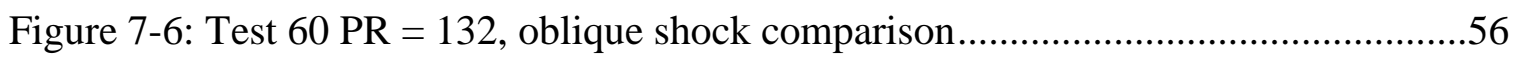

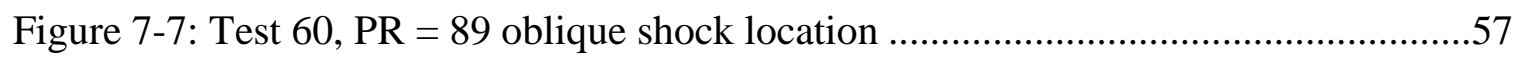

Figure 7-8: Wall pressure for Test $60 \mathrm{PR}=132$

compared to oblique shock observation..............................................................61

Figure 7-9: Test $60 \mathrm{PR}=89$, comparison of wall pressures and shock location ..............62

Figure 7-10: Oblique shock reflection off symmetry plane ..........................................63

Figure 7-11: Comparison of CPSE simulation to results from Test 60 at $\mathrm{PR}=132 \ldots \ldots . .64$

Figure 7-12: Simulation prediction of primary plume for Test 60 with a PR $=132$.......66

Figure 7-13: Comparison of Simulation and observed boundary layer

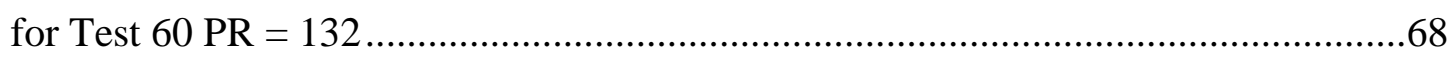

Figure 7-14: Comparison between simulation boundary and

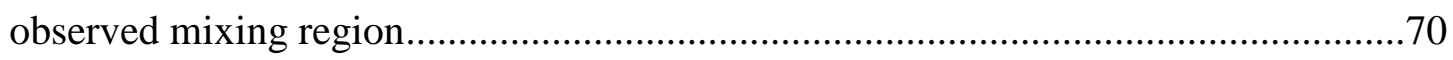

Figure 7-15: Secondary Mach number plots....................................................... 76-77

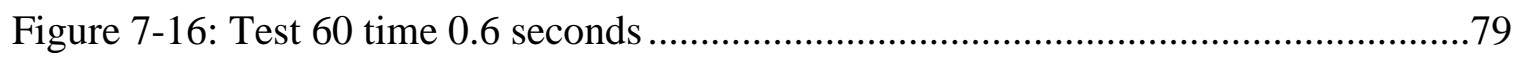

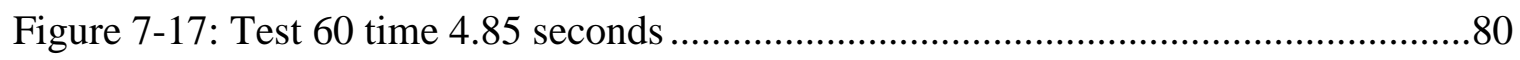

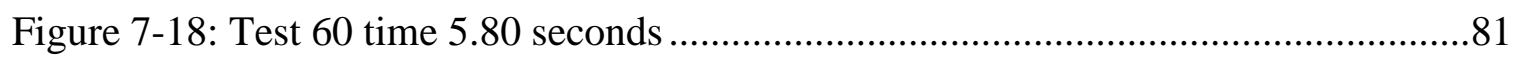

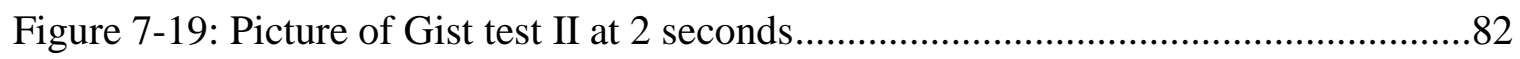

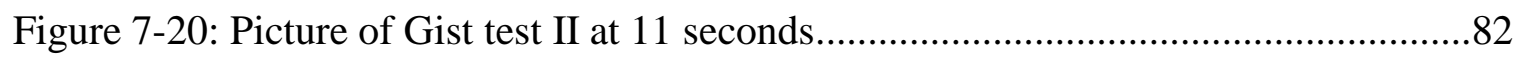

Figure 7-21: Test 53 (Left) time 0.29 seconds; Test 55 (Right) time 2.73 seconds .........83

Figure 7-22: Test 54 (Left) time 0.79 seconds; (Right) Test 56 time 3.45 seconds;

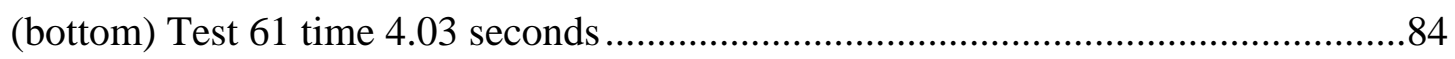

Figure 7-23: Test 57 (Left) time 0.13; (Right) Test 52 seconds time 0.75 seconds .........84

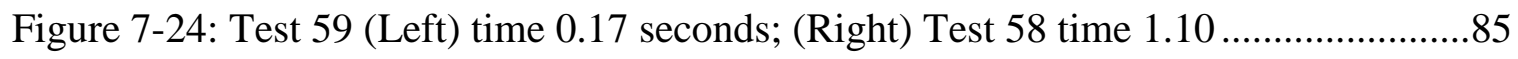




\section{Table of Tables}

Table 6-1: Percent error for key variables calculated using pressure measurements ........48

Table 6-2: Percent error associated with frame-data correlation ...................................49

Table 6-3 Breakdown of error for oblique shock angle ...............................................50

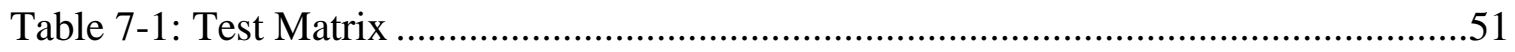

Table 7-2: Comparison of Oblique shock wave angles for Test 60 ...............................58

Table 7-3: Comparison of calculated and observed shock wave angles for Tests 52-61 ..59

Table 7-4: Comparison of Secondary Mach number between Test 60 and Simulation ....69

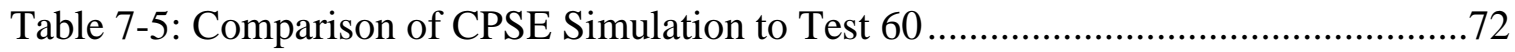




\section{Nomenclature}

$\begin{array}{lll}\mathrm{A} & \text { Area } & {\left[\mathrm{ft}^{2}\right],\left[\mathrm{in}^{2}\right]} \\ \mathrm{M} & \text { Mach Number } & - \\ \mathrm{M}_{\mathrm{n}} & \text { Normal Mach Number } & - \\ \mathrm{P} & \text { Stagnation Pressure } & {\left[\mathrm{lb}_{\mathrm{f}} / \mathrm{ft}^{2}\right]} \\ \mathrm{p} & \text { Static Pressure } & {\left[\mathrm{lb}_{\mathrm{f}} / \mathrm{ft}^{2}\right]} \\ \mathrm{R} & \text { Universal Gas Constant } & {\left[\mathrm{lb} f \mathrm{ft} / \mathrm{lb} \mathrm{m}^{\mathrm{o}} \mathrm{R}\right]} \\ \mathrm{T} & \text { Nozzle lip thickness } & {[\mathrm{in}]} \\ \mathrm{x} & \text { Distance from Nozzle Exit } & {[\mathrm{in}]} \\ \mathrm{y} & \text { Distance from Mixing Duct Centerline } & {[\mathrm{in}]} \\ \delta & \text { Expansion Angle } & {[\mathrm{degrees}]} \\ \varepsilon & \text { Oblique Shock Angle } & {[\mathrm{degrees}]} \\ \gamma & \text { Ratio of Specific Heats } & - \\ v & \text { Prandtl-Meyer angle } & {[\mathrm{degrees}]} \\ \rho & \text { Density } & {\left[\mathrm{slugs} / \mathrm{ft}^{3}\right]}\end{array}$

\section{Subscripts}

1

2

3

i

S

$\mathrm{t}$
Conditions at nozzle exit

Condition in the Primary flow after nozzle exit

Condition in the Primary flow before oblique shock

Arbitrary index denoting corresponding position

Secondary Flow

Conditions in the nozzle throat 


\section{$1 \quad$ Introduction}

The pursuit of new technology in the field of aeronautics has always been driven by the need to fly faster and higher. Starting with the breaking of the sound barrier and progressing on to the $\mathrm{X}-15$, the United States has given a significant amount of time and money to the venture of flying faster. There are many reasons for wanting to obtain increasingly higher velocities. The first reason is of strategic importance; faster systems allow our military to reach any location on the planet in shorter times. Secondly, from a commercial standpoint, inexpensive fast travel is desirable for moving people and valuable cargo around the world efficiently.

Today the new frontier for faster flight falls into the field of hypersonics. Hypersonic flight is defined as starting around Mach 5; however, there is no hard line that delineates hypersonic from supersonic flight ${ }^{1}$. Hypersonic flight holds many exciting promises such as rapid commercial transit, long range global strike capabilities and orbital launch vehicles. Hypersonic launch vehicles are of particular interest because of the promise they show for dramatically decreasing the cost of launching payloads into orbit. The increase in efficiency will come from the use of atmospheric oxygen, which will reduce the amount of oxygen that will need to be carried in tanks on the rocket ${ }^{1}$. Projects such as the National Aerospace Plane were aimed at achieving such a goal; unfortunately, the technology was not ready for such a demanding mission.

Today's explorations into hypersonics are focused on small scale demonstrators with the aim of advancing different components required for a fully integrated hypersonic vehicle. The focus of this thesis is on the propulsion systems of the hypersonic vehicle. 
In recent years, several hypersonic vehicles have been built and tested in order to better understand the different challenges associated with hypersonic flight. Perhaps the most prominent of these hypersonic technology demonstrators is the $\mathrm{X}-43$. The $\mathrm{X}-43$ was built to cruise for a short time at hypersonic speeds using a Scramjet propulsion system $^{2}$. The purpose of the experiment was to demonstrate the ability to start a Scramjet engine and control a vehicle in hypersonic flight. In January of 2004 the X-43 reached a speed of Mach 6.83 at an altitude of 94,069ft and underwent 10 seconds of powered flight $^{2}$. In November of 2004 the X-43 reached a speed of Mach $9.68^{2}$. It marked the fastest speed an air-breathing vehicle had ever reached.

Another important milestone was reached on May 26, 2010 when the X-51 made its first flight ${ }^{3}$. The $\mathrm{X}-51$ is another technology demonstrator developed to show the feasibility of a Scramjet engine. The X-51 is powered by standard jet fuel and is designed to cruise between Mach 6 and 7. During its first flight the X-51 cruised for 200 seconds at Mach $5^{3}$ which was slower than the speeds reached by the X-43; however, its 200 second flight time was the longest achieved by a Scramjet engine.

Scramjet technology continues to make strides as more and more tests are successfully conducted at hypersonic speeds. These tests provide valuable data and expertise on flight in the hypersonic regime. One drawback of these tests is that they rely on large booster rockets in order to accelerate the vehicle to hypersonic velocities. Making operational hypersonic vehicles a reality requires the means to fly through the subsonic and supersonic flight regimes without the need for a booster rocket. One solution to this problem is the combined cycle engine ${ }^{1}$. A combined cycle engine uses multiple engine cycles throughout its flight regime in order to operate more efficiently. 
Two different combined cycle engines have been proposed for hypersonic flight, the Turbine Based Combined Cycle (TBCC) and the Rocket Based Combined Cycle (RBCC). The TBCC was used in the SR-71. The SR-71 utilized a low speed turbine which is then bypassed at higher speeds, giving way to a Ramjet and then Scramjet engine ${ }^{4}$. The RBCC uses a rocket to accelerate the vehicle to supersonic velocities before being converted to a Ramjet, then Scramjet cycle. The TBCC has received a lot of attention for its merits in a hypersonic cruise vehicle because it has much better low speed performance and efficiency than the RBCC. The RBCC is considered the better choice for reaching orbit since the final stages of an orbital vehicle would require rocket power. Figure 1-1 below shows the potential of both the TBCC and RBCC propulsion systems, and their ability to operate more efficiently then rockets over a wide range of conditions.

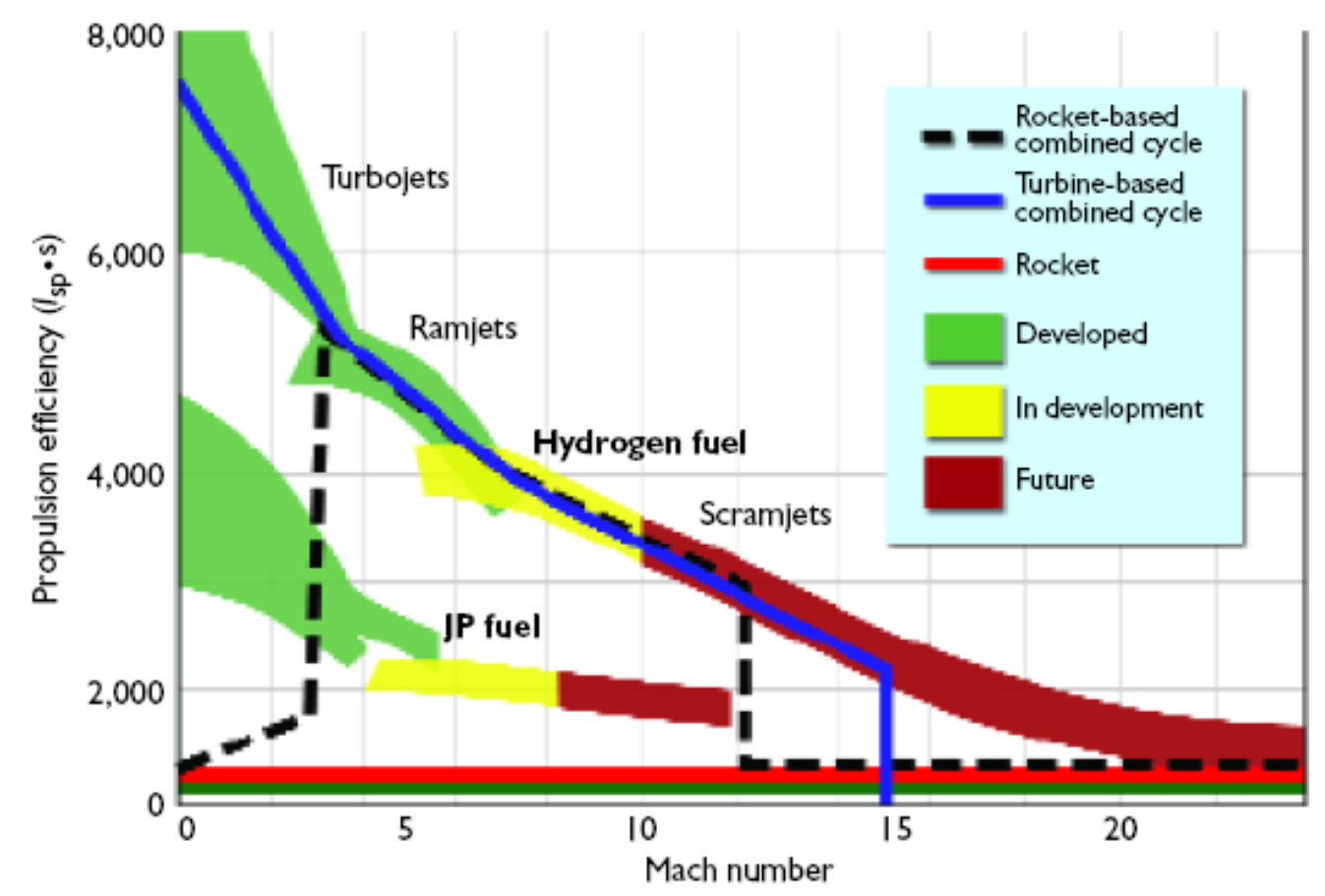

Figure 1-1: Propulsive efficiencies of different engine cycles ${ }^{5}$ 
The Rocket Based Combined Cycle system utilizes a number of different engine cycles over a large range of speeds, to efficiently accelerate a vehicle to high Mach numbers. The Air Augmented Rocket (AAR) is used as the first stage of an RBCC system, to accelerate the vehicle to between Mach 2.5 and Mach $3^{6}$; at this speed the AAR is replaced by a Ramjet cycle. The Ramjet cycle is much more efficient than the AAR cycle; however, it requires high supersonic speeds in order to operate effieciently ${ }^{6}$. Around Mach 6, the Ramjet cycle transitions to a Scramjet cycle, with the Scramjet taking the vehicle to higher hypersonic velocities ${ }^{6}$.

The RBCC is considered one of the best options for a next generation launch vehicle. It has a considerable ISP advantage over a conventional rocket launch vehicle, allowing for lighter cheaper systems ${ }^{6}$. The need for lighter, cheaper launch vehicles is the challenge that must be overcome in order to further develop orbital capabilities.

\subsection{Air Augmented Rockets}

Air Augmented Rockets, also known as ducted rockets, increase the efficiency of a rocket by increasing the mass flow rate of the rocket without adding extra propellant. The primary rocket exhaust expands into a duct where ambient air is entrained by the primary exhaust flow. Another feature that increases its efficiency is its ability to combine a fuel rich exhaust with ambient air to create an "afterburning" effect". The interaction between the primary and ambient flows must be understood in order to fully realize these benefits. Below, in Figure 1-2, is a diagram of an inviscid AAR flow path. 


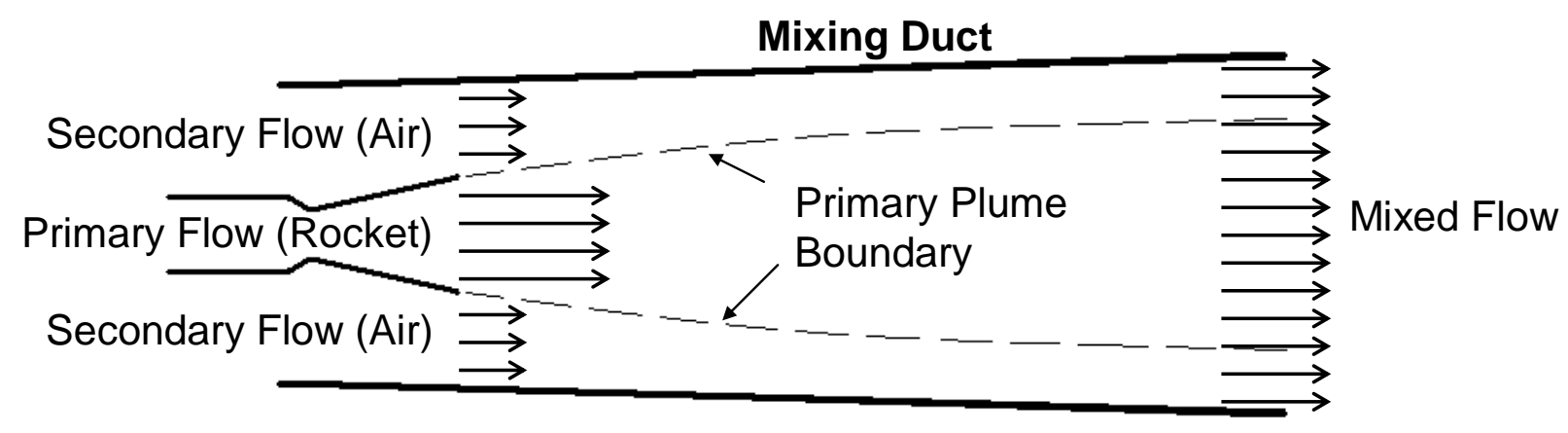

Figure 1-2: AAR mixing duct flow path ${ }^{5}$

The interaction between the primary and secondary flows is of great importance when designing an AAR. Depending on the nature of the two flows, there are several different modes in which the AAR can operate. If the primary flow is greatly overexpanded, the primary plume will become subsonic and mix with the subsonic air coming from the secondary duct at subsonic speeds. If the primary plume is optimally expanded, the secondary flow may reach sonic velocities at the entrance to the mixing duct and both flows will then remain supersonic through the mixing duct. If the primary flow is underexpanded, the primary plume will expand toward the wall causing the secondary flow to choke in the mixing duct. Figure 1-3 illustrates these three different modes of operation. 

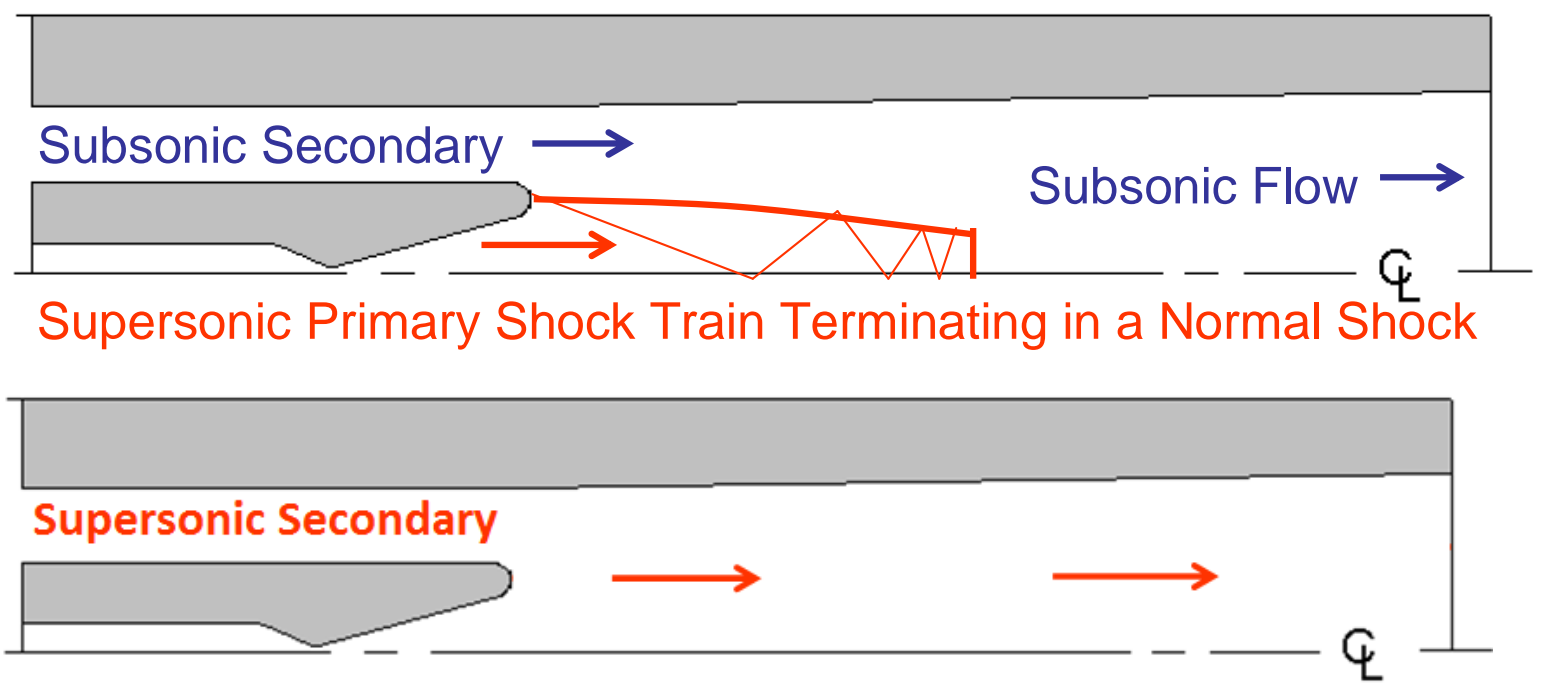

\section{Supersonic Primary}

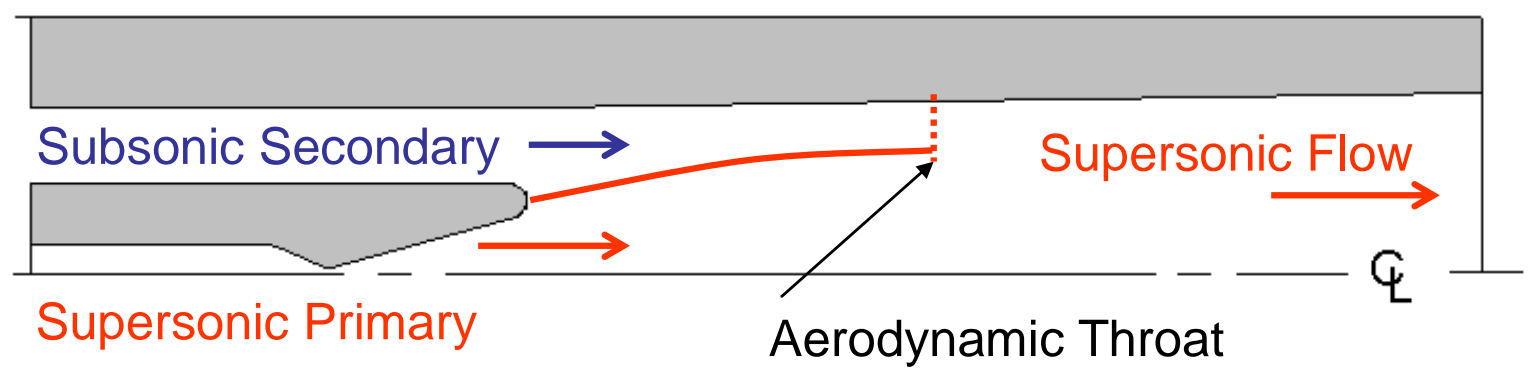

Figure 1-3: AAR modes of operation ${ }^{5}$

The ideal situation for AAR performance is to have the secondary flow choked at the entrance to the mixing duct. Choking in the mixing duct allows for the secondary flow to have the maximum possible area at its choke point, resulting in the highest possible secondary mass flow rate; a condition that is referred to as the "Saturated" condition $^{8}$. When the flow is choked in the mixing duct it creates a restriction on the secondary flow, limiting the mass flow rate. The condition where flow is choked in the mixing duct is termed "Fabri Choking" after Fabri who first described this flow phenomenon ${ }^{9}$. 
Another mode of operation that can occur in an AAR is the blocked case. The blocked case occurs when the primary plume is greatly under-expanded, and it expands to the walls of the mixing duct, completely cutting off the secondary flow. The blocked case should be avoided during AAR operation, because it causes the AAR to lose its augmentation. It is important to understand the conditions that cause the blocked case so that they can be avoided when designing an AAR. Figure 1-4 shows the flow path corresponding to the blocked case.

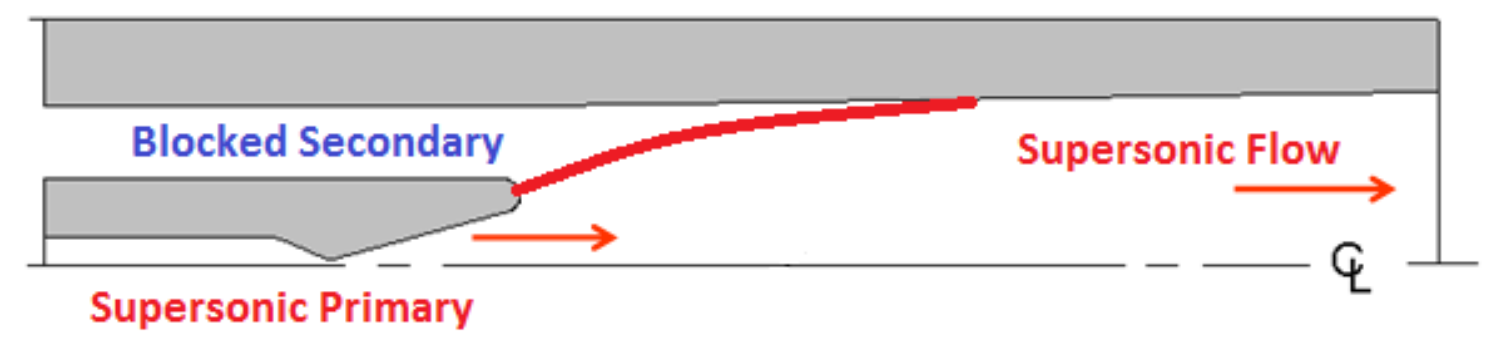

Figure 1-4: AAR blocked case

Understanding the AAR flow field and its limiting conditions, such as the blocked case, is imperative in designing an efficient $\mathrm{RBCC}$ engine. In an $\mathrm{RBCC}$ engine, the AAR, Ramjet and Scramjet all share the same flow path; meaning that the mixing duct of the AAR must be optimized for Ramjet and Scramjet operation as well as AAR operation. 


\subsection{Mixing Layers}

When describing the operation of an AAR it is important to take viscous effects into consideration. The boundary between the primary and secondary flows is not a thin line, but a growing shear layer. The shear layer, or mixing layer, is important because it represents an area of the flow field where re-combustion can occur. Under the right circumstances fuel rich primary exhaust can be re-combusted using oxygen from the secondary flow. In order to have efficient re-combustion the mixing layer must be understood.

Many studies have been done on shear layer growth between parallel flows. The basic results of these studies conclude that shear layers tend to grow linearly and are

primarily driven by the velocity and density differences between the two flows ${ }^{10}$. At high velocities compressibility effects cause a reduction in the rate of shear layer growth $^{10}$. The shear layer is the primary mechanism for the mixing of the primary and secondary flows. Understanding the parameters that contribute to shear layer growth is necessary to understanding the amount of mixing occurring between the primary and secondary flows.

For a better understanding of what is occurring between the primary and secondary flows, consider the simple case of two parallel flows of different velocities. Figure 1-5 below gives an illustration of two parallel flows. 


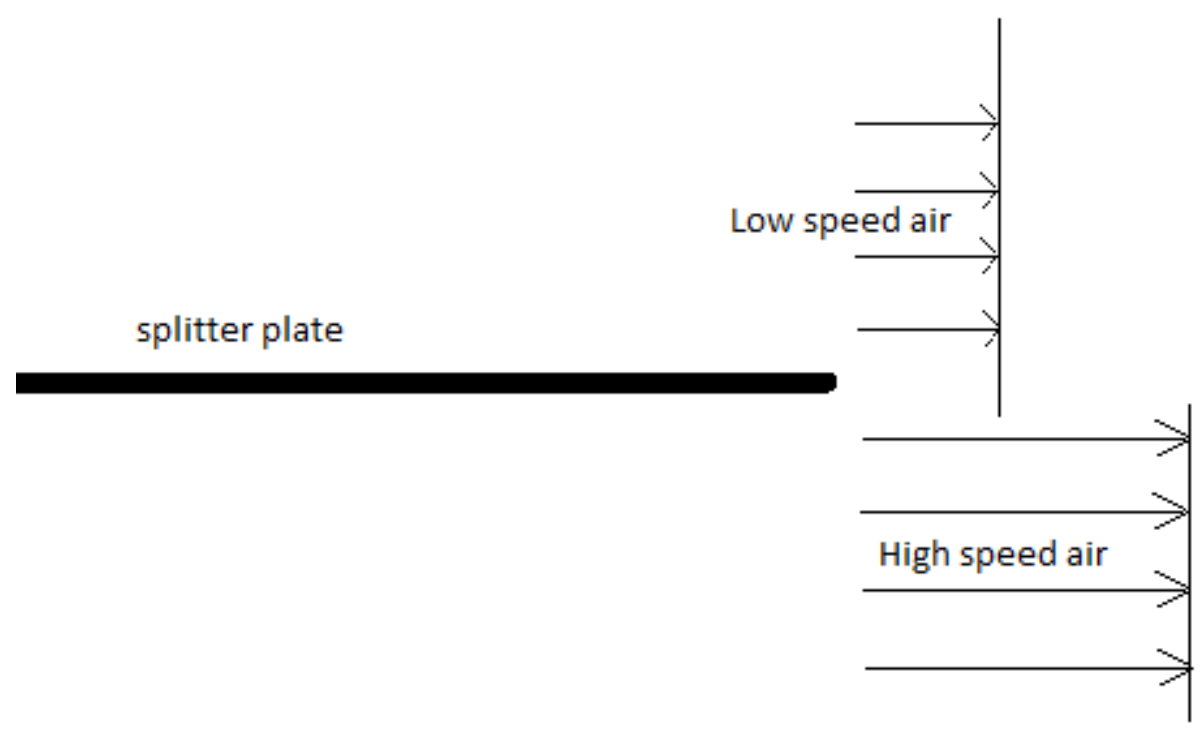

Figure 1-5: Initial velocity profile of a free shear layer

Notice there is an abrupt change in the velocity from one stream to the next.

Physically, the abrupt change in velocity is not sustainable. Viscous effects will cause a smooth transition in velocity between the two flows. Shear forces between the two streams cause one to slow down and the other to speed up causing a turbulent interface, or boundary, between the two flows ${ }^{11}$. An illustration of the change in velocity profile can be seen in Figure 1-6.

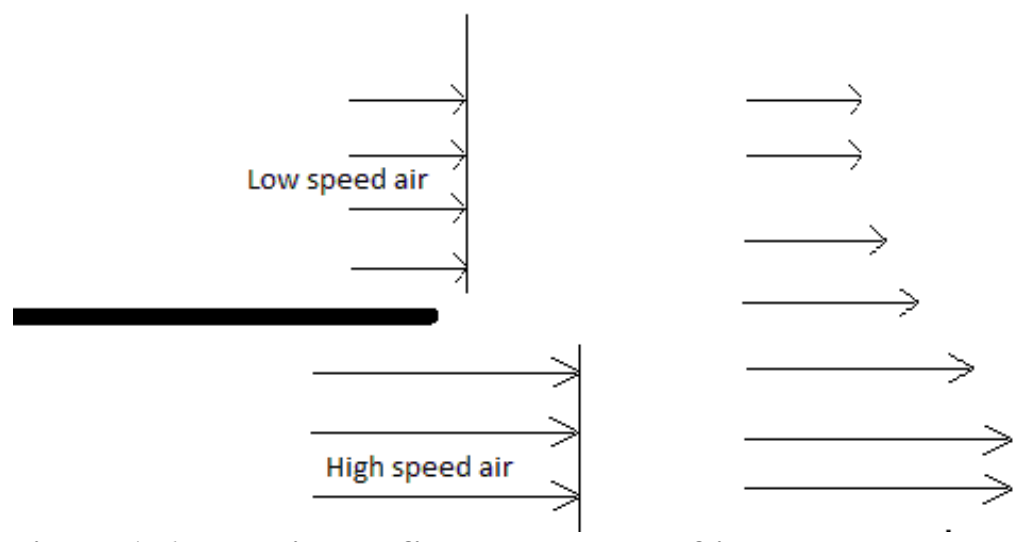

Figure 1-6: Velocity profile downstream of jet entrance 
The thickness of the boundary tends to grow linearly as the flow moves downstream taking on a wedge shape ${ }^{11}$. Figure 1-7 shows what the shear layer looks like when viewed using high speed cameras and a Schlieren imaging system. Notice that the turbulent layer between the two flows grows linearly as it moves downstream. Also notice the edge of the shear layer is not smooth; the turbulent nature of the mixing region causes its boundaries to be unsteady.

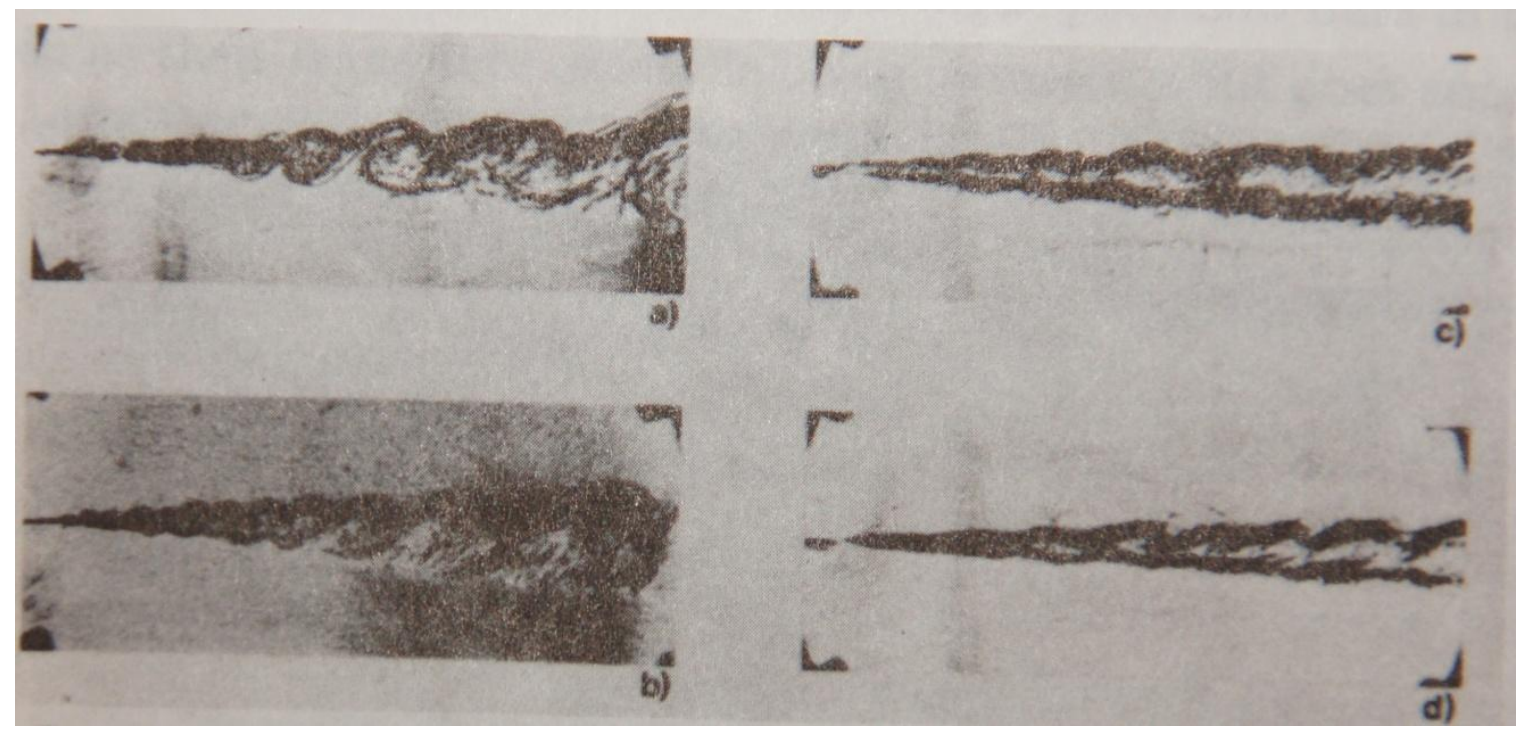

Figure 1-7: Mixing layer growth in free shear flows ${ }^{10}$

\section{$1.3 \quad$ 2-D Structure of Free Jets}

In order to apply the concept of shear layers derived from parallel flows to an AAR we must look at the case of a free jet; where the jet flow is exhausted into a stationary flow field. In the near field, right at the jet exit, there are two separate regions that are formed. At the centerline of the jet, there is a potential core where the flow is 
uniform $^{12}$. The flow inside the potential core has not been slowed down by viscous effects. When looking at Figure 1-6, one will notice that the arrows at the bottom are still at their full length, showing that they have not slowed down. The center of the flow is still at its initial velocity because the viscous effects spread out slowly from the boundary between the flows. In a jet the potential core corresponds to the area along the center of the jet, which is initially unaffected by viscous forces. Outside of the potential core is the shear layer, which is created by the velocity gradient between the two flows ${ }^{11}$. The shear layer acts much like the shear layer formed by the parallel stream described above.

After a distance of about 4 to 5 jet diameters downstream, the potential core is consumed by the growing shear layer ${ }^{11}$. At the end of the potential core the shear layers developing on the edge of the jet flow merge at the centerline of the jet ${ }^{13}$. An illustration of the merging boundaries is shown below, in Figure 1-8.

low speed air

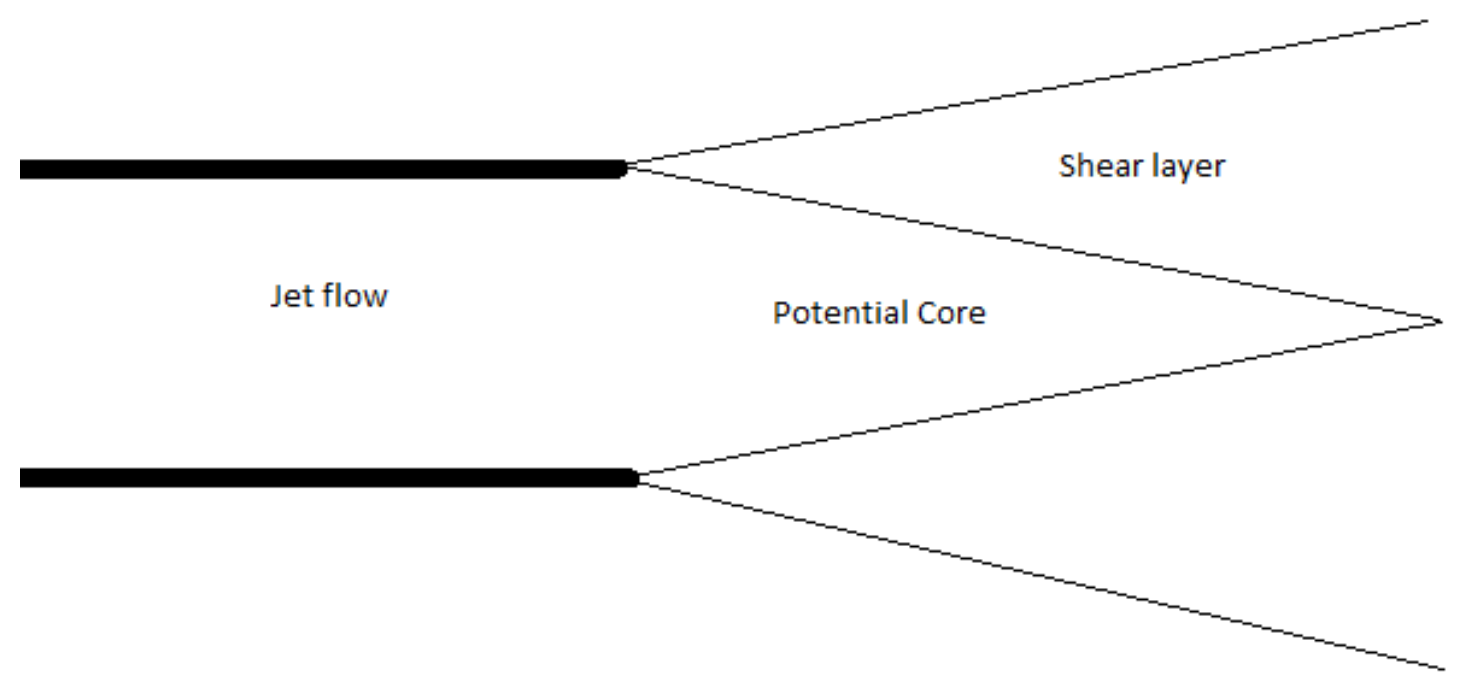

Figure 1-8: Formation of potential core 
Much of the research done on free jets involves the use of subsonic jets in a stationary field. An analysis of free jets helps give insight into the flow field of an AAR, but it does not give the entire picture. In an AAR, the jet is supersonic and supersonic jets have a periodic structure ${ }^{11}$. The periodic structure consists of a series of shock and expansion waves which accelerate and decelerate the flow. Numerical methods such as the Method of Characteristics must be used in order to solve for the structure of the jet ${ }^{11}$. Figure 1-9 shows the periodic structure of a supersonic jet for the cases of underexpanded and over-expanded flow.

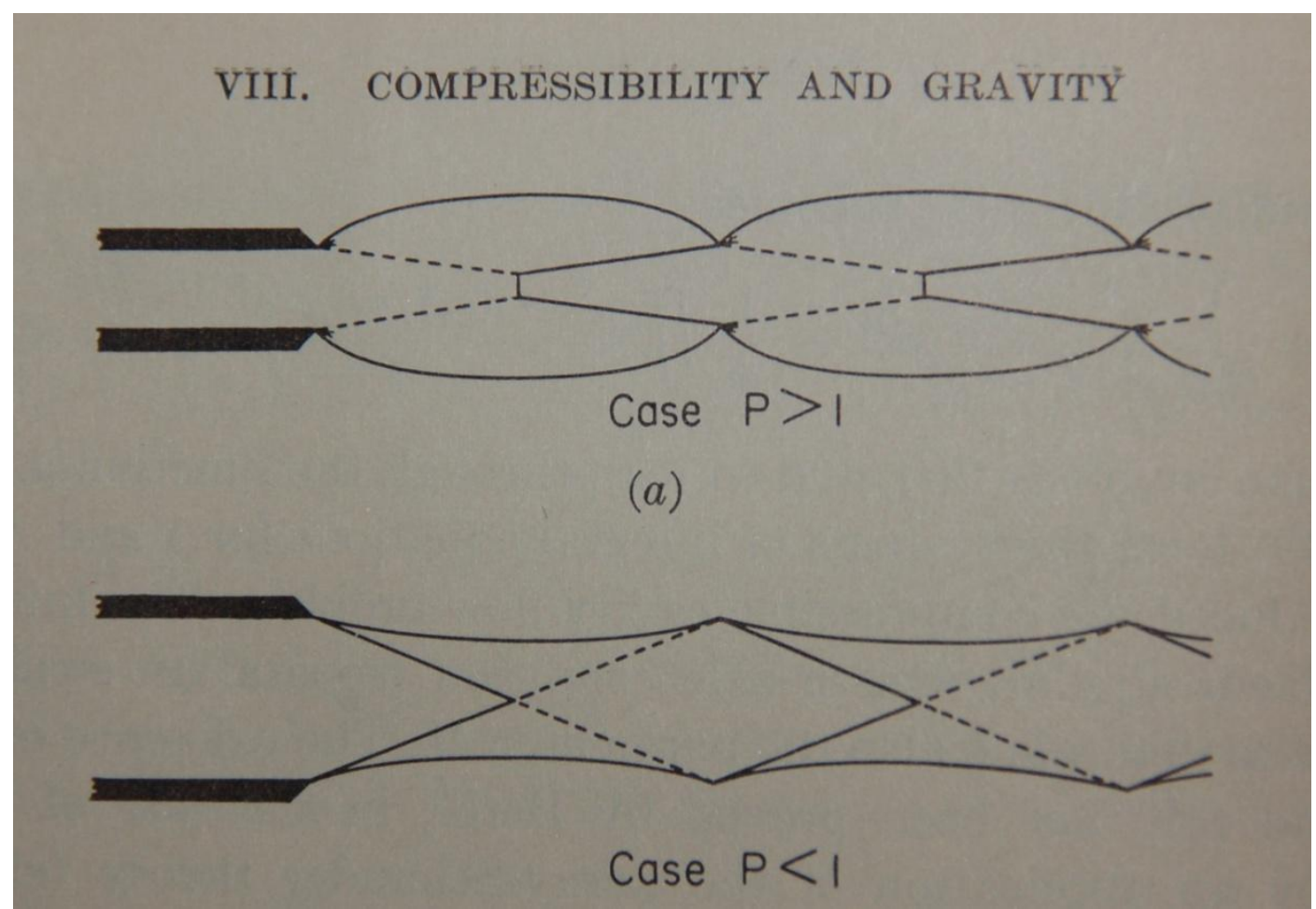

Figure 1-9: Periodic structure of a supersonic free jet ${ }^{11,12}$

When the flow is under-expanded, it expands out, and then is turned back towards the centerline by an oblique shock. After being collapsed by the shock the flow expands back out; creating a cycle that repeats itself as the jet flow moves downstream. In the 
over-expanded case, the jet flow collapses down before expanding back out; creating the classic "shock diamond" that is often seen in large rockets at launch.

\subsection{Schlieren and Shadowgraph Imaging}

Two key pieces of technology that were used in this experiment are a Schlieren and shadowgraph imaging system. "Schlieren" simply means "streaks" named after the streaks and striations that appear in a Schlieren image ${ }^{14}$. The first person to discover these images in an experimental setting was Robert Hooke, back in the late 1600's. He produced a Schlieren image of a candle with the heat waves traveling up from it. Scientists of Hooke's generation found very little use for the images, and the technology was largely forgotten. In the $19^{\text {th }}$ century, a scientist named Foucault came up with what is known as the knife-edge test to check for imperfections in astronomical telescope mirrors $^{14}$. The knife-edge test was then rediscovered by German scientist August Toepler in the development of the first modern Schlieren techniques ${ }^{14}$. It was Toepler who named the technique Schlieren. The method relies on the fact that density variation in the air cause light to bend as it passes through these variations. The original Schlieren technique, devised by Toepler, used lenses and a knife-edge to image these variations ${ }^{14}$. A point light source would be projected through the first lens and onto the object of interest. The light would then be focused onto a knife-edge using a second lens. As the air density changes around the object, the light is bent either into or over the knife-edge causing a light-dark contrast that forms the Schlieren image ${ }^{14}$. Figure 1-10 shows a diagram of the light as it passes through our Schlieren system. One thing to note is that 
our Schlieren system uses mirrors instead of lenses. Light is bounced off one mirror and through the test section before being focused to a point by the second mirror.

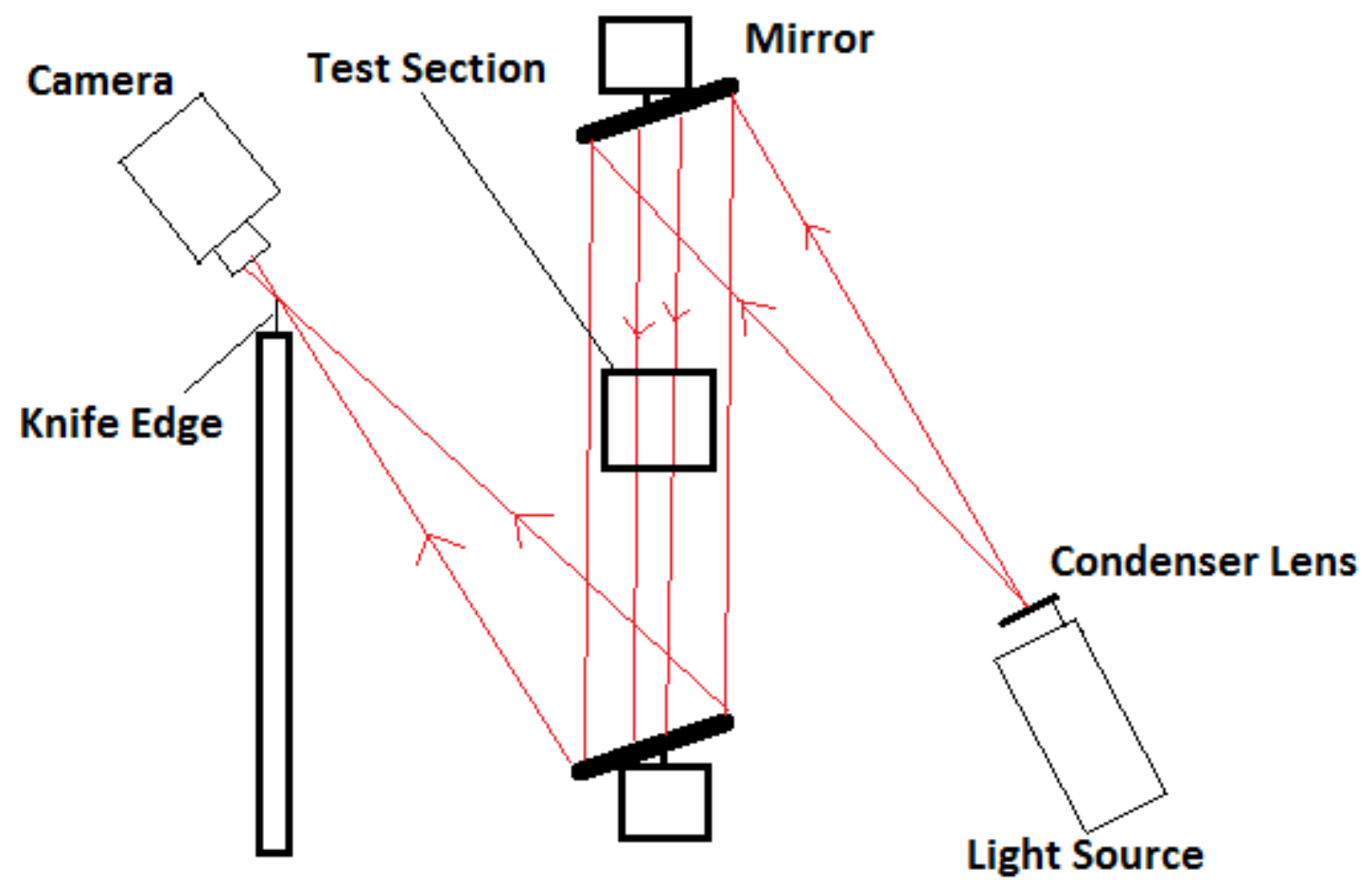

Figure 1-10: Path of light through Schlieren apparatus

A shadowgraph is similar to a Schlieren in that it helps image density variations in a flow field. The difference is that the image is created from the shadow caused by the density variations rather than by the bending of light into or around the knife-edge ${ }^{14}$. The shadowgraph uses the exact same mirror configuration as the Schlieren system, the only difference is that the knife-edge is removed and the image is projected directly onto a surface, where it can then be filmed. When a shadowgraph is aligned so that parallel light is being sent through the test section it is called focused shadowgraphy ${ }^{14}$. 


\section{Literature Review}

The following section contains a review of relevant research in the fields of supersonic ejectors as well as previous AAR research that has been conducted here at Cal Poly.

\subsection{Ejectors}

Fabri and Siestrunck analyzed the performance of supersonic ejectors in the late 50's. Their analysis centered on calculating performance metrics and determining the driving factors in optimal performance. Their research analyzed high pressure ratio ejectors with supersonic primary flow ${ }^{15}$. The performance of a given condition was determined based on the ratio of secondary mass flow entrained by the ejector to primary mass flow ejected through the nozzle, termed the entrainment ratio. A higher entrainment ratio implies more efficient performance ${ }^{15}$. Another important operational point occurs when the maximum amount of secondary mass flow is achieved. A condition Fabri terms the saturated condition. When operating in the saturated condition the secondary flow reaches Mach 1 at the entrance to the mixing duct ${ }^{15}$. In Fabri's tests the back pressure of the secondary flow was held constant so the mass flow in the secondary duct is limited only by available area when choked flow is achieved. Choking at the exit of the mixing duct represents the largest available area for the secondary flow to be choked. The saturated condition is only a function of ejector geometry and pressure ratio, the primary and secondary streams remain distinct ${ }^{15}$. 
Fabri and Paulon used an axisymmetric ejector to test performance in supersonic ejectors. When the stagnation pressure of the primary flow is sufficiently large, the primary flow expands and impinges on the secondary flow, a condition known as "Fabri choking"'. When the secondary flow chokes in the duct; the mass flow of the secondary flow is limited by available duct area.

Addy's study of axisymmetric ejectors was centered on modeling the flow phenomenon occurring in the ejector. Previous supersonic ejector analysis was dependent on 1-D or quasi 1-D analysis. These techniques were able to predict ejector performance for some cases, but were limited in their use. These methods of analysis could not account for non-constant area mixing and variable ejector length. Addy proposes a 2-D solution using Method of Characteristics to model the primary flow and a 1-D method for modeling the secondary flow ${ }^{8}$. Addy also gives suggestions for modeling viscous interactions within the flow. One of the suggested methods is viscous superposition. Viscous superposition involves creating an inviscid solution and super imposing a shear layer model into the flow ${ }^{8}$. Addy also explores the differences between a steady state and transient solution.

Papamoschou conducted an analytic study comparing the performance of axisymmetric ejectors to 2-D ejectors. Papamoschou used a quasi 1-D method which incorporated shear layer models, in order to account for shear stress between the primary and secondary streams, as well as the wall shear stress ${ }^{16}$. Papamoschou's analysis determined that an axisymmetric configuration gave greater thrust augmentation than the 2-D configuration. The increased performance is due to the decreased wall shear stress in the axisymmetric case. 


\subsection{Previous work}

Trevor Foster initiated AAR testing at Cal Poly by constructing a 2D planer, AAR developed to study Fabri choking. The test used a methane and oxygen rocket to produce the primary exhaust. Methane and oxygen were injected into a combustion chamber with a width of 0.5 inches and a length of 2.5 inches ${ }^{17}$. The tests utilized primary combustion pressures ranging from 325 to 1032 psi resulting in a maximum pressure ratio of $74^{17}$. During testing the combustion chamber was cooled by pumping water through cooling channels in the chamber and nozzle walls. Ambient air was entrained through square ducts that drew the flow in through inlet horns. The results of these hot fire tests were correlated with results from cold fire tests to see if any similarities exist. Initial findings indicated that Fabri choking was occurring in roughly the same location for both cold and hot fire tests.

Ryan Gist used the Cal Poly AAR rocket to explore Fabri choking and understand the relationship between pressure ratio and entrainment ratio. Gist's tests used high pressure nitrogen to drive the primary flow; the secondary flow was entrained from ambient conditions. Gist was able to correlate real entrainment ratio data taken from the test along with results from a quasi-1D analysis in order to improve the predictive capability of that analysis ${ }^{5}$. The correction was done through the use of a variable termed $\mathrm{K}_{\text {expand. }}$ The $\mathrm{K}_{\text {expand }}$ term takes into consideration the non ideal nature of the predicted expansion fan. Gist's experiments showed the value of $\mathrm{K}_{\text {expand }}$ to be 1.5 for the Cal Poly $\mathrm{AAR}^{5}$. Gist also discovered that the 2-D configuration is vulnerable to asymmetric flow 
conditions between the two secondary inlets, allowing them to operate in different modes at the same time ${ }^{5}$.

Brett Morham wrote a computer code, the CPSE simulation, which predicts the flow field characteristics in the mixing duct of the AAR up to the Fabri choke point. The CPSE simulation is an inviscid approximation and is developed using the 2-D method proposed by Addy. The simulation was created in order to analyze the effect of different configuration changes on entrainment ratio. It provides pressure, temperature and velocity measurements throughout the flow field. The results produced by the simulation match up with experimental entrainment ratio data within $4 \%{ }^{18}$. These results suggest that entrainment ratio is driven almost entirely by inviscid pressure forces within the flow. The CPSE simulation also predicts the pressure ratios corresponding to different modes of AAR operation for a given geometry. For the geometry of the Cal Poly AAR it was found that a saturated case would occur at a pressure ratio of $70^{18}$. The rocket is predicted to reach a Fabri blocked case at a pressure ratio of $230^{18}$. 


\section{Apparatus}

The following section outlines the various hardware components used to collect the data for this paper. The major components are the imaging system and the AAR rocket. Various measuring devices were also used to collect and record data.

\subsection{Imaging Device}

For these tests a Schlieren imaging device, originally meant for viewing the $\mathrm{Cal}$ Poly supersonic wind tunnel, was modified in order to fit with the AAR hardware. The original hardware was constructed in such a way that the light from a point source was completely contained within a series of PVC pipes. The pipes allowed the device to be operated in less than perfect darkness. It also held the mirrors at large angles which maximized defects that are inherent in Schlieren images. In order to reduce the angle the mirrors were removed from the PVC pipe and mounted around the test section in a Ztype configuration. The Z-type configuration eliminates coma, one of the two defects associated with a mirror-Schlieren system ${ }^{14}$. Coma results in the spreading out of the light source from a point into a line, with one region being illuminated more than others. In a Z-type configuration the opposing angle of the two mirrors cancel out the spreading effect that occurs when a point light source is bounced off an angled mirror. The other defect that occurs is called astigmatism, which causes the image to spread out in the

cutoff plain, making it difficult to focus the image ${ }^{14}$. Astigmatism can be reduced by reducing the angle of the mirrors and increasing the focal length of the mirrors. Both of these solutions cause the mirrors to be farther apart. Due to space limitations we were 
only able to reduce the angle of the mirrors to 20 degrees. As a result astigmatism made it very difficult to focus the Schlieren image. Another factor that made focusing difficult was that the mirrors are hanging in space as opposed to being set up on a level plane. The mirrors are suspended from a fixed stand that only gives a limited capacity to move and adjust to mirrors' location. As a result it was impossible to perfectly align the mirrors, and the image was out of focus near the cutoff plane. The fact that the image was not well focused near the cutoff plane made it impossible to capture a clear Schlieren image with the camera and lenses we had available. As a result the decision was made to use a less sophisticated, but equally useful imaging method, the shadowgraph technique. In order to convert the Schlieren to a shadowgraph the knife-edge was removed from the set up and a piece of tracing paper was placed near the second mirror. The image bouncing off the second mirror was projected onto the tracing paper. Because the tracing paper is very thin the image could be seen clearly on the other side of the paper. The camera was placed behind the tracing paper and was focused onto the image projected on the tracing paper. Figure 3-1 is a diagram of the shadowgraph set up used during testing. 


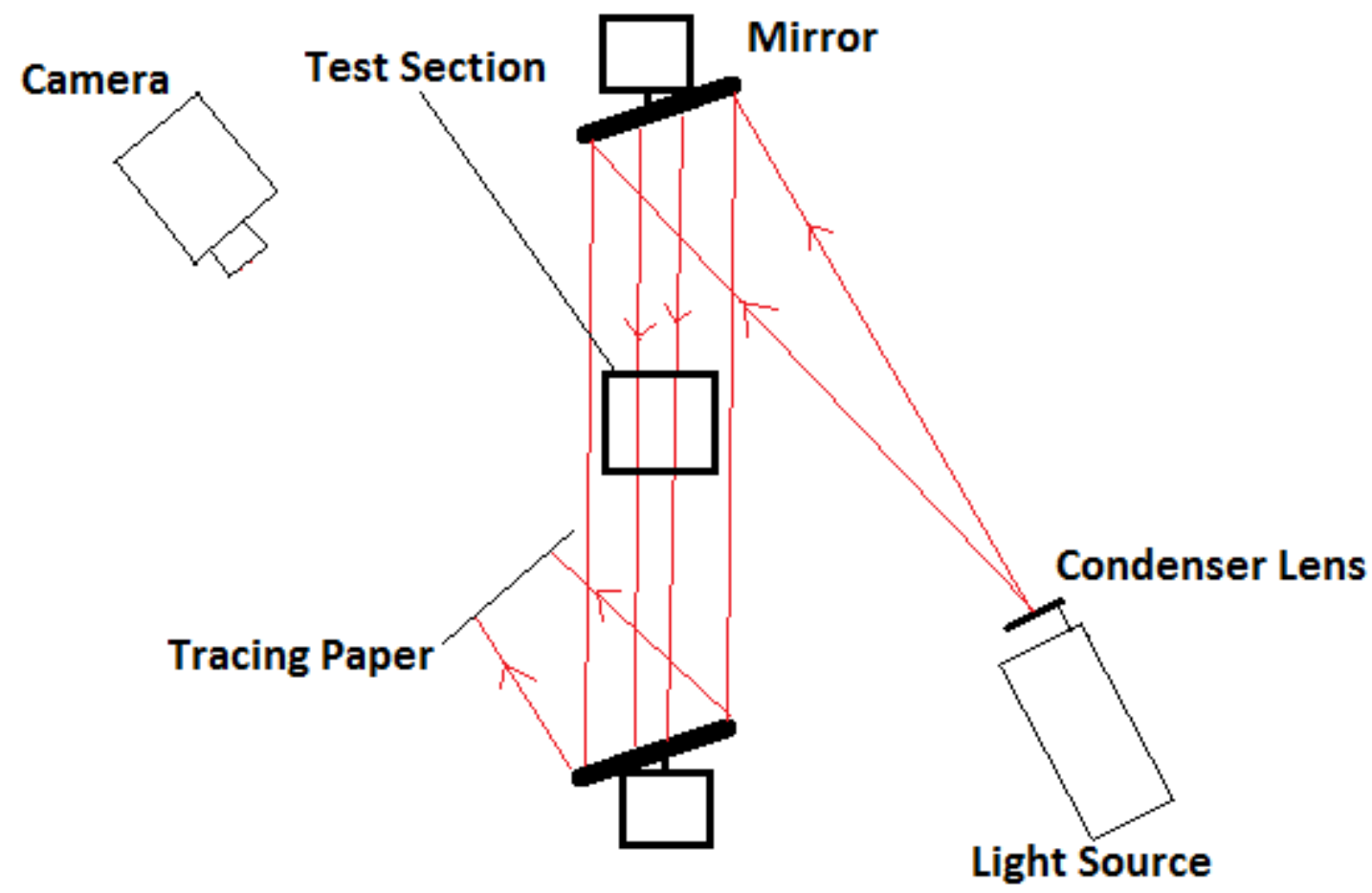

Figure 3-1: Diagram of shadowgraph set up

The mirrors used in the shadowgraph are spherical F10 mirrors with a 1/8 wave accuracy and are over coated with silicone monoxide. The mirrors have a 6" diameter and a 60 " focal length. The stand used to mount the mirrors consists of a 6 " square steel tube that is mounted to the floor of the test cell. The main support extends 8 ' 3 " high and has two three foot support arms attached to it. The arms extend out above and below the test section and provide a solid structure to attach the mirrors. The mirrors are attached to the arms using two U-brackets, which allow the mirrors to be rotated in two different axes. The light source is an Olympus model CLV-A 300 Watt xenon short-arc lamp. The lamp has a maximum luminous flux of 6000 lumens. In order to ensure that the light is focused to a point a condenser lens was placed over the light source. The condenser lens is $50 \mathrm{~mm}$ in diameter and has a focal length of $44 \mathrm{~mm}$. The camera used to record the images is a Vision Research Phantom v310 high speed camera. The camera has an image 
clarity of 1 megapixel and is capable of recording up to 500,000 frames per second (fps). A 50mm Canon camera lens was used to focus the camera onto the viewing surface.

\subsection{Cal Poly AAR}

Several years ago an AAR rocket was built in order to explore the different flow phenomenon occurring in the mixing region of an AAR. The rocket produces a supersonic primary flow which is used to entrain air from the ambient environment. It was built to run cold flow tests and hot fire, reacting flow, tests. The tests explored in this thesis are only cold flow tests and do not necessitate hot fire capability.

The AAR used for these tests is a modified version of a rocket that was used several years ago. The current rocket uses the same nozzle, manifold and upper chamber plate as the original rocket. The bottom plate was reconstructed in order to allow for glass to be placed on the top and bottom of the mixing duct. The glass allows for shadowgraph images to be taken. The mixing duct was also lengthened in order to reduce the affects of the exit pressure on the mixing region. Figure 3-2 shows the layout of the rocket. 


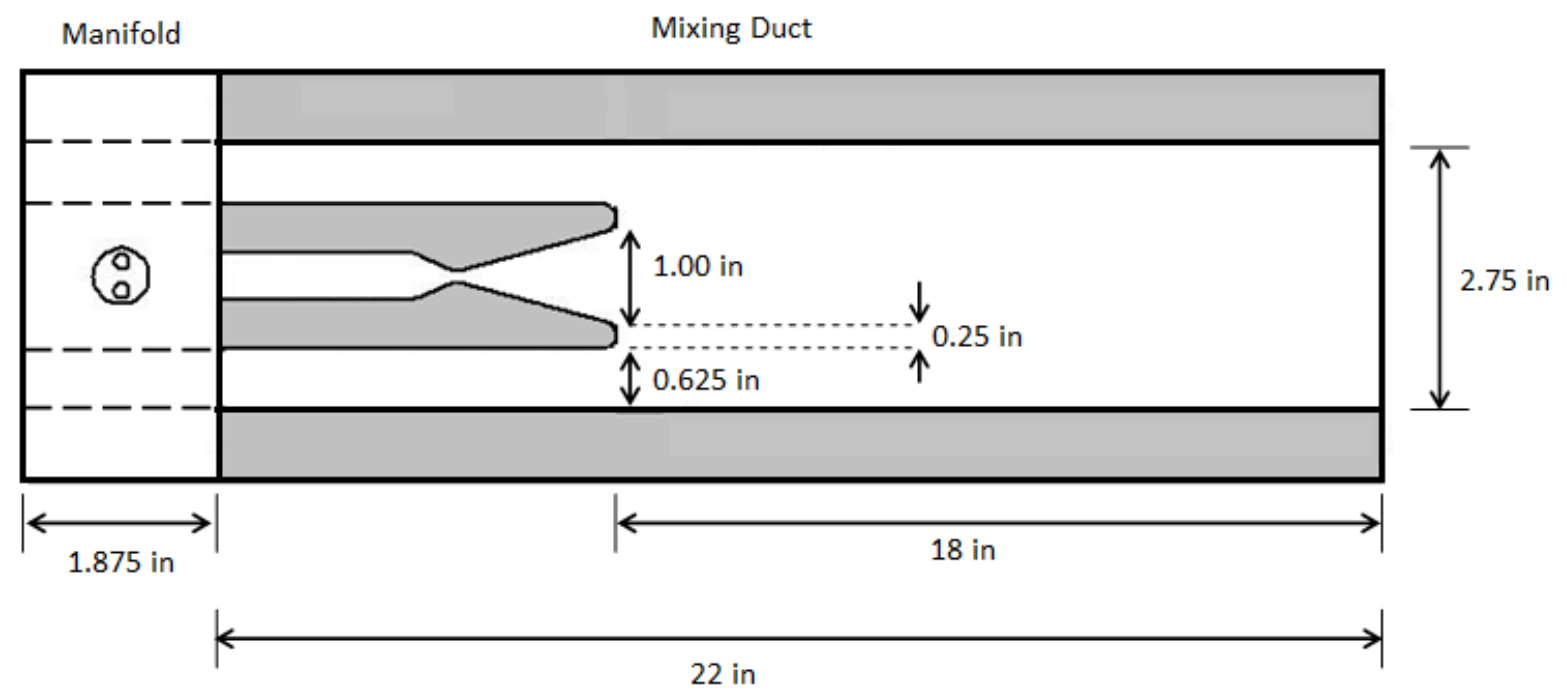

Figure 3-2: 2-D top view of Cal Poly AAR (drawing is not to scale)

\subsubsection{Rocket Nozzle}

The nozzle used for these tests has a straight wall angle of 15 degrees with an area ratio of $22^{17}$. The walls of the nozzle have cooling channels in them in order to accommodate hot fire testing. These cooling channels were not used during these tests. The nozzle has a design exit Mach number of about 4.8; however, its actual exit Mach number is approximately Mach 4.3. Figure 3-3 shows a picture of one half of the nozzle.

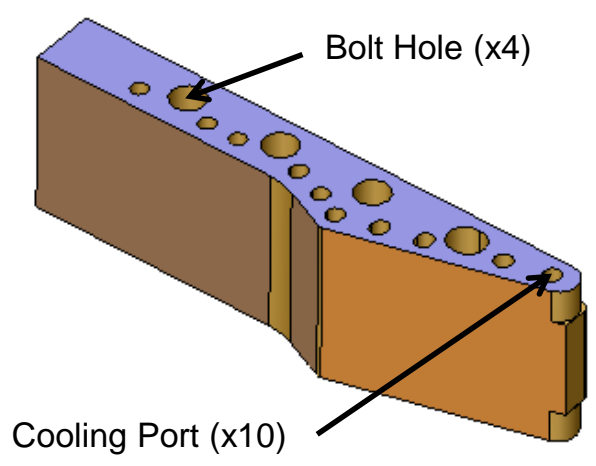

Figure 3-3: Nozzle wall ${ }^{5,17}$ 


\subsubsection{Bottom Plate}

The rocket was designed with an aluminum bottom plate that extends to the end of the nozzle. The total length of the aluminum piece is 4 inches. Beyond the end of the nozzle, a piece of 0.75 inch glass forms the bottom of the mixing duct in order to accommodate the Schlieren and shadowgraph flow visualization systems. Figure 3-4 shows the bottom plate with the nozzle side wall.

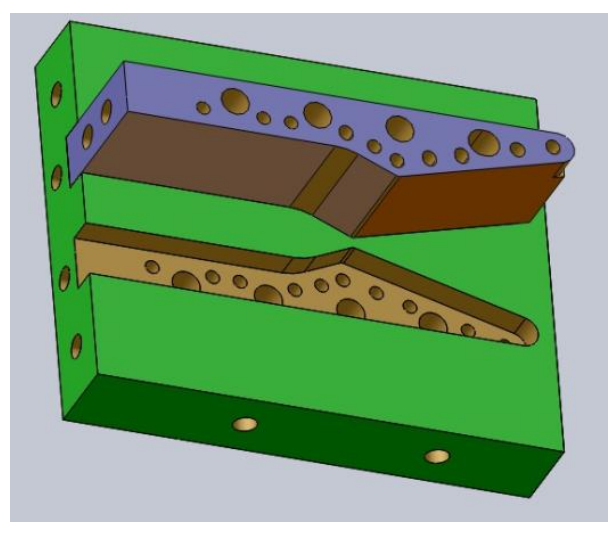

Figure 3-4: Bottom plate with nozzle wall ${ }^{5,17}$

\subsubsection{Side Walls}

The side walls were reconstructed in order to increase the length of the mixing duct. The purpose for increasing the length was to ensure that back pressure from the exit of the rocket was not affecting flow properties in the region of the mixing duct directly behind the nozzle. The length of these side walls was determined based on a suggestion by Addy that the length be 9 times the diameter of the mixing duct ${ }^{8}$. Since our duct is not axisymmetric the hydraulic diameter of the duct was used for this calculation. The right side has a static and stagnation port in the secondary duct, used to determine secondary flow conditions. The left side has static ports in the secondary duct and along the wall in the mixing duct. 


\subsubsection{Glass and End Caps}

The glass used to construct the top and bottom sections of the mixing duct was three quarter inch thick fused silica glass. Fused silica was used because it is able to withstand thermal loading better than ordinary glass. After looking into acquiring the glass it was found that a length of more than 14 inches would be very expensive. As a result we were forced to complete the upper and lower wall with aluminum end caps. The fused silica began to crack over time, and eventually fractured, making it unusable. The new glass, used for the final set of tests was Plexiglas. The Plexiglas was cut to fit the entire length of the mixing duct, without end caps. Figure 3-5 shows an assembly of the side wall, bottom plate, glass plate and nozzle wall.

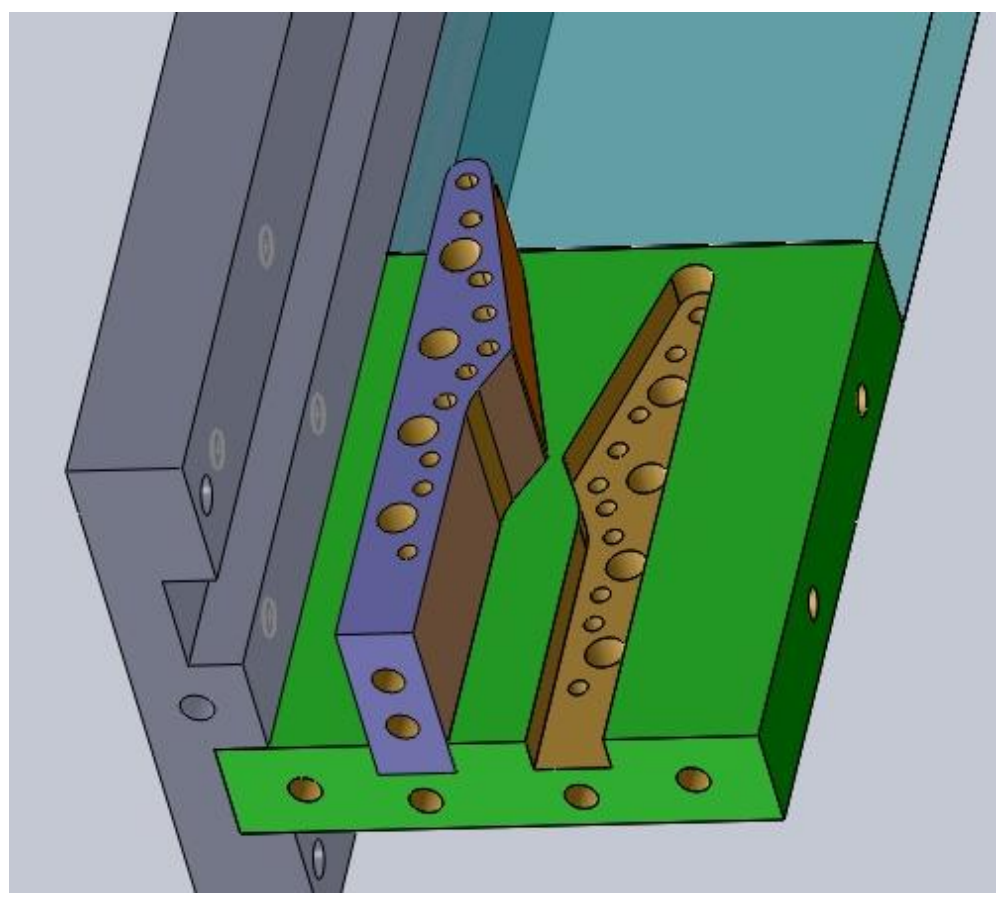

Figure 3-5: Assembly of bottom plate, nozzle wall, side wall and glass ${ }^{5,17}$ 


\subsubsection{Manifold}

The manifold of the rocket connects the incoming primary feed line to the chamber of the rocket. It also has channels which support the flow of secondary air to the rocket. The channels are constructed so that they have a constant area that is equal to the secondary area at the nozzle exit plane. Figure 3-6 shows a front view of the manifold.

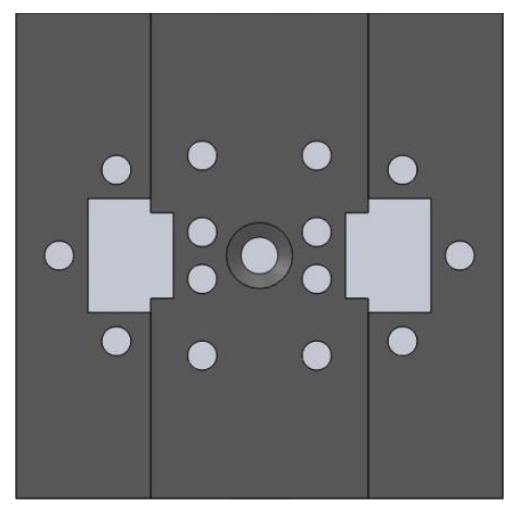

Figure 3-6: Front view of rocket manifold ${ }^{5,17}$

\subsection{Feed System}

The secondary ducts of the rocket are connected to a plenum chamber, allowing for lower ambient pressures. The plenum allows testing to achieve higher pressure ratios as well as simulate high altitude conditions. The plenum chamber is connected to the secondary ducts via a line of square tubing.

The rocket runs using a direct connect feed system, where high pressure nitrogen is fed into the rocket chamber through a series of feed lines and valves. One drawback of the set up was that our facilities do not have a regulator that can handle the high pressures and mass flow rates required by the experiments. As a result, none of the data is steady state because the chamber pressure is constantly changing. The feed system is "powered" 
by bottles of compressed nitrogen. These bottles have a pressure of $2600 \mathrm{psi}$, and are used to fill up a buffer tank. The buffer tank has a pressure transducer hooked up to it in order to identify its operating pressure. The buffer tank is also fitted with one inch diameter feed lines exiting it in order to help reduce line loss. The flow exits the buffer tank, and then passes through a ball valve. The valve is actuated by a Sharpe ${ }^{\circledR}$ SPN-063 "spring return" valve actuator which is powered by compressed air. In order to open the valve, it must be connected to a 100psi source as well as a power source. During testing, the valve was controlled via its electrical connection. After the valve, the flow continues through the one inch feed lines then is reduced to a 0.75 inch feed line before reaching the chamber. The pressure is then recorded again in the chamber. Figure 3-7 shows a diagram of the feed system.

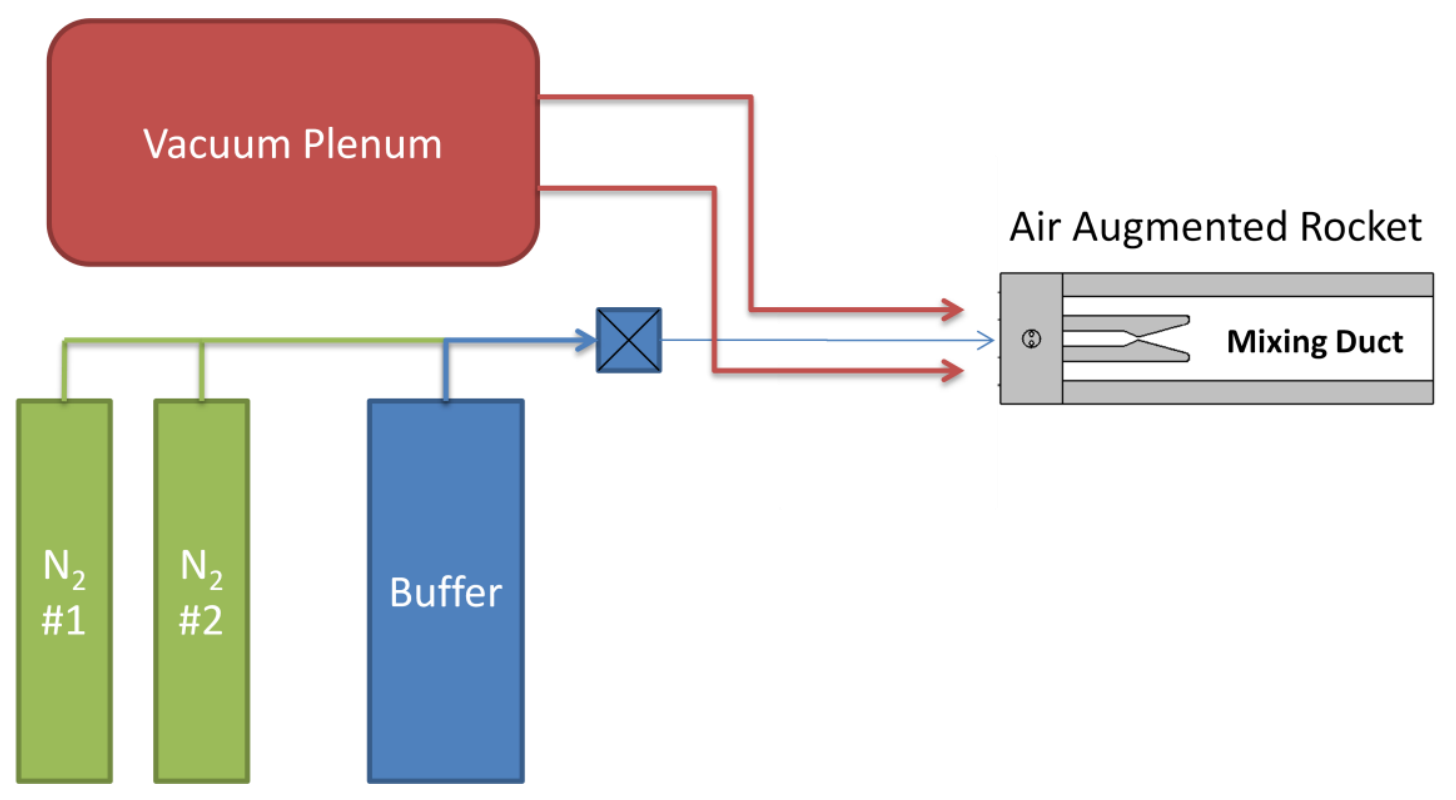

Figure 3-7: Feed system diagram 


\subsection{Plenum Chamber}

The plenum chamber was constructed using a reinforced oil drum, which was reinforced using welded bar stock. The reinforced oil drum is capable of pulling a vacuum down to 4 psi. Figure 3-8 shows the interior construction of the plenum chamber.

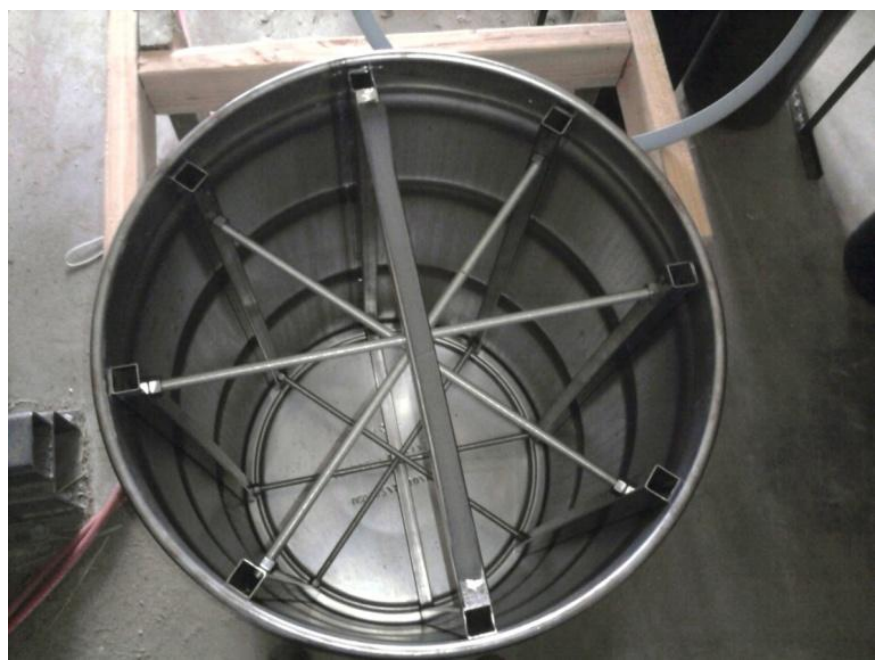

Figure 3-8: Interior of plenum chamber

Several different valves and gauges were machined into the top plate of the oil drum. There is an analogue gauge and a pressure transducer attached to the drum in order to measure its pressure. In addition, there is a manually operated relief valve connected to the plenum. When the pressure in the plenum reaches 4 psi, a switch is flipped and the relief valve is opened, insuring that the plenum does not implode during testing.

While constructing the plenum, a number of different methods were used to connect the plenum to the manifold of the rocket. Initially, a length of flexible tubing was used to connect the outlet of the plenum to the inlet of the manifold. The tubing created several issues in the feed system. The first problem was that considerable line losses in the hose caused a large amount of pressure loss, and the reduced area of the hose 
prevented choking from occurring in the mixing duct. Also, there were several abrupt area changes in the feed system causing the flow to be highly non-isentropic. These issues can have a significant effect on measurements taken in the flow field, and the subsequent calculation methods using those measurements. In order to correct the problem, the outlet of the plenum chamber was changed from flexible tubing to a solid stretch of square tubing. The square tubing connects directly into the manifold, reducing line losses and keeping a constant area from the plenum to the mixing duct. Figure 3-9 shows a picture of the plenum connected to the manifold by both the hose and the square tubing.

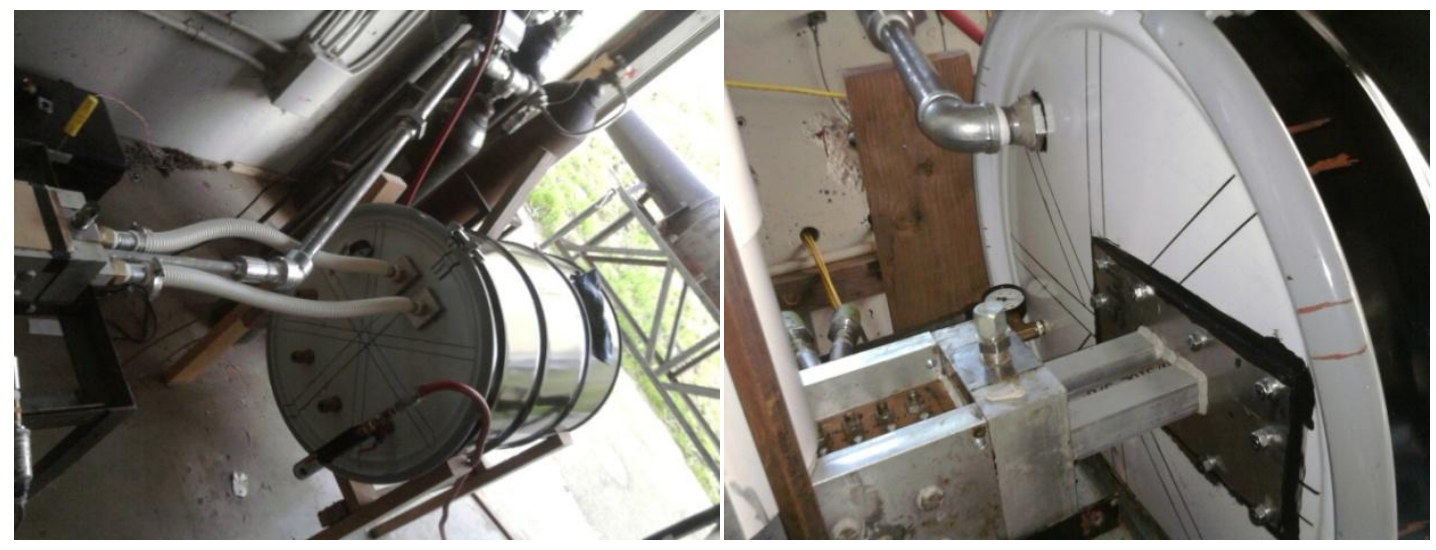

Figure 3-9: (left) Plenum connected to manifold with flexible tubing; (right) plenum connected to manifold with square tubing

\subsection{Inlet horns}

Inlet horns were created in order to ensure the flow transitions smoothly from the plenum chamber into the square inlet. These inlet horns were manufactured by Gist as part of the original work on the $\mathrm{AAR}^{5}$. The horns ensure that there is no significant contraction of the flow as it enters the duct leading to the manifold. The inlet horns are located inside the plenum chamber. They attach to the square tubing that runs into the 
manifold of the rocket. Figure 3-10 shows a picture of the inlet horns inside the plenum chamber.

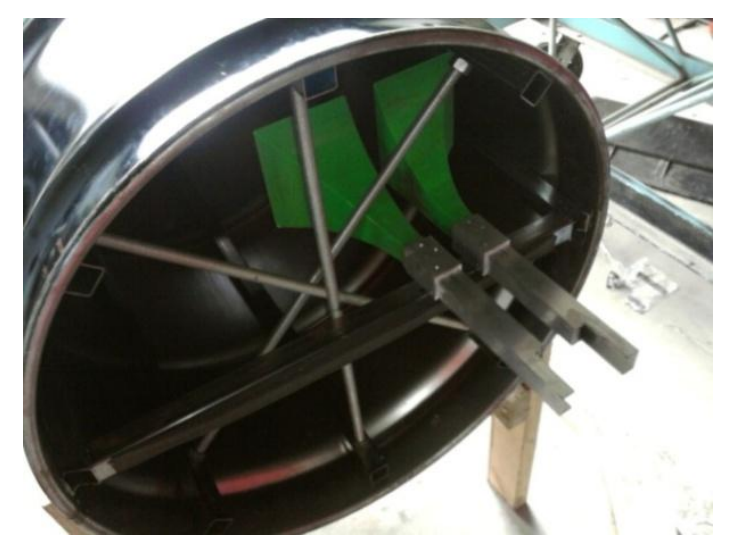

Figure 3-10: Inlet horns mounted inside of the plenum chamber 


\section{Data Collection}

A combination of pressure transducers, thermocouples and video images were used to collect data during the test runs. The following section gives a breakdown of how and where each of these devices were used, and how that data was processed.

\subsection{Pressure and Temperature Measurements}

Pressure transducers and thermocouples are used to measure the primary and secondary flow conditions. One static port is located in each of the inlet ducts and a stagnation port is located in the right inlet duct. The two static ports are used to compare the symmetry of the flow, while the stagnation port is used to calculate the mass flow rate in the secondary duct. Additional pressure transducers were placed in the chamber of the rocket and at the exit of the nozzle, as well as along the side walls of the mixing duct. The static ports on the side wall were not all in use for each test. There were not enough pressure transducers to take measurements at each location during a test. Thermocouples were placed in the chamber and the left duct in order to assist with mass flow rate and density calculations. Figure 4-1 shows a layout of the different measurement devices located within the rocket. 


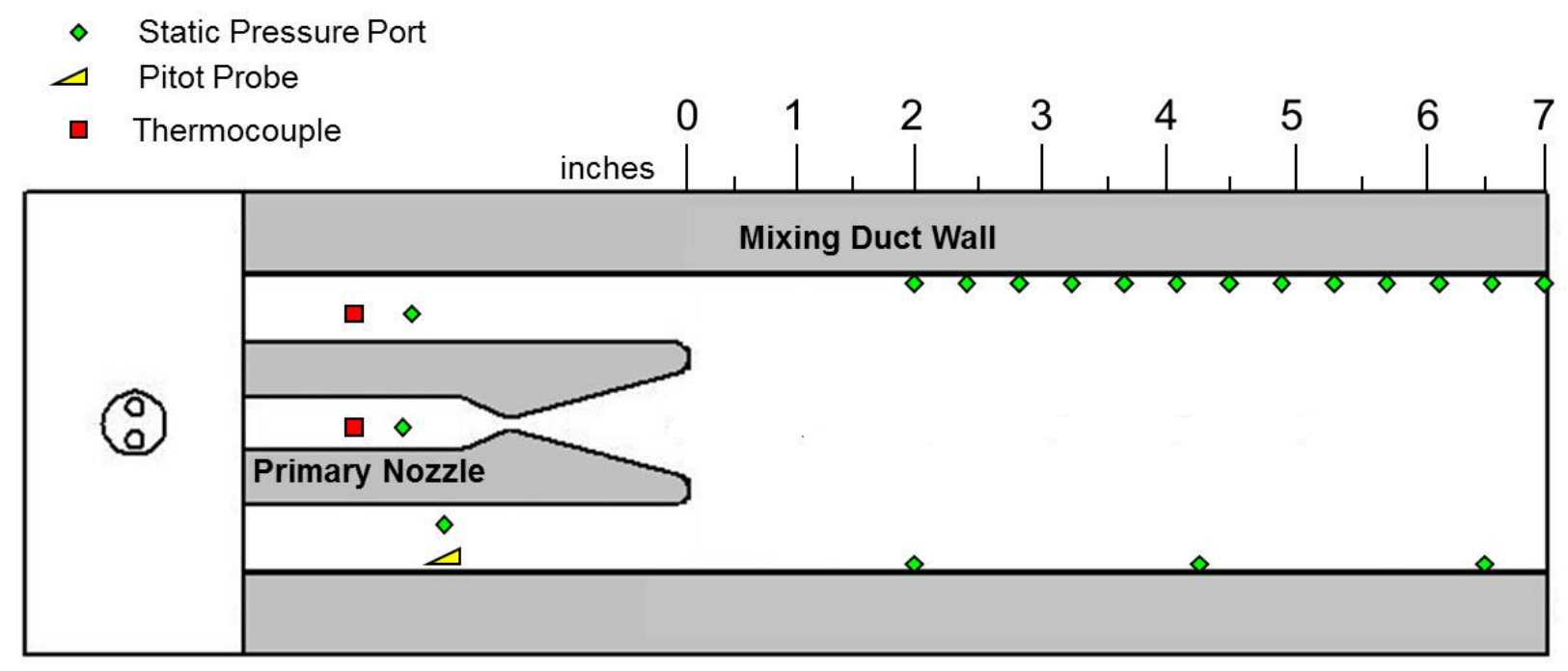

Figure 4-1: Layout of measuring devices on the rocket

The output from the pressure transducers are connected to a 32 channel National Instruments SCXI-1303 terminal block. The signal is then run to a laptop computer via a PCMCIA DAQ card-6036E, and calibrated and recorded using the computer software LabView. LabView was used to create calibration curves for all of the pressure transducers and thermocouples. All test data was output into an excel file for post processing. LabView was also used to create a user interface for the test runs.

\subsection{Shadowgraph Images}

The shadowgraph images were recorded using a Phantom v310 high speed camera. The high speed camera is capable of taking high definition pictures at a rate of 3,250 frames per second and is capable of exposure times as short as 1 microsecond. The video camera receives the light that has been reflected through the test section via the mirrors in the shadowgraph device. In order to ensure the highest quality photos the 
exposure time of each frame had to be set to balance image brightness with image clarity. Because the conditions in the test section are unsteady, the image being projected into the camera is constantly changing. A longer exposure time causes images to appear fuzzy. For these tests the exposure time was set to 300 microseconds. The exposure time could not be reduced any further, because the amount of light produced by the xenon lamp was not great enough to support shorter exposure times, while still producing a bright image. Figure 4-2 shows the location in the mixing duct where the shadowgraph images were being captured. The image extends from the exit of the nozzle to 5 inches downstream.

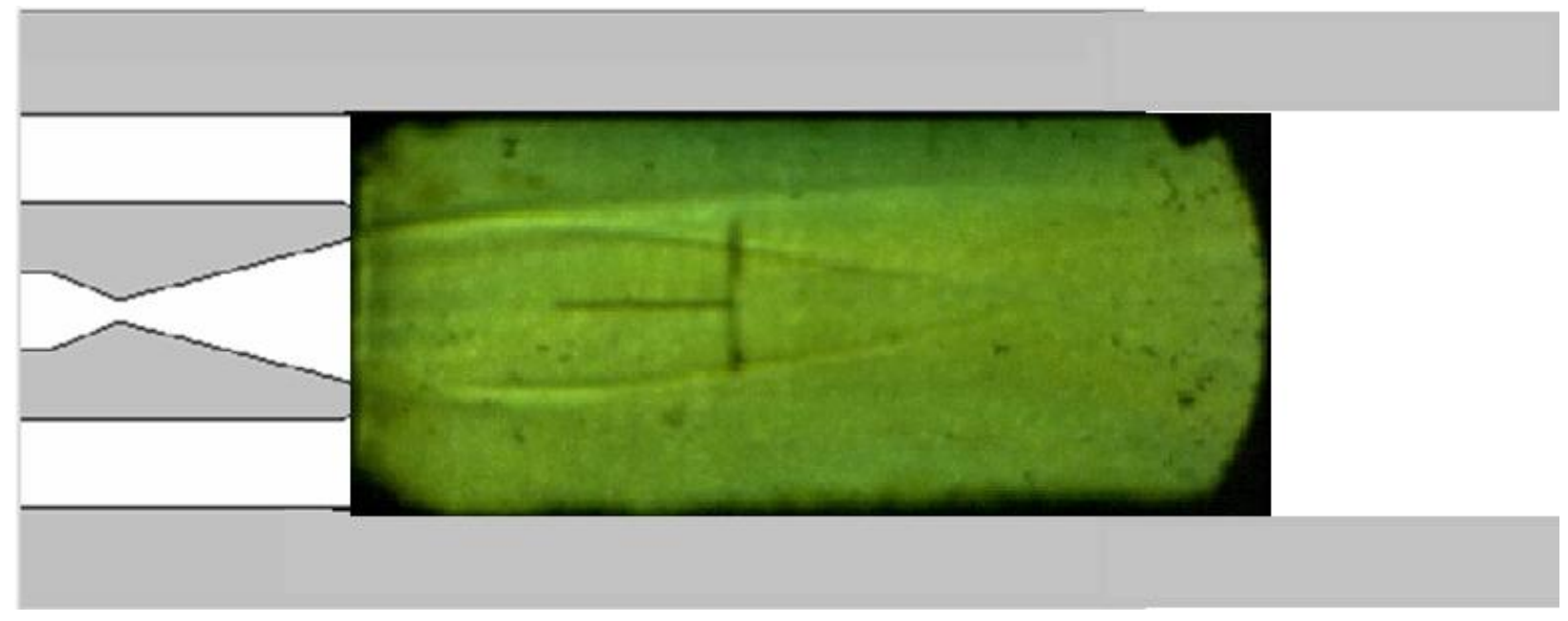

Figure 4-2: Location of Shadowgraph image within the mixing duct

Phantoms Cine viewer 675 was used in order to extract individual frames from the video. These frames represent still shots taken at specific times during the test. The time in the video recording was correlated to the time in the collected pressure transducer data in order to determine the pressure ratio being displayed in each picture. Once the pressure ratio for each picture was known they were analyzed using the program Digitize-it. The distance from the centerline of the duct to the side wall was measured in order to check the image for distortion. The distance between the centerline and the duct 
wall was found to be within $0.2 \%$ of the actual distance and the wall had a slope of less than 1 degree. This shows that the image is not being distorted and the results of further measurements can be trusted. 


\section{Theoretical Analysis}

The following section outlines the methods used to analyze the test results. A 2-D simulation was used to draw comparisons to the plume shape observed in the mixing duct of the AAR. The oblique shock prediction was produced using isentropic equations found in Gas Dynamics Volume $1^{19}$.

\subsection{CPSE Simulation}

In order to analyze the flow field within the mixing duct of the AAR, a 2-D inviscid code was written to approximate the interaction between the primary and secondary flows. The code was produced as part of a Master's thesis project by Brett Morham and will be referred to as the CPSE (Cal Poly Supersonic Ejector) simulation. The CPSE simulation uses Method of Characteristics to predict the shape of the primary plume. The secondary flow is approximated as 1-D, isentropic and reaching Mach 1 at its minimum area ${ }^{18}$. By assuming that the secondary flow is pressure matched to the boundary of the primary plume, the entire flow field can be predicted.

The Method of Characteristics uses information at two points in a flow to find the properties of a point downstream of the original two points. By marching downstream, the simulation can solve for conditions in a $2-\mathrm{D}$ supersonic flow ${ }^{18}$. In addition to calculating points within the plume, the simulation is also capable of calculating points along a fixed wall boundary and a free pressure boundary. 
Once the Method of Characteristics has been used to form an initial guess of the primary plume shape, isentropic relations can be applied to the secondary flow ${ }^{18}$. The plume boundary gives an available area for the secondary flow to pass through. The CPSE simulation assumes that the flow is choked at the point of minimum area. Using the choked flow assumption and the isentropic area ratio shown in Equation 5-1, the Mach number can be calculated in the secondary flow along the stream-wise direction.

$$
\frac{A_{s i}}{A_{s}^{*}}=\frac{1}{M_{s i}}\left(\frac{2}{\gamma+1}\right)\left(1+\frac{(\gamma-1) M_{s i}^{2}}{2}\right)
$$

In Equation 5-1 the subscript "s" refers to the secondary flow, the "i" means the conditions at a position in the mixing duct prior to the choke location, while the star represents conditions at the choke location.

Once the Mach number is determined at each point the static pressure at each stream wise location can be found using Equation 5-2.

$$
\frac{P}{p_{s i}}=\left(1+\frac{\gamma-1}{2} M_{s i}^{2}\right)^{\frac{\gamma}{\gamma-1}}
$$

Once the pressure distribution along the secondary flow has been solved, the primary plume can be recalculated using the new free pressure boundary. The entire process must be iterated until the solution is converged.

The idea for the scheme used in the CPSE simulation came from a doctorial thesis by $\mathrm{Addy}^{8}$. The inviscid solution can predict the shape of the primary plume as it interacts with the secondary flow in the mixing duct. An example of the results of the CPSE simulation is shown in Figure 5-1. Figure 5-1 shows the Mach number in primary and secondary flows. 


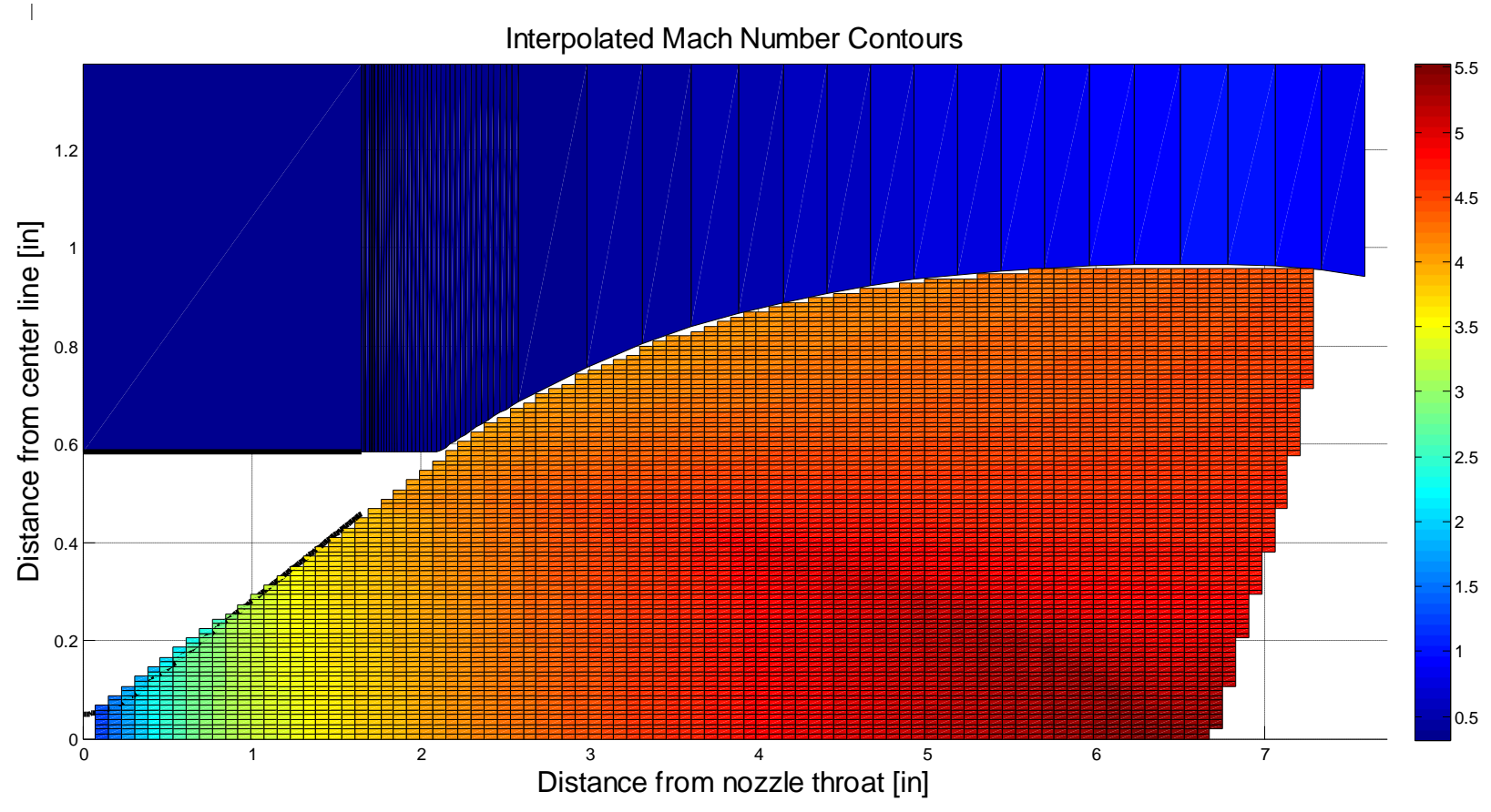

Figure 5-1: Inviscid code prediction of primary plume expansion ${ }^{18}$

The CPSE simulation was validated by comparing the entrainment ratios predicted by the simulation to the entrainment ratios measured experimentally, and calculated by a quasi 1-D analysis ${ }^{22}$. Figure 5-2 shows red circles representing the entrainment ratio predicted by the CPSE simulation. The solid lines are predictions of a quasi 1-D analysis completed by Gist. The blue dots are the entrainment ratios calculated from experimental data gathered by Gist. 


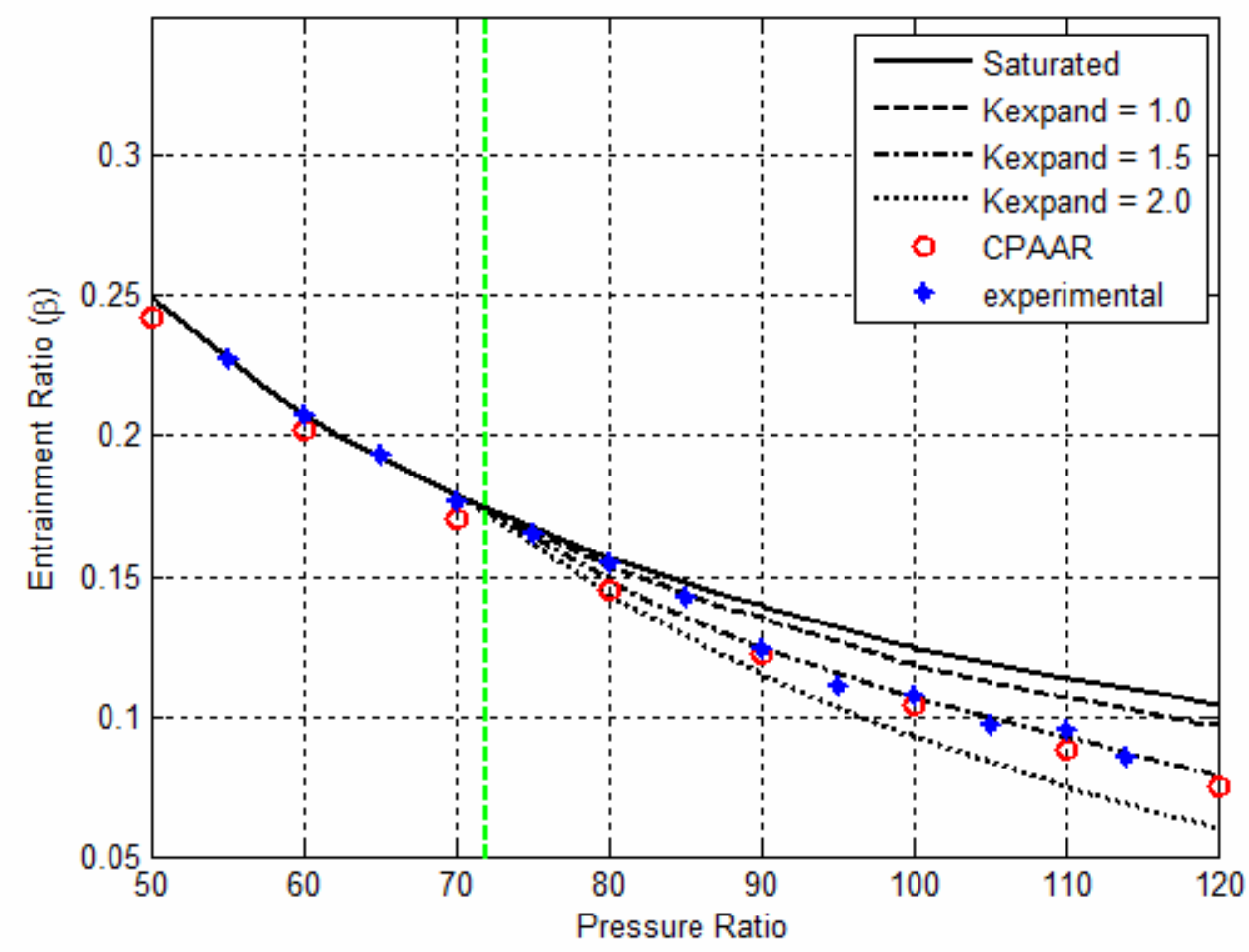

Figure 5-2: Validation of CPSE simulation ${ }^{18}$

Figure 5-2, above, shows a strong correlation between the results of the CPSE simulation and experimental data. The correlation gives confidence that the simulation is capable of predicting entrainment ratio.

\subsection{Oblique Shock Calculation}

The following section will outline the procedure for predicting the location and angle of an oblique shock in the primary plume of the AAR. The first step to calculating the location and angle of the shock is to characterize the condition of the primary flow, namely whether it is over-expanded or under-expanded. If the flow is under-expanded 
then the pressure at the exit of the primary nozzle is greater than the pressure of the secondary flow in the mixing duct. The primary flow will need to drop in pressure in order to match pressure with the secondary flow. The pressure drop is achieved by accelerating the flow through an expansion fan. When a supersonic flow passes through an expansion fan it increases in Mach number, lowering the static pressure of the flow. The expansion fan emanates from the nozzle lip then reflects back up off the symmetry plane. Figure 5-3 below illustrates this phenomenon.

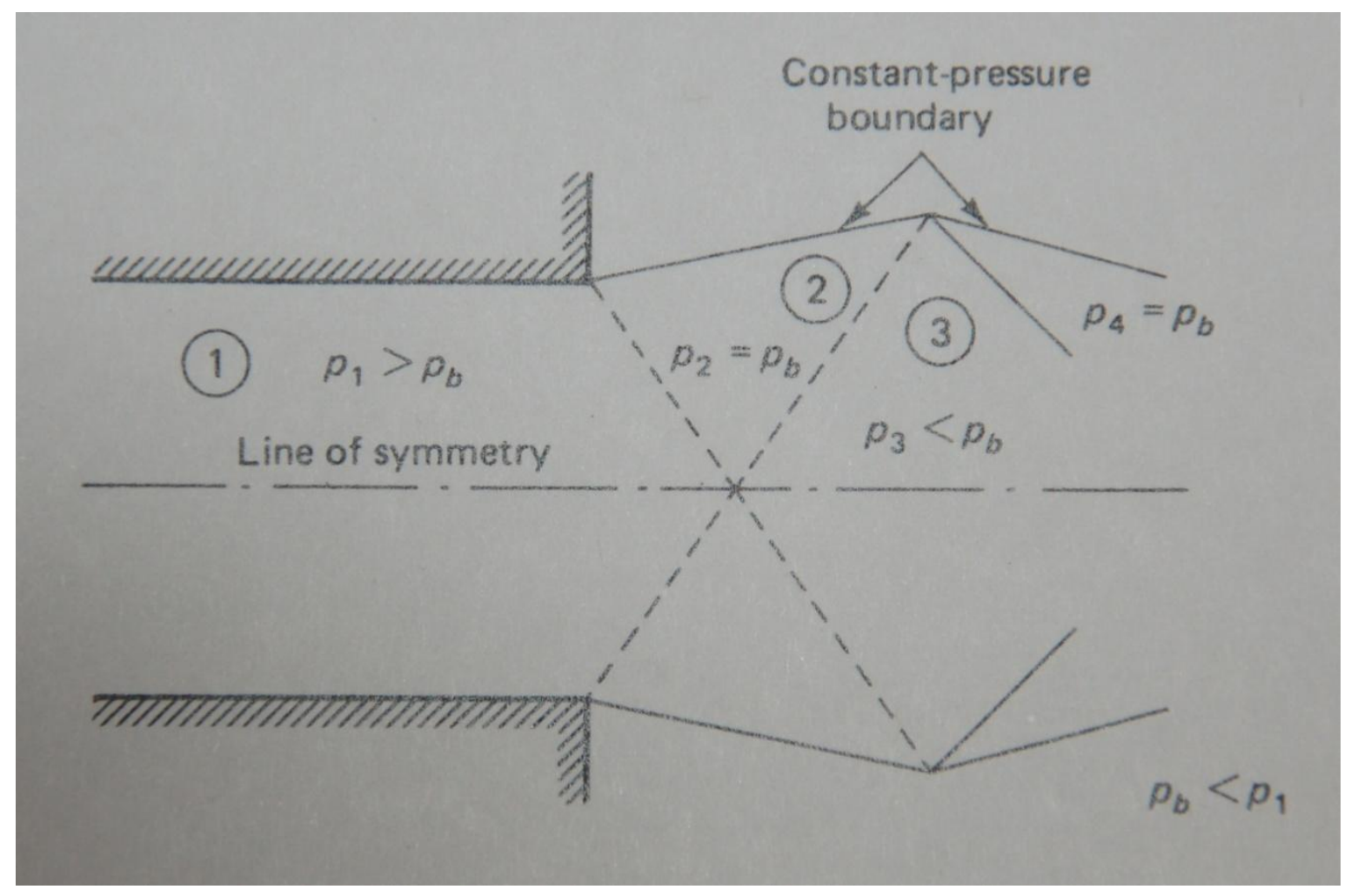

Figure 5-3: Under-expanded flow ${ }^{12}$

At this point it is helpful to break the flow field up into regions. Region 1 represents the primary flow conditions at the exit of the nozzle. Region 2 represents the primary flow at a condition where it is pressure matched with the secondary flow. A third region develops as a result of the expansion fan reflecting off the symmetry plane. 
In Region 3 the flow passes through the expansion fan and increases in Mach number, causing the static pressure in Region 3 to be less than the static pressure of the secondary flow. The pressure imbalance is corrected by the formation of an oblique shock. The oblique shock reduces the Mach number of the flow and increases the static pressure so that it is once again matched to the pressure in the secondary flow.

The first step in calculating the angle and location of the shock is to calculate the conditions at the nozzle exit and in the secondary duct. The primary flow Mach number at the exit of the nozzle can be calculated using the chamber pressure and static exit pressure of the primary flow. The static pressure in the secondary flow can be calculated using stagnation pressure of the secondary flow and by assuming that the secondary flow is choked. Equation 5-3 can be used for both of these calculations. For the secondary calculation the static pressure $(\mathrm{p})$ can be solved using stagnation pressure $(\mathrm{P})$ and Mach number (M). The equation can be rearranged to solve for Mach number using the static and stagnation pressures of the primary flow.

$$
\frac{P}{p}=\left(1+\frac{\gamma-1}{2} M^{2}\right)^{\frac{\gamma}{\gamma-1}}
$$

Once the static pressure of the secondary flow has been calculated, the flow can be classified as either over-expanded or under-expanded. If the flow is found to be under-expanded then the static pressure in Region 2 can be set equal to the static pressure of the secondary flow. Using the assumption of isentropic flow, the stagnation pressure in Region 2 is the same as the stagnation pressure at the nozzle exit. Equation 5-3 can now be used to calculate the Mach number in Region 2. 
The next step is to calculate the angle of the expansion fan. This calculation can be made using the Prandtl-Meyer angle. The Prandtl-Meyer angle represents the angle that a Mach 1 flow must be expanded around to reach a given final Mach number. Equation 5-4 shows how the Prandtl-Meyer angle can be calculated for a given Mach number. The Prandtl-Meyer angle is a function of Mach number and the ratio of specific heats of the fluid.

$$
v=-\sqrt{\frac{\gamma+1}{\gamma-1}} \tan ^{-1} \sqrt{\frac{\left(M^{2}-1\right)(\gamma-1)}{\gamma+1}}+\tan ^{-1} \sqrt{\left(M^{2}-1\right)}
$$

At this point the Prandtl-Meyer angle for the Mach number in Regions 1 and 2 can be solved. The difference between these two angles represents the deflection that the flow must pass through to accelerate from $\mathrm{M}_{1}$ to $\mathrm{M}_{2}$. The angles themselves can be plotted as originating from the nozzle lip and then reflecting off of the symmetry plane at the same angle. Figure 5-4 below shows an illustration of the behavior of the expansion fan. 


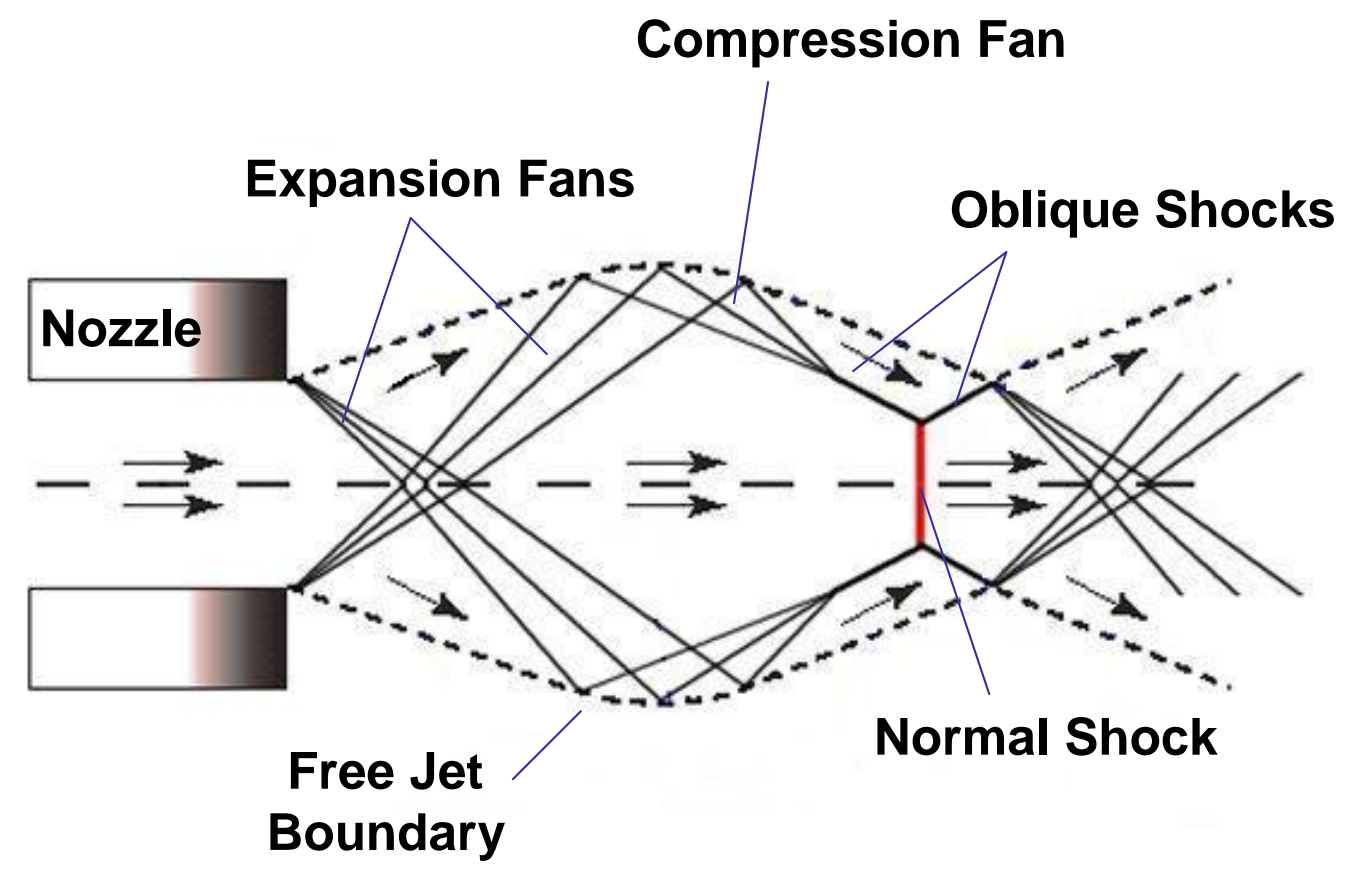

Figure 5-4: Expansion Cycle ${ }^{20}$

Figure 5-4 shows the expansion fan emanating from the lip of the nozzle. It then reflects off the symmetry plane before reaching the free jet boundary. The free jet boundary shows where the jet plume is pressure matched to the surrounding air. The expansion fan reflects off the free pressure boundary and coalesces into an oblique shock. The oblique shock forms inside the free pressure boundary and is not in contact with the boundary.

The next step in the analysis is to calculate the Mach number in Region 3, after the flow passes through the reflection of the expansion fan. In order to do this the Prandtl-Meyer angle for Region 3 must be calculated and then used to solve for the Mach number. Equation 5-5 shows how the calculation is made.

$$
\delta=v_{2}-v_{1} \quad v_{3}=v_{2}+\delta \quad(\text { Eq. } 5-5)^{19}
$$


The difference between the Prandtl-Meyer angles for Regions 1 and 2 equals the angle delta. This angle is added to the Prandtl-Meyer angle from Region 2 to get the Prandtl-Meyer angle for Region 3. Equation 5-4 can be solved numerically to output Mach number for a given Prandtl-Meyer angle. Now the Mach number for Region 3 is known. Using Equation 5-3 the static pressure in Region 3 can be solved. At this point the static pressure in Region 3 is less than the static pressure of the secondary flow. An oblique shock wave will form to correct this pressure difference. An illustration of this shock wave was shown in Figure 5-4.

The next step is to calculate the angle of the oblique shock wave. The angle can be calculated using the static pressures in Region 3 and the secondary flow. The pressure rise across the oblique shock can be equated to the pressure rise caused by a normal shock. The normal shock corresponding to that pressure rise will have an incoming Mach number that is less than the actual Mach number in Region 3. Equation 5-6 shows how this normal Mach number $\left(\mathrm{M}_{\mathrm{n}}\right)$ is calculated.

$$
\frac{p_{s}}{p_{3}}=\frac{2 \gamma}{\gamma+1} M_{n}^{2}-\frac{\gamma-1}{\gamma+1}
$$

The relationship between the actual and normal Mach number in Region 3 can be used to determine the angle of the oblique shock using Equation 5-7.

$$
\varepsilon=\sin ^{-1}\left(\frac{M_{n}}{M_{3}}\right)
$$

The variable $\varepsilon$ represents the angle of the oblique shock; however, it does not give any indication as to the location of the oblique shock. The location of the shock corresponds to the location where the expansion fan hits the boundary between the 
primary and secondary flows. In order to determine the location where the expansion fan meets the boundary the size of the primary plume must be determined. The Mach number in Region 3 can be used to calculate the cross sectional area of the primary plume. Equation 5-8 shows how the plume area is calculated.

$$
\frac{A_{3}}{A_{t}}=\frac{1}{M_{3}}\left[\frac{2}{\gamma+1}\left(1+\frac{\gamma-1}{2} M_{3}^{2}\right)\right]^{\frac{\gamma+1}{2(\gamma-1)}}+T
$$

The term $\mathrm{A}_{t}$ represents the throat area of the nozzle. The term $\mathrm{T}$, which is added onto the end of the equation, represents the thickness of the nozzle, which must be added to the area calculated from isentropic relations. Using the area calculated in Equation 5-8 the distance that the primary plume will expand from the centerline of the mixing duct can be determined. The expansion fan can now be plotted using the Prandtl-Meyer angle. Its reflection can also be plotted and will stop at the boundary of the primary plume, which was determined using Equation 5-8. Once the expansion fan reaches the boundary it will be reflected as an oblique shock. The angle of this shock was calculated using Equation 5-7. The shock will be projected out from the midpoint of the expansion fan as shown in Figure 5-5. Projecting that the oblique shock emanates from the midpoint of the expansion fan is an approximation. The oblique actually starts inside of the primary plume, where the expansion fans coalesce. 


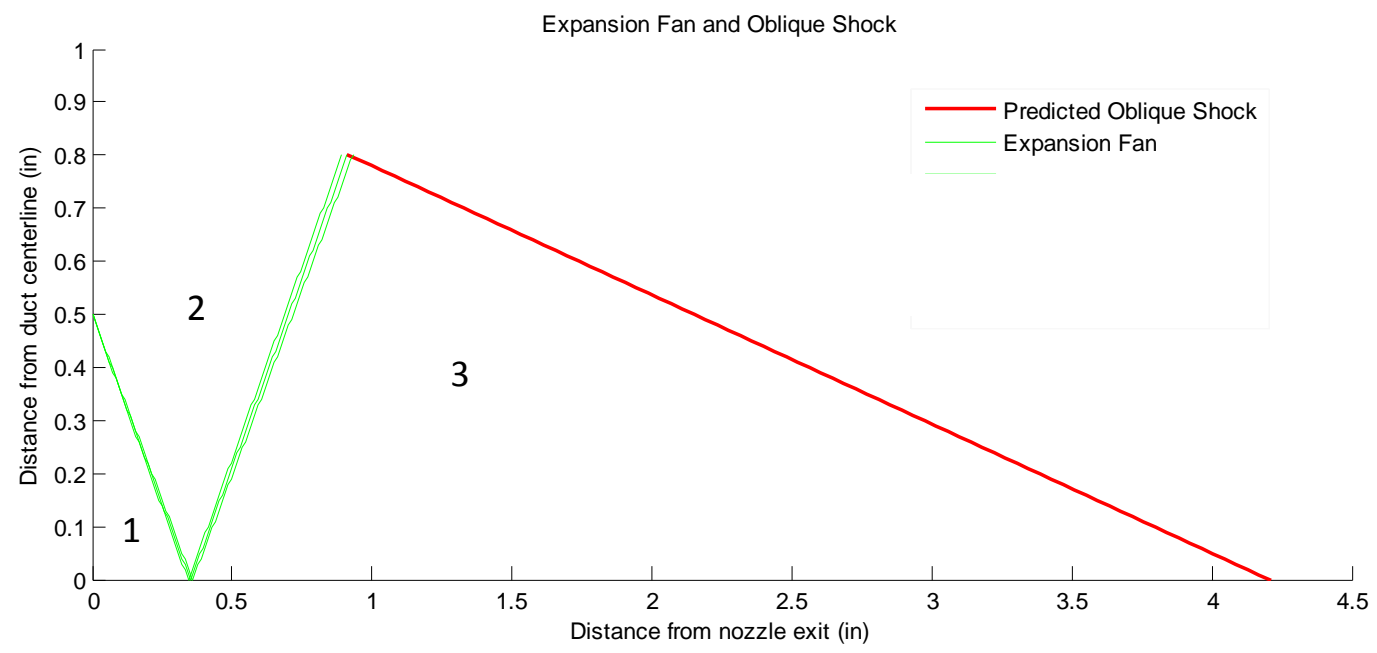

Figure 5-5: Expansion fan and oblique shock prediction

If the pressure at the nozzle exit is less than the pressure in the secondary flow then the primary flow is over-expanded and will not accelerate upon leaving the nozzle. Instead the flow will decelerate as it exits the nozzle in order to match pressure with the conditions in the secondary flow. An oblique shock wave will form, emanating from the nozzle lip, in order to slow down the primary flow. Figure 5-6 shows an illustration of what this looks like. 


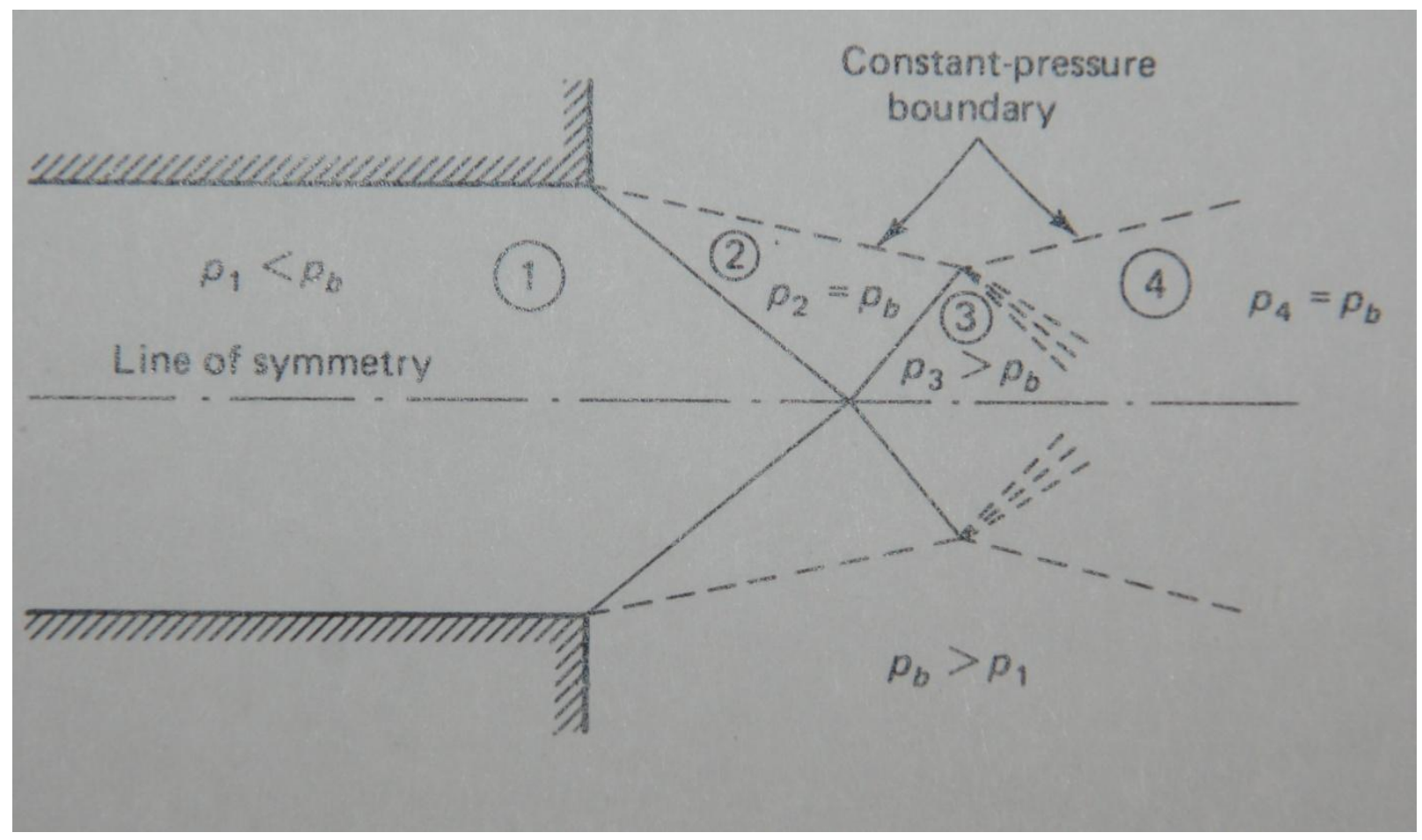

Figure 5-6: Over-expanded flow ${ }^{12}$

The over-expanded case allows for a simpler solution than the under-expanded case. There are no expansion fans to calculate, and the shock, which is the feature being calculated, forms at the lip of the nozzle. The only thing that must be calculated is the angle of the oblique shock. In order to determine the angle of the oblique shock the pressure at the exit of the nozzle will be compared to the pressure in the secondary duct. The pressure of the secondary flow cannot be calculated using the choked flow assumption when the flow is over-expanded. For the over-expanded case the measured pressure in mixing duct must be used in the calculation. Equations 5-6 and 5-7 can be used to determine the shock angle of the flow, using the pressure measured in the mixing duct during testing. 


\section{Error analysis}

There are two types of errors that must be dealt with when determining error in experimental data. These errors are systematic error and random error. The systematic error results from errors in calibration and inabilities to account for all environmental factors. Certain environmental factors such as turbulence in the flow can cause errors, and are difficult to account for. The systematic errors in our measurements were reduced through proper calibration of the measurement devises. Random error occurs from random fluctuations in the environment and in the measuring devise. Since these variations are random they will tend to occur about a mean value. In order to reduce random error each data point recorded by the pressure transducers is the average of 100 sample readings. This reduces the possibility of one bad reading by the pressure transducer causing a major error in calculations.

Some amount of random error in measurements is unavoidable and is the result of the finite precision of measuring devises. The pressure transducers used in this experiment have an accuracy of $0.25 \%{ }^{21}$. This creates an error in the readings which is then propagated through subsequent calculations using the pressure measurements. The error associated with each calculation was determined by taking the Pythagorean sum of each error source. Equation 6-1shows the formula used for combining error sources. In Equation 6-1 $\mathrm{Z}$ represents the variable that error is being calculated for, $\mathrm{F}$ is the function that solves for $\mathrm{Z}, \mathrm{A}$ and $\mathrm{B}$ are variables used to calculate $\mathrm{Z}$.

$$
\Delta Z=\sqrt{\left(\frac{\partial F}{\partial A}\right)^{2}(\Delta A)^{2}+\left(\frac{\partial F}{\partial B}\right)^{2}(\Delta B)^{2}}
$$


The total error in a calculation is a function of the error associated with each of the individual components. Below is a table giving error values based on measuring accuracy for several quantities that appear in this paper. Table 6-1 shows the error values associated with the pressure measurements taken during the tests.

Table 6-1: Percent error for key variables calculated using pressure measurements

\begin{tabular}{|l|l|l|}
\hline Variable & Average & Standard Deviation \\
\hline Secondary Mach Number (Ms) & $3.65 \%$ & $0.34 \%$ \\
\hline Mach Number Region 1 (M1) & $0.82 \%$ & $0.29 \%$ \\
\hline Mach Number Region 2 (M2) & $0.81 \%$ & $0.28 \%$ \\
\hline Mach Number Region 3 (M3) & $0.95 \%$ & $0.35 \%$ \\
\hline Prandtl-Meyer Number Region 1 (v1) & $0.19 \%$ & $0.004 \%$ \\
\hline Prandtl-Meyer Number Region 2 (v2) & $0.17 \%$ & $0.003 \%$ \\
\hline Epsilon ( $(\varepsilon)$ & $1.31 \%$ & $0.06 \%$ \\
\hline
\end{tabular}

There are several sources of error that are not related to measuring accuracy in the pressure transducers. These errors are associated with the data collected by the video camera as well as the data collected from the images. There is an error associated with correlating the frames from the video with the pressure and temperature data. There is also error associated with the points placed on the picture in order to determine the angle of the oblique shock. Both of these errors need to be addressed in order to determine the accuracy of the calculations.

The camera used for these tests was set at a frame rate of 300 frames per second. The pressure transducer and thermocouple data is collected at intervals of every 0.02 seconds. In order to correlate the pictures to the pressure and temperature data the start time of the test has to be found and matched. The match may be inaccurate because the frame falls between two data points in the pressure data. The error associated with 
matching the pictures to pressure data was calculated by determining the maximum bound the picture might be representing. The picture might be corresponding to one time step before or after the correlated time. The change in the value of each variable was found over this time, spanning 0.04 seconds, and its corresponding percent error is shown in the Table 6-2. The table also shows the total error, which is a combination of the error resulting from frame-data correlation and measuring accuracy.

Table 6-2: Percent error associated with frame-data correlation

\begin{tabular}{|l|l|l|l|}
\hline Variable & Average & Standard Deviation & Total Error \\
\hline Ms & $0.93 \%$ & $0.25 \%$ & $3.76 \%$ \\
\hline M1 & $1.05 \%$ & $0.12 \%$ & $1.33 \%$ \\
\hline M2 & $1.04 \%$ & $0.12 \%$ & $1.32 \%$ \\
\hline M3 & $1.13 \%$ & $0.17 \%$ & $1.48 \%$ \\
\hline v1 & $0.22 \%$ & $0.001 \%$ & $0.29 \%$ \\
\hline v2 & $0.18 \%$ & $0.002 \%$ & $0.25 \%$ \\
\hline$\varepsilon$ & $0.84 \%$ & $0.07 \%$ & $1.56 \%$ \\
\hline
\end{tabular}

The final error associated with the calculations in this paper has to do with the ability to repeat measurements. In order to measure the observed oblique shock angle, points must be placed on the image along the oblique shock; which requires a precision that is not repeatable. Since the process is not repeatable there is a random error introduced into the calculation. The average error associated with correctly placing these points was determined by repeatedly measuring the oblique shock on the same picture. A picture was measured 30 different times, and the standard deviation of the results was multiplied by two to determine the human error of this method. The calculation resulted in an error of $5.51 \%$ for $\varepsilon$, the wave angle of the oblique shock. It also resulted in an error of 0.024 inches for the $\mathrm{x}$ and $\mathrm{y}$ location of an individual point. 
All of these errors were then combined together in order to form the total error of the parameter $\varepsilon$, the oblique shock wave angle. The breakdown of each error source and the total error of the shock wave angle are shown in Table 6-3.

Table 6-3: Breakdown of error for oblique shock angle

\begin{tabular}{|l|l|}
\hline Variable & Average Error \\
\hline Measurement precision & $1.31 \%$ \\
\hline Frame-Data correlation & $0.84 \%$ \\
\hline Human error & $5.51 \%$ \\
\hline Total Error for $\boldsymbol{\varepsilon}$ & $\mathbf{5 . 7 2 \%}$ \\
\hline
\end{tabular}




\section{$7 \quad$ Results}

Experimental results were gathered from 10 separate tests, which recorded pressure, temperature and visual data of conditions inside the mixing duct of the Cal Poly AAR. These tests made use of a plenum chamber to reduce the stagnation pressure of the incoming secondary flow. The primary flow was powered by pressurized nitrogen. Both the primary and secondary flows were unsteady, with a stagnation pressure that was constantly dropping. Table 7-1 shows the key characteristics of the 10 tests. Included in Table $7-1$ is data about the pressures in the primary and secondary flows, as well as camera settings for the shadowgraph images. Test 52 had different camera settings than the other tests. After Test 52 it was determined that the faster frame rate produced more images then necessary for analysis, resulting in data files that were much larger than needed.

Table 7-1: Test Matrix

\begin{tabular}{|c|c|c|c|c|}
\hline Test & $\begin{array}{l}\text { Max Pressure } \\
\text { Ratio }\end{array}$ & $\begin{array}{l}\text { Max Chamber } \\
\text { Pressure (psi) }\end{array}$ & $\begin{array}{l}\text { Min Plenum } \\
\text { Pressure (psi) }\end{array}$ & $\begin{array}{l}\text { Camera Frame } \\
\text { Rate (fps) }\end{array}$ \\
\hline 52 & 123 & 1407 & 5.72 & 2000 \\
\hline 53 & 106 & 1205 & 5.98 & 300 \\
\hline 54 & 116 & 1401 & 5.78 & 300 \\
\hline 55 & 105 & 1229 & 5.99 & 300 \\
\hline 56 & 116 & 1407 & 5.53 & 300 \\
\hline 57 & 120 & 1447 & 5.6 & 300 \\
\hline 58 & 127 & 1524 & 5.54 & 300 \\
\hline 59 & 128 & 1494 & 5.41 & 300 \\
\hline 60 & 132 & 1492 & 5.55 & 300 \\
\hline 61 & 113 & 1348 & 5.91 & 300 \\
\hline
\end{tabular}




\section{7-1 Oblique Shock Comparison}

The shadowgraph images contain information about the flow field in the mixing duct of the Cal Poly AAR. The images show the different regions of the flow field. Figure 7-1 is a photo taken from Test 60 at a pressure ratio of 132; in it the different regions of the flow field are labeled.

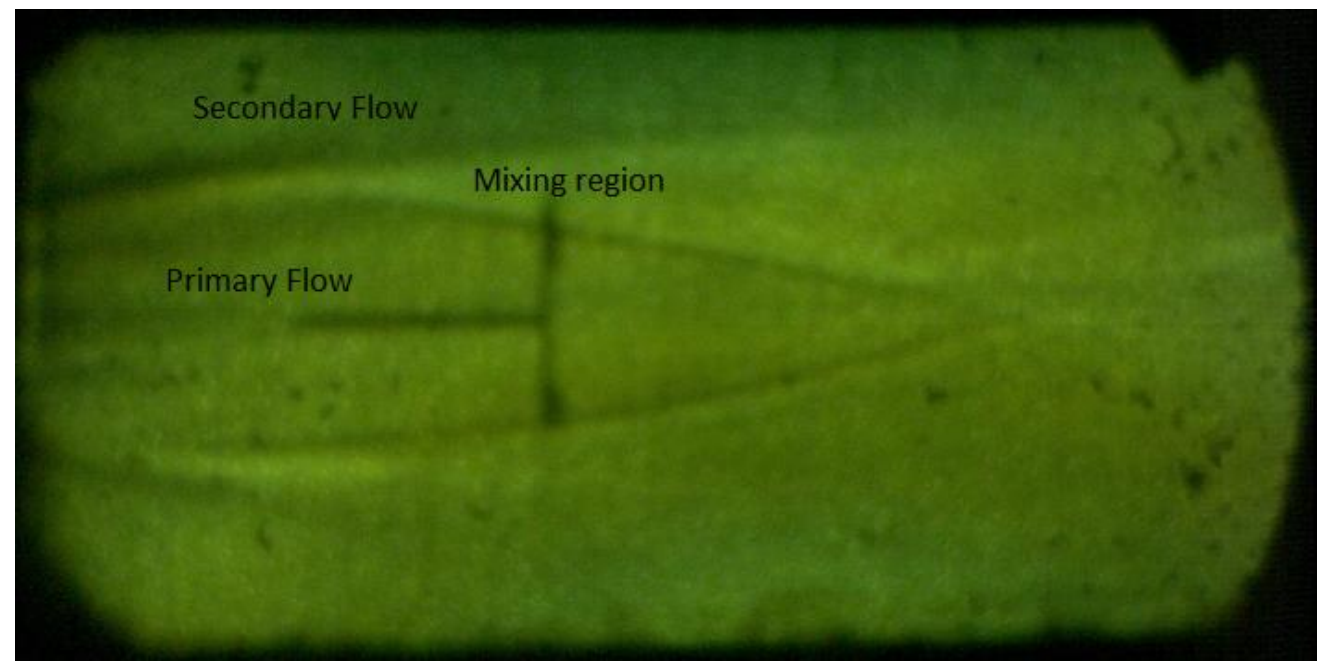

Figure 7-1: Flow field regions as seen in shadowgraph images

The secondary flow occupies the region closest to the wall and the primary flow occupies the center of the mixing duct. In between these regions is a third region where mixing is occurring between the primary and secondary flows. The mixing region is defined by the shadows that are cast along the edge of it. One thing to note is that the mixing region does not show any turbulent structures. This is due to the speed of the flow and the exposure time of the camera. The exposure time is 300 microseconds. In this time a particle in the secondary flow is traveling over half the length of the mixing duct shown in the image. A particle in the primary flow would pass across the entire picture in this time. Because the particles are moving the length of the picture during the 
exposure time it blurs out any turbulent structures. For this reason it is not possible to get a precise measurement of the turbulent region. The image does show the approximate size of the mixing region. At 2 inches downstream of the nozzle, marked by the vertical segment of the $\mathrm{T}$, the mixing region occupies approximately $20-25 \%$ of the available flow area. The image also shows a distinct transition between the mixing region and the primary flow. There is a very sharp line that transitions between the primary flow and the mixing region; this line is outlined in red in Figure 7-2, below.

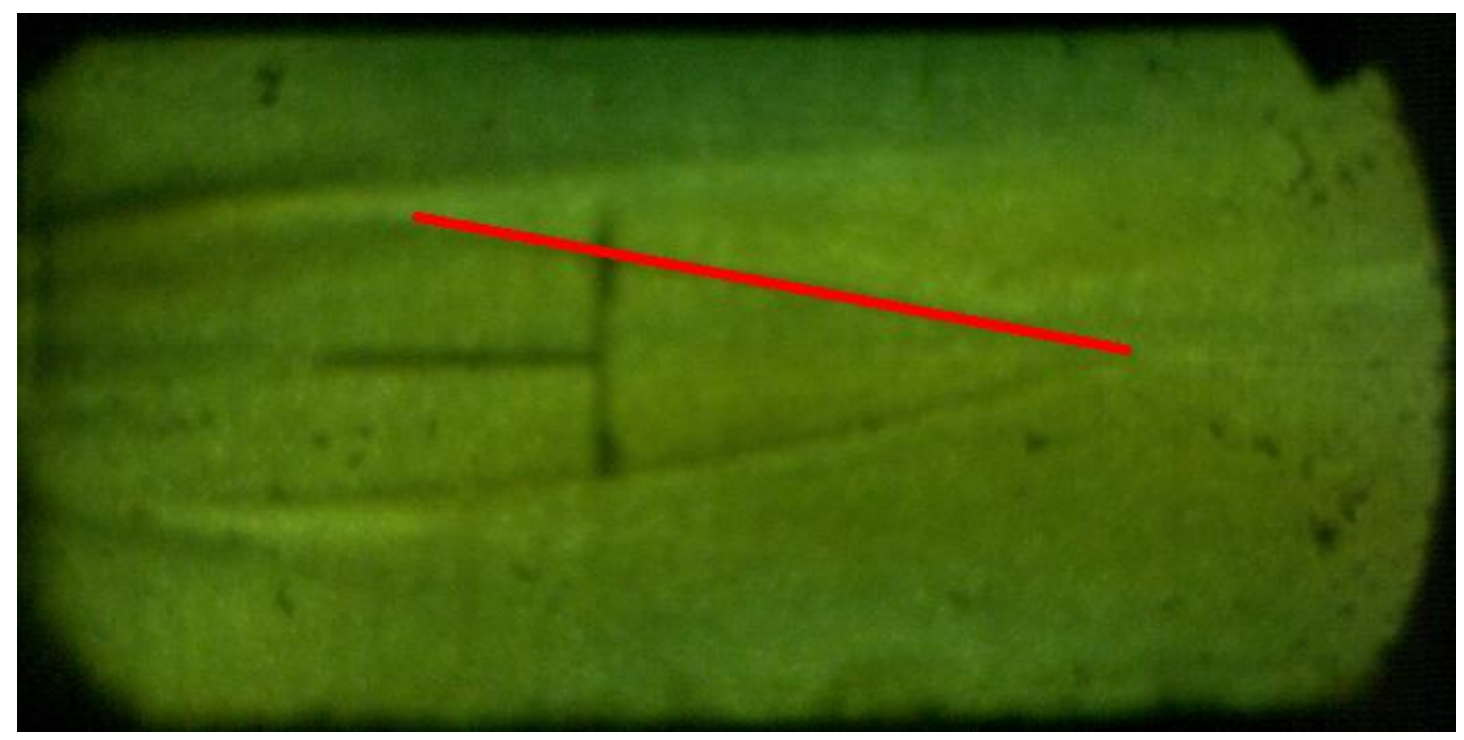

Figure 7-2: Oblique shock

The red line is believed to represent an oblique shock. Theory predicts that an oblique shock will form in the primary flow of an under-expanded supersonic exhaust as the result of an expansion cycle. After the primary flow expands out through expansion fans it is turned back in towards the centerline by the oblique shock. The angle and location of the shock can be measured against theoretical predictions to determine if what the image is showing is truly an oblique shock. 
The shadowgraph images contain information about the shape and angle of the oblique shock. The program Digitize-it was used to retrieve information from the images. Once the image was imported into Digitize-it, scale factors for the image had to be determined. Digitize-it records the distance between two points, but known distances need to be recorded in order to scale those readings. In order to assist in scaling the image, lines were drawn on the glass. At the center of the picture is a line that is 1 "long running down the center of the mixing duct with a 1 " line forming a right angle with the first line at 2" downstream from the nozzle exit. Figure 7-3 shows how these lines were used to scale the output from Digitize-it.

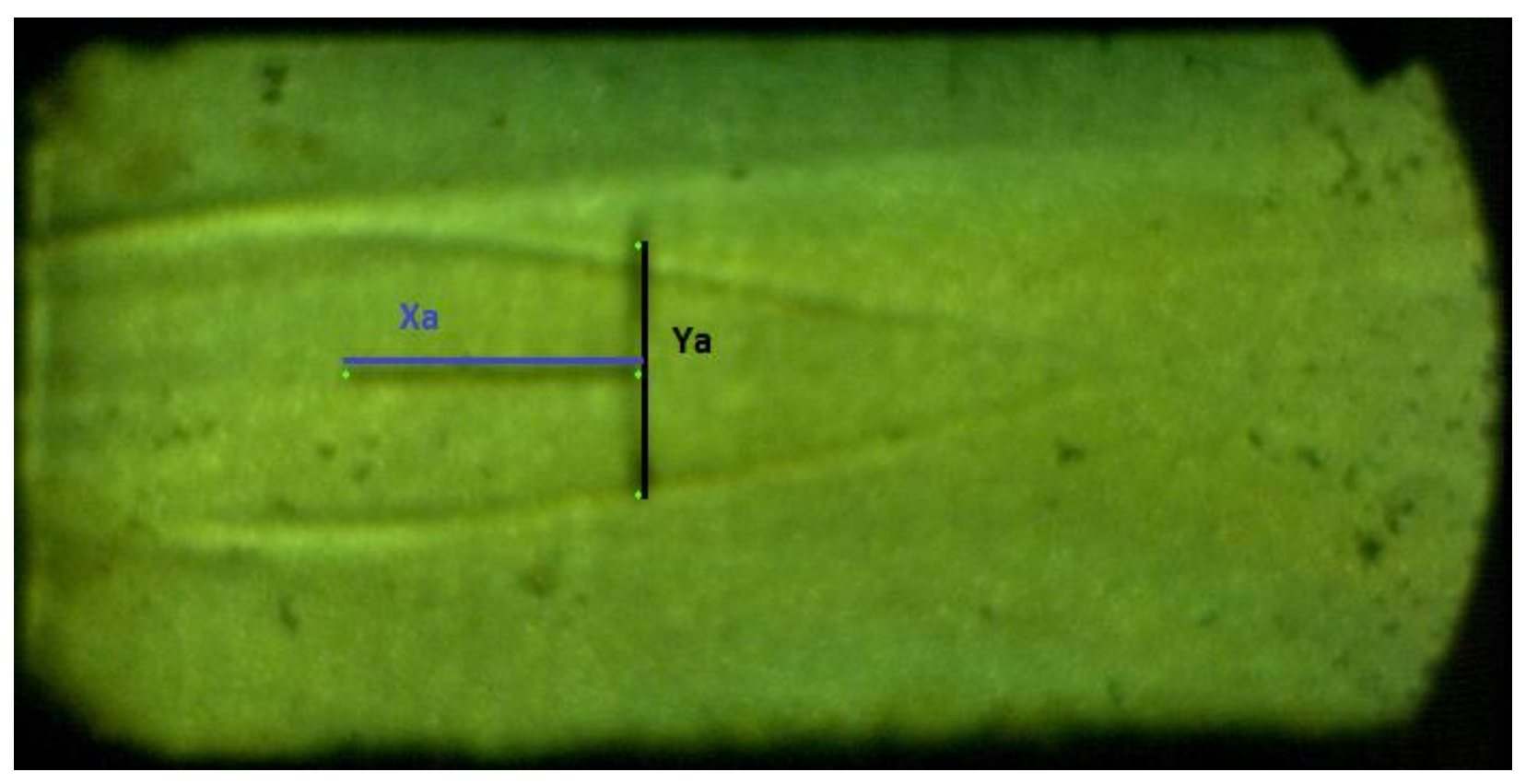

Figure 7-3: Points used for scaling image

The points used for scaling were placed at the ends of the two lines. The line Ya gives a scale factor for measurements taken in the $\mathrm{Y}$ axis. The line $\mathrm{Xa}$ gives a scale factor for the measurements taken in the $\mathrm{X}$ axis. The scale factor was calculated by dividing the actual distance Ya and Xa by the output given by Digitize-it. Once the scale 
factor had been determined, the oblique shock could be measured by placing points along the shock. The oblique shock was measured from the point it becomes clear, about 1.5 inches from the nozzle, until it reflects off the centerline of the mixing duct. Figure 7-4 shows the image with points placed along the shock.

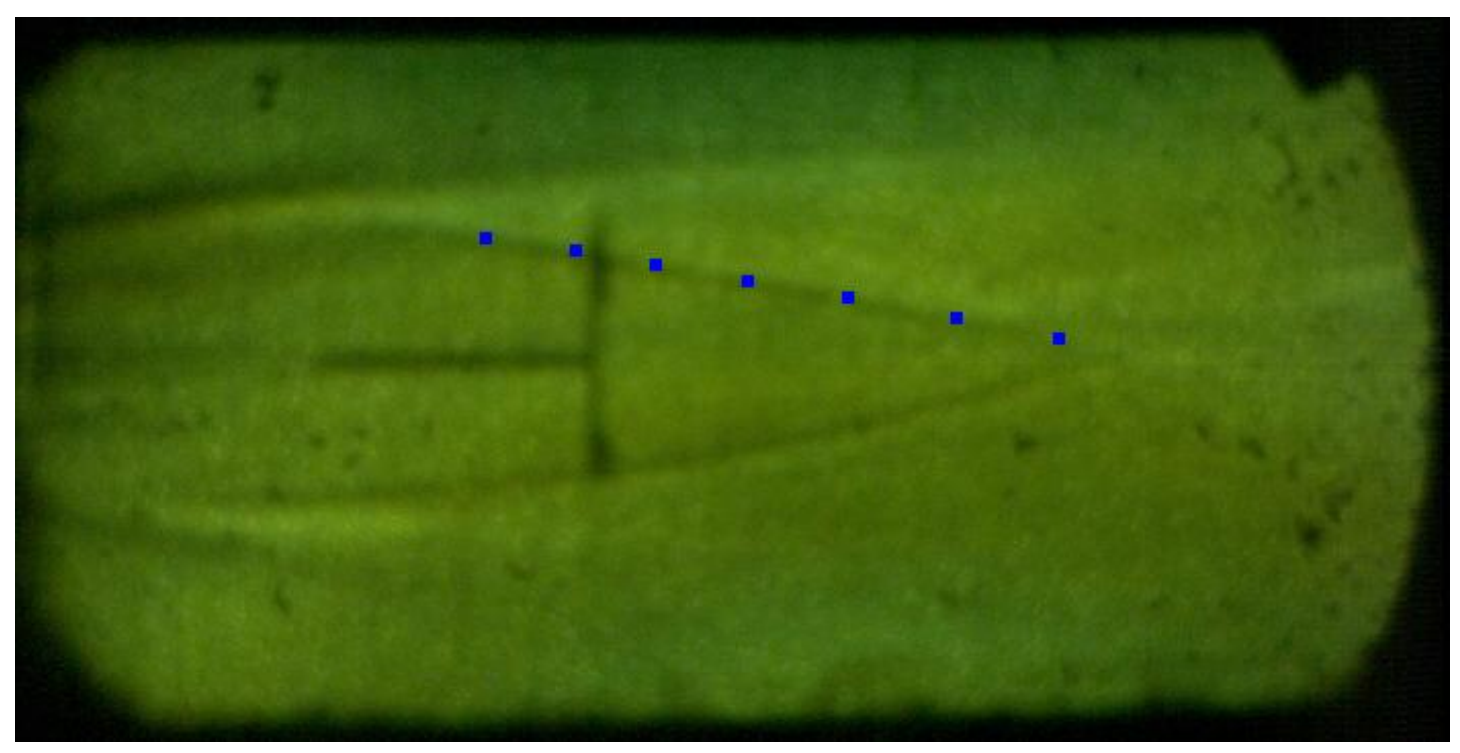

Figure 7-4: Oblique shock data points

The points outlining the oblique shock were imported into MATLAB in order to be scaled and processed. A linear best fit equation, produced using MATLAB's "polyfit" function, was used to determine the angle of the oblique shock. Figure 7-5 shows the points from the oblique shock imported into MATLAB and plotted with the best fit line of the oblique shock. 


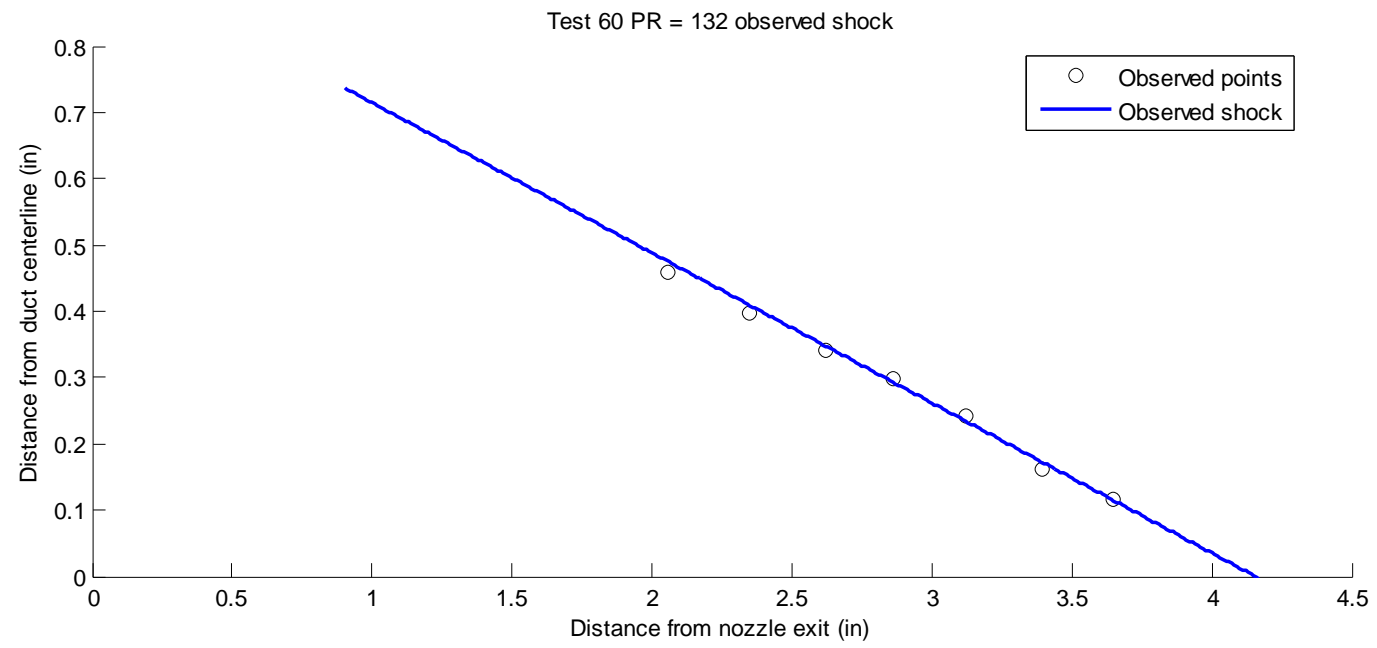

Figure 7-5: Oblique shock plotted in MATLAB

The oblique shock observed in the images and plotted in MATLAB can now be compared to the shock prediction that was outlined in the Section 5-2. Figure 7-6 shows the plot in Figure 7-5 with the prediction of the expansion fan and oblique shock. The regions of the flow field described in Section 5-2 are also labeled on Figure 7-6.

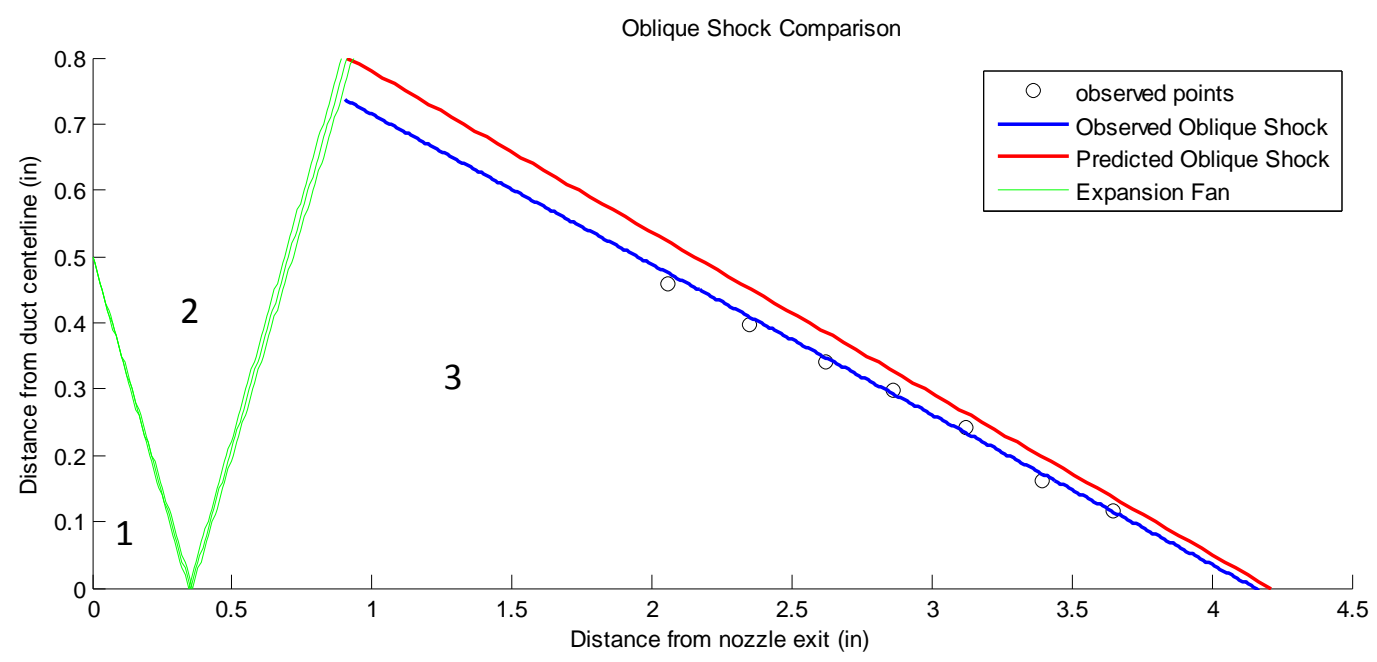

Figure 7-6: Test 60 PR = 132, oblique shock comparison 
The exact location of the shock is difficult to accurately predict. The point where the expansion fans merge into a shock is not well defined. At this point it may be helpful to refer back to Figure 5-4, which shows the expansion cycle in an under-expanded free jet. The expansion fan reflects off the free pressure boundary and then merges into an oblique shock at a point inside the free pressure boundary. In Figure 7-6 the shock is assumed to emanate from the midpoint of where the expansion fan would reflect off the free pressure boundary. This assumption gives an approximation as to where the shock is located but it is not an accurate reflection of what is occurring in the primary plume.

The location of the shock is even harder to predict when the flow is overexpanded. Theory assumes that an over-expanded flow will have a shock emanating from the nozzle lip. However, by looking at the oblique shock location for an overexpanded case in Test 60, we find that the shock does not start at the nozzle lip. For each test it was found that the flow transitions from under-expanded to over-expand at a pressure ratio of 100. A more detailed explanation of this transition is found in section 73. Figure 7-7 shows the location of the shock observed at a pressure ratio of 89 .

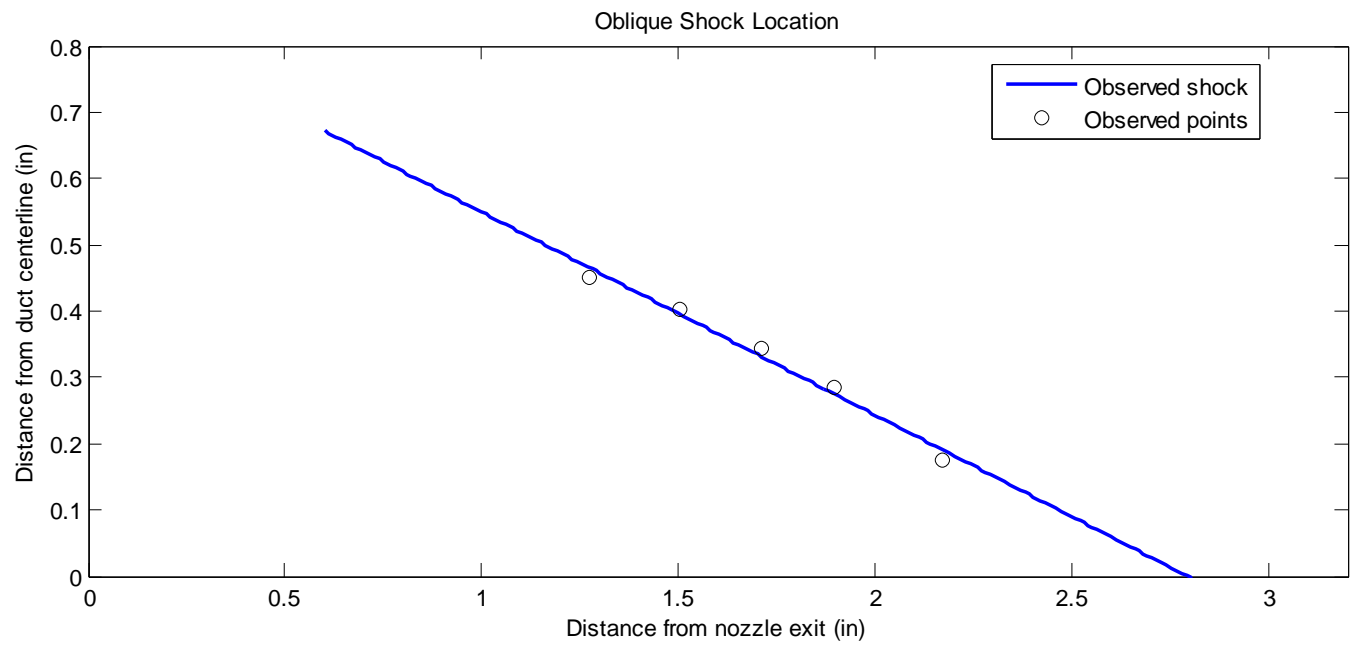

Figure 7-7: Test 60, $P R=89$ oblique shock location 
The oblique shock emanates from a point downstream of the nozzle. The edge of the nozzle lip is located at the point $(0,0.5)$. The reason that the shock occurs further downstream than predicted is most likely due to the effects of a recirculation zone. The recirculation zone that forms right behind the nozzle lip has a lower pressure than the secondary flow. It is not until the primary flow meets the secondary flow at the end of the recirculation zone that the oblique shock forms.

The angle of the shock can be calculated with more certainty and confidence. The shock wave angle is a function of measured pressures and is not dependent on the location of the expansion fans, or the size of the recirculation zone. Below, in Table 7-2, is a comparison of calculated and observed shock wave angles for Test 60 . The same comparison is shown for the other nine tests in Appendix A.

Table 7-2: Comparison of Oblique shock wave angles for Test 60

\begin{tabular}{|r|r|r|r|}
\hline Pressure Ratio & $\begin{array}{l}\text { Observed Angle } \\
(\mathrm{deg})\end{array}$ & \multicolumn{2}{|l|}{$\begin{array}{l}\text { Calculated Angle } \\
(\mathrm{deg})\end{array}$} \\
\hline 85 & 23.04 & 14.88 & 35.41 \\
\hline 96 & 15.31 & 14.48 & 5.42 \\
\hline 105 & 15.13 & 13.71 & 9.39 \\
\hline 113 & 15.61 & 13.56 & 13.11 \\
\hline 118 & 17.07 & 13.67 & 19.45 \\
\hline 124 & 16.91 & 13.62 & 0.08 \\
\hline 130 & 13.66 & 13.67 & 3.29 \\
\hline 132 & 13.19 & 13.63 & 0.52 \\
\hline 131 & 13.93 & 13.86 & 2.74 \\
\hline 126 & 13.40 & 13.77 & 9.60 \\
\hline 121 & 15.27 & 13.80 & 4.41 \\
\hline 115 & 14.31 & 13.68 & 12.49 \\
\hline 110 & 15.91 & 13.92 & 12.91 \\
\hline 105 & 12.58 & 14.20 & 4.49 \\
\hline 95 & 17.60 & 16.81 & 1.16 \\
\hline 89 & 18.11 & 18.32 & 1.96 \\
\hline 85 & 18.82 & 18.45 & 1.38 \\
\hline 78 & 18.92 & 19.18 & \\
\hline
\end{tabular}


The average difference between the observed and the calculated angles is $8.7 \%$ for all values. If the values taken during the most unsteady parts of the test, at the very beginning, are removed from the data set then the average difference becomes $4.6 \%$. The stretch of data from a pressure ratio of 130 down to 115 represents the best match between observed and calculated values. That range of pressure ratios also represents the quasi-steady portion of the test where the secondary Mach number only changes by $6.1 \%$. Since the steadier portion of the test produces a more favorable comparison it leads to the conclusion that some of the more inaccurate comparisons might be due to the unsteady nature of the tests.

Table 7-3 shows the average difference between calculated and observed shock wave angles for each test, along with the maximum pressure ratio of each test. In addition the table shows the standard deviation of the average and the adjusted average. The adjusted average shows the average difference with all values greater than one standard deviation away from the mean removed. This is done to limit the effect of outliers.

Table 7-3: Comparison of calculated and observed shock wave angles for Tests 52-61

\begin{tabular}{|l|l|l|l|l|}
\hline Test & Max PR & $\begin{array}{l}\text { Average \% } \\
\text { difference of } \\
\text { shock angle }\end{array}$ & $\begin{array}{l}\text { Standard } \\
\text { Deviation }\end{array}$ & $\begin{array}{l}\text { Adjusted \% } \\
\text { difference of } \\
\text { shock angle }\end{array}$ \\
\hline 52 & 123 & 17.0 & 9.7 & 12.9 \\
\hline 53 & 106 & 12.9 & 9.3 & 8.2 \\
\hline 54 & 116 & 10.8 & 4.7 & 9.6 \\
\hline 55 & 105 & 9.3 & 8.1 & 6.0 \\
\hline 56 & 116 & 11.5 & 6.4 & 7.7 \\
\hline 57 & 120 & 8.7 & 7.7 & 6.3 \\
\hline 58 & 127 & 9.5 & 6.2 & 7.2 \\
\hline 59 & 128 & 13.6 & 7.4 & 11.1 \\
\hline 60 & 132 & 8.7 & 9.1 & 5.3 \\
\hline 61 & 113 & 12.4 & 8.9 & 9.8 \\
\hline
\end{tabular}


The average difference for all the tests is $11.44 \%$ while the adjusted average is $8.41 \%$. With the exceptions of Tests 52 and 59 all of the tests were less than $10 \%$ in the adjusted difference. Some deviation between the predicted and observed values is to be expected. The predictions are based on isentropic flow assumptions. Real flow is never completely isentropic and will always have some discrepancy with isentropic calculations. The error in the calculation of the shock angle is $5.72 \%$. This error is an underestimate because it does not take into account the effect of the isentropic assumption or the effect of turbulence on the measuring devises.

The next comparison that can be made with this data is between the shock location and the wall pressure data. Data was taken along the wall of the secondary duct. As the flow moves downstream the pressure drops. At a certain point the pressure along the wall goes back up. Plotting the wall pressure distribution next to the shock location gives insight into what is causing the pressure drop and subsequent rise. Figure 7-8 shows the pressure distribution along the wall for Test 60 at a pressure ratio of 132 . Below the wall pressure plot is the plot of the observed oblique shock for Test 60 at a pressure ratio of 132 . 

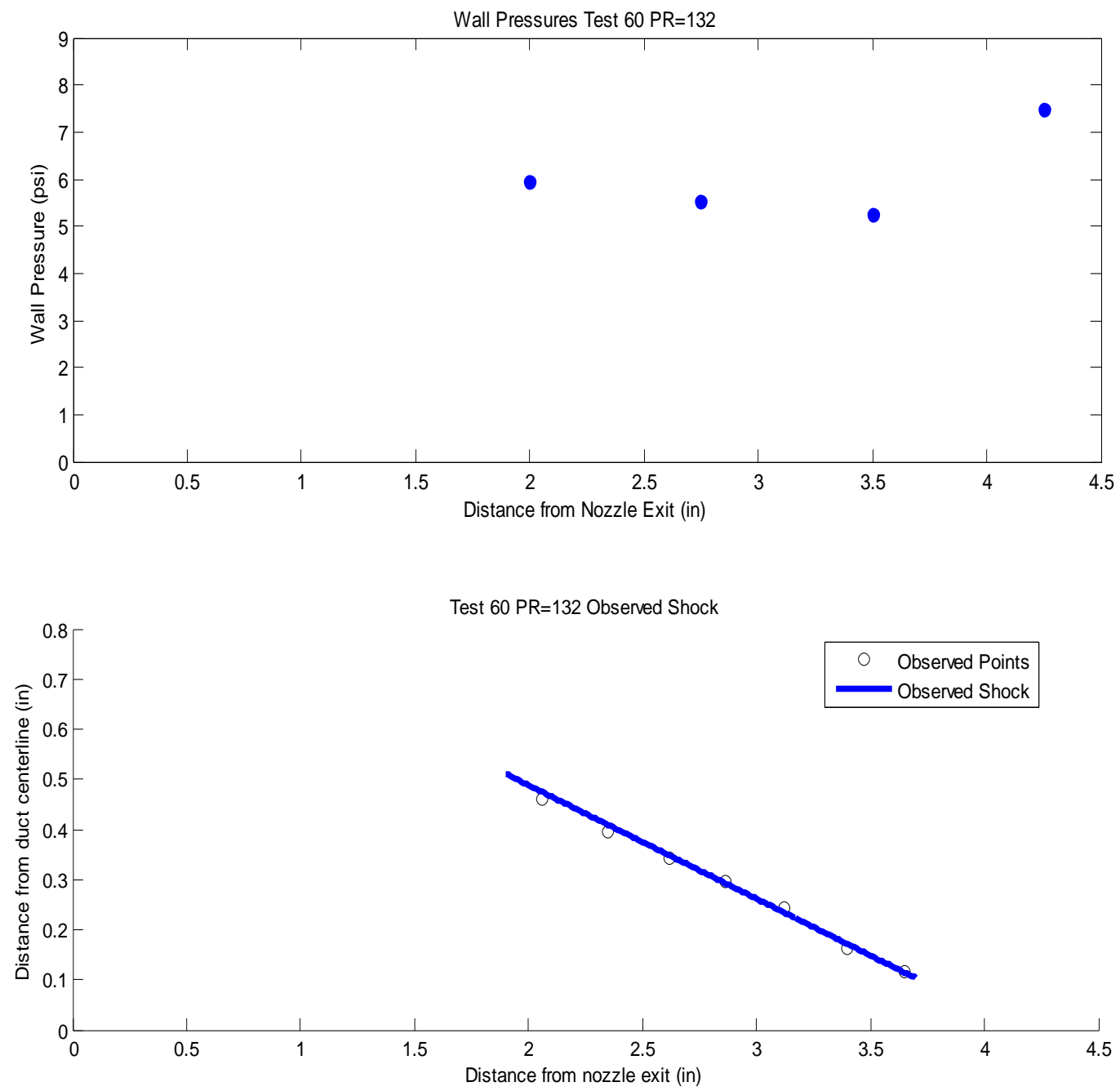

Figure 7-8: Wall pressure for Test $60 \mathrm{PR}=132$ compared to oblique shock observation

The comparison shows that the minimum wall pressure occurs at the same location that the oblique shock reaches the centerline of the mixing duct. The pressure then rises sharply in the region after the shock reflects off the symmetry line at the center of the mixing duct. This correlation can be observed at different pressure ratios, which reflect different minimum pressure locations. Figure 7-9 shows the wall pressures and shock location for Test 60 at a pressure ratio of 89 . 

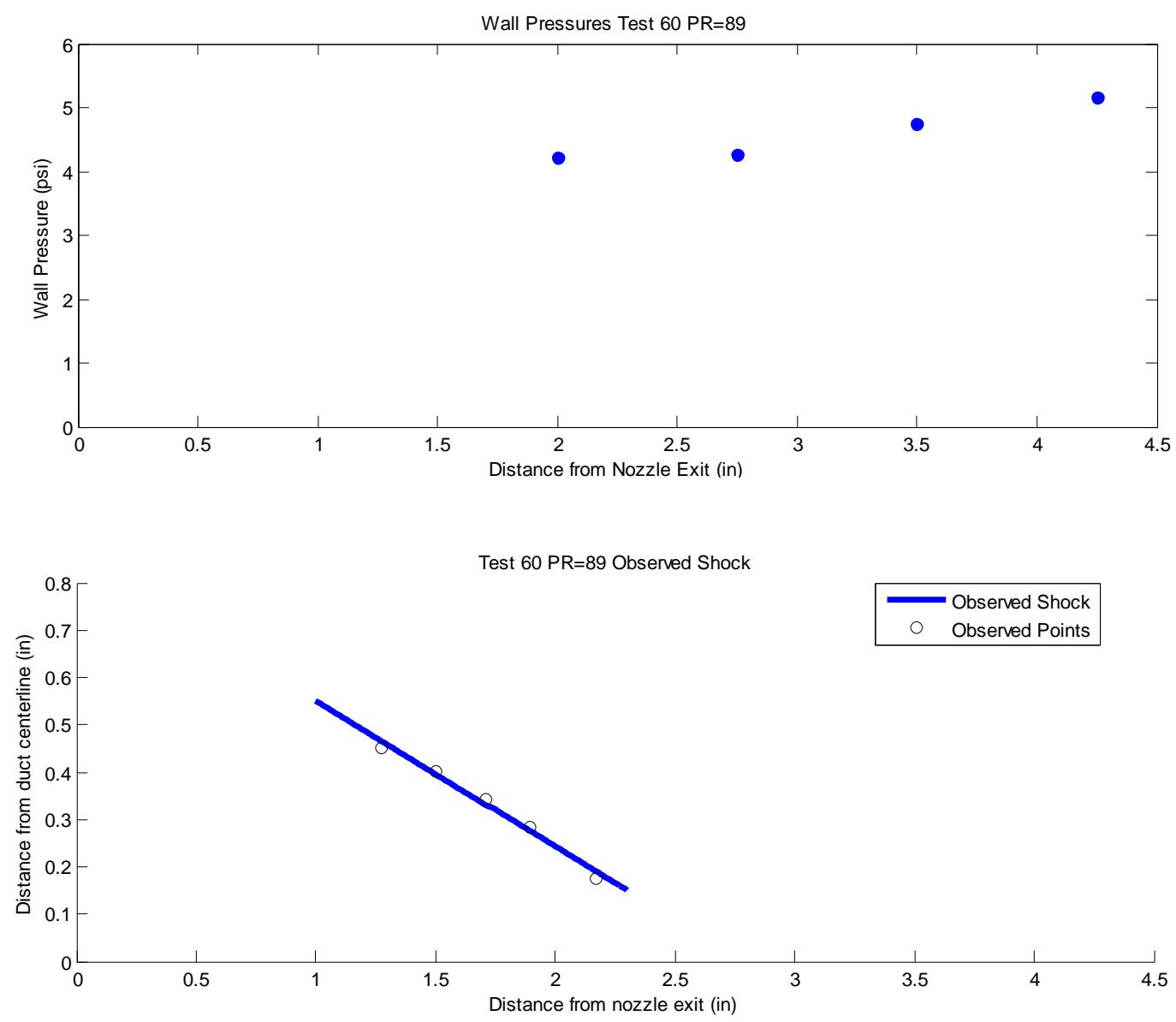

Figure 7-9: Test $60 \mathrm{PR}=89$, comparison of wall pressures and shock location

Once again the minimum pressure region corresponds to the location where the shock reaches the symmetry line at the center of the mixing duct.

After the shock reaches the symmetry plane it reflects off as an oblique shock. Figure 7-10 shows this reflection in an image taken from Test 60 at a pressure ratio of 132. 


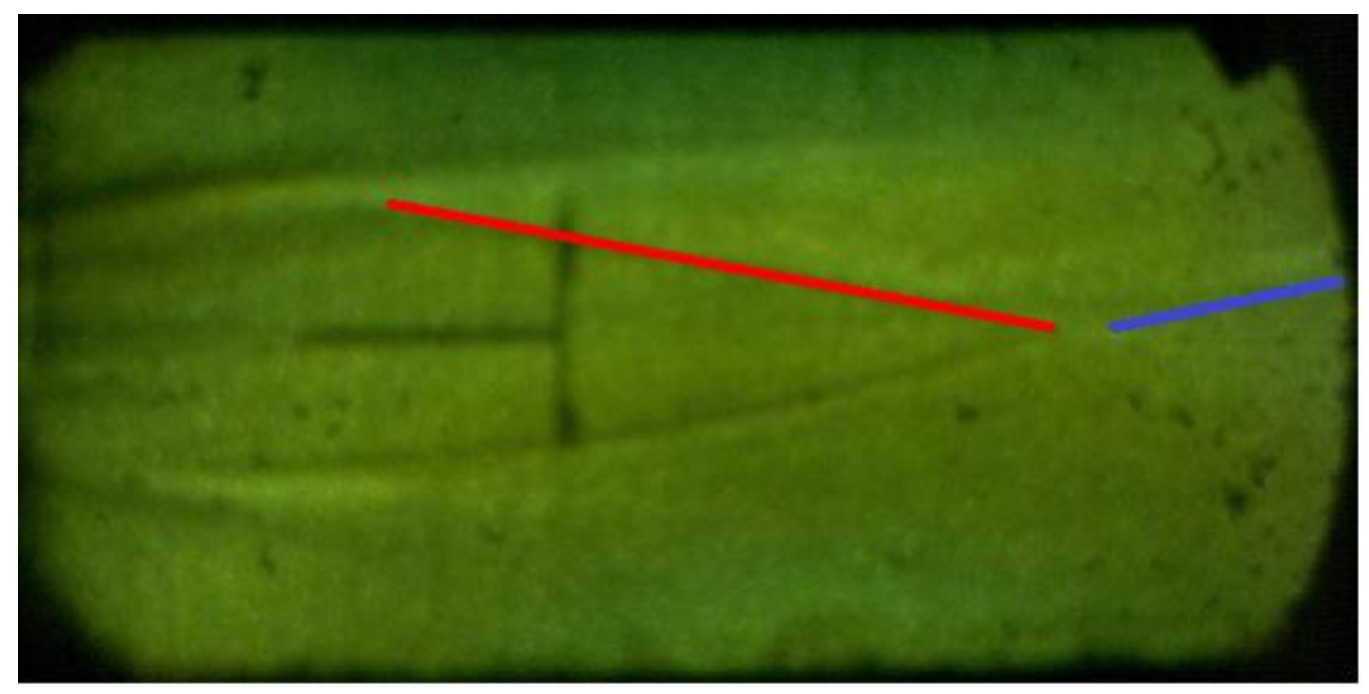

Figure 7-10: Oblique shock reflection off symmetry plane

The red line in Figure 7-10 represents the oblique shock analyzed previously, while the blue line represents the reflected shock. The reflected shock has an observed angle of 17.2 degrees; compared to the observed angle of 13.2 degrees for the original shock. A study of shock reflections from symmetry planes conducted by Hornung showed that weak shocks produce a reflection that is close in angle to the incoming shock $^{22}$. The study also showed that as the strength of the shock increased the angle of the shock steepened as it reached the symmetry plane, causing a Mach disc ${ }^{22}$. The images produced during our tests show that the shock forming in the primary flow is a weak shock, which does not increase in angle as it reaches the symmetry plane, and reflects regularly from it at an angle similar to its incident angle. 


\subsection{CPSE Simulation Comparison and Fabri Choking}

The shape of the mixing region was compared to the shape of the primary plume predicted by the CPSE simulation in order to attempt to validate the accuracy of the CPSE simulation. Below is a plot which overlays the observed mixing region and the plume boundary as predicted by the simulation. The plume boundary in the Figure 7-11 represents the edge of the observed mixing region. The line representing the simulation is the inviscid boundary predicted by the CPSE simulation. The y-axis is measured from the center of the mixing duct. Figure 7-11 shows the predicted plume shape and the actual plume shape during Test 60 at a pressure ratio of 132 .

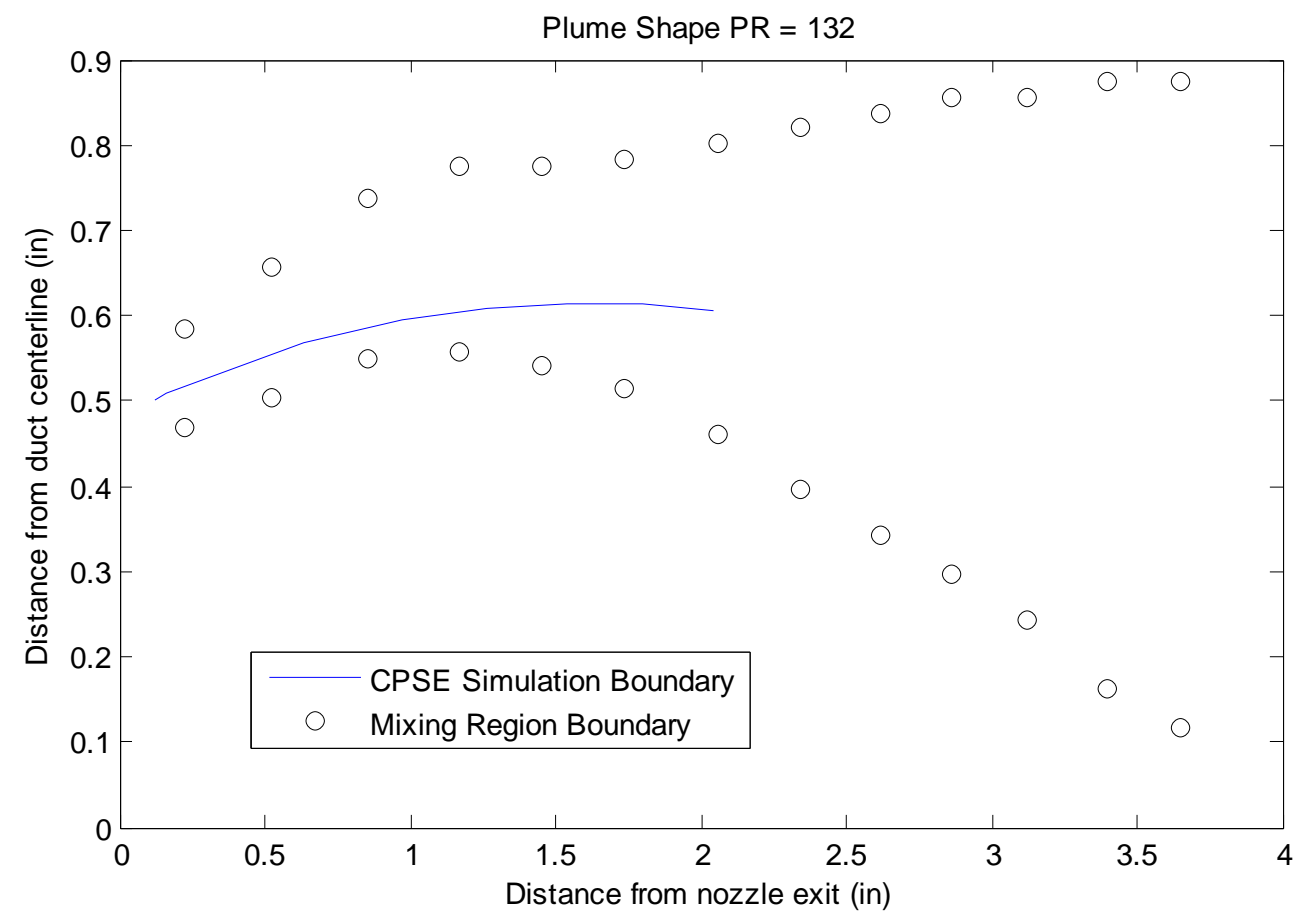

Figure 7-11: Comparison of CPSE simulation to results from Test 60 at $P R=132$ 
The simulation prediction falls within the observed boundary of the mixing region; which would indicate that the mixing layer forms around the inviscid boundary predicted by the simulation. The simulation also shows that the primary plume reaches its maximum expansion point 1.54 inches downstream of the nozzle.

In order to check and see if the simulation is giving an accurate description of the flow field other comparisons must be looked at. The simulation predicts that at a pressure ratio of 132 the secondary Mach number will be 0.989 . During Test 60 , at a pressure ratio of 132, the secondary Mach number was found to be 0.76 . The difference between the predicted secondary Mach number and the actual secondary Mach number is $30 \%$. Figure 7-12 helps to show why the secondary Mach number predicted by the simulation is so high. According to the simulation the primary plume never reaches the secondary flow; meaning that the primary flow has yet to reach the saturated case. The simulation is designed to assume that the flow is choked at the minimum area which in this case is the secondary duct. 


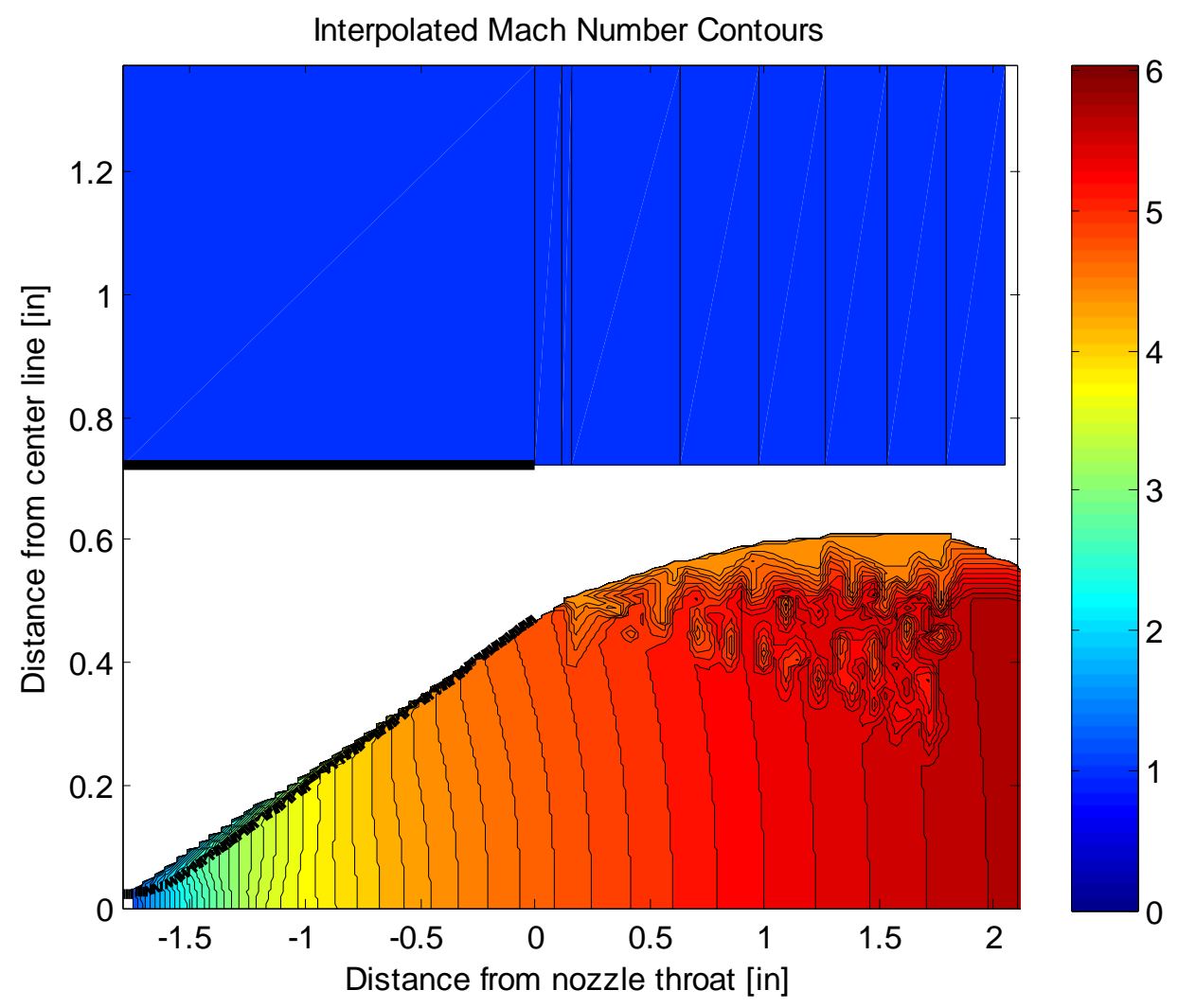

Figure 7-12: Simulation prediction of primary plume for Test 60 with a $P R=132$

The simulation prediction is at odds with the data collected during the test. Figure 7-12 shows what appears to be a shock emanating from the lip of the nozzle. Images taken during the tests show a shock occurring downstream of the nozzle and not at the nozzle lip. This shows that the simulation is not accurately characterizing the flow inside the primary plume. The simulation also predicts that the flow field in the mixing duct will not reach the Fabri choked condition. During Test 60 the secondary Mach number reaches Mach 1 before sharply reducing as the pressure ratio increases. The reduction in secondary Mach number implies that the primary plume is passing through the saturated case and into the Fabri choked case. So the question then becomes, what explains the difference between the simulation and the test? The answer can be found when looking 
at the exit Mach number of the nozzle as predicted by the simulation and measured during testing. The simulation gives an exit Mach number of 4.8, which makes sense because the area ratio of the nozzle is 22 . By looking at isentropic flow tables, it can be found that for gas with a gamma of 1.4, a nozzle area ratio of 22 will create a Mach 4.8 flow $^{19}$. The calculated exit Mach number is 4.28 for this test and pressure ratio. The difference in Mach number is most likely causing the discrepancy in plume shape between the simulation and the test. It makes sense that the test would experience a lower Mach number because the nozzle is non-isentropic. The straight nozzle walls, as well as imperfections in the surface would cause shocks in the nozzle and lower the exit Mach number. In order to try and get a more accurate comparison between the simulation and the tests, the nozzle geometry of the simulation was adjusted to make the Mach number at the exit of the nozzle match what is being recorded in the tests. This adjustment impacts the secondary Mach number of the simulation; however, it does not impact the shock prediction shown in Figure 7-12. This simulation still predicts a shock emanating from the nozzle lip; a shock that is not observed during testing. 


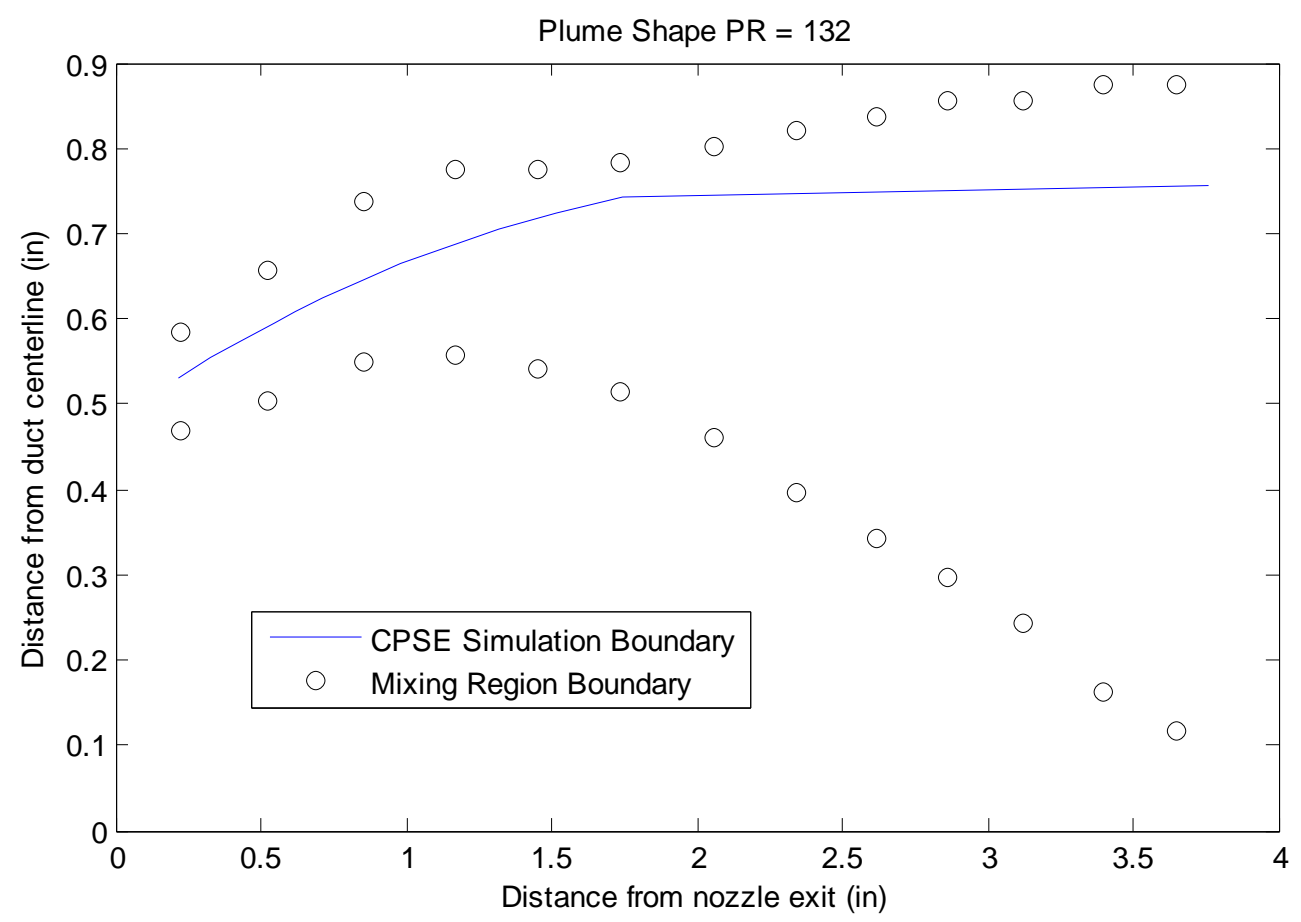

Figure 7-13: Comparison of Simulation and observed boundary layer for Test 60 PR $=132$

Figure 7-13 shows the simulated plume boundary with an adjusted exit Mach number, compared to the observed boundary of the mixing region. In Figure 7-13 the simulation still falls within the observed boundary of the mixing region. However, the simulation is now predicted to expand farther out and into the secondary flow, it also expands farther downstream of the nozzle. The simulation now predicts a secondary Mach number of 0.775 , compared to 0.76 found during the test. The secondary Mach numbers predicted by the simulation and found in the test differ by $1.97 \%$. The secondary Mach number calculation has an error of 3.76\%. The simulation and the test match up within the limits of accuracy of the calculations. Table 7-4 shows the comparison between the secondary Mach number recorded in the tests and calculated by the simulation. Table 7-4 is shown for the other 9 tests in Appendix B. 
Table 7-4: Comparison of Secondary Mach number between Test 60 and Simulation

\begin{tabular}{|r|r|l|r|}
\hline PR & Test & Simulation & \% difference \\
\hline 85 & 0.99 & 0.989 & 0.10 \\
\hline 96 & 0.96 & 0.989 & 2.93 \\
\hline 106 & 0.93 & 0.915 & 1.63 \\
\hline 113 & 0.89 & 0.884 & 0.67 \\
\hline 118 & 0.87 & 0.866 & 0.46 \\
\hline 125 & 0.83 & 0.824 & 0.72 \\
\hline 130 & 0.79 & 0.763 & 3.53 \\
\hline 132 & 0.76 & 0.775 & 1.93 \\
\hline 131 & 0.74 & 0.783 & 5.49 \\
\hline 126 & 0.75 & 0.795 & 5.66 \\
\hline 121 & 0.78 & 0.832 & 6.25 \\
\hline 115 & 0.80 & 0.867 & 7.72 \\
\hline 110 & 0.80 & 0.908 & 11.89 \\
\hline 105 & 0.81 & 0.938 & 66.63 \\
\hline 95 & 0.33 & 0.989 & 70.67 \\
\hline 89 & 0.29 & 0.989 & 69.66 \\
\hline 85 & 0.30 & 0.989 & 82.81 \\
\hline 78 & 0.17 & 0.989 & \\
\hline
\end{tabular}

Table 7-4 shows that at the start of Test 60 , up to the maximum pressure ratio of 132, the observed secondary Mach number and the secondary Mach number predicted by the simulation match up within the $3.76 \%$ limit of accuracy. During the next part of the test the secondary Mach number increases slowly up to 0.81 at a pressure ratio of 105 . The simulation predicts that the secondary Mach number will go back up to Mach 1 . The reason for this difference between the simulation and the test is the plenum chamber. The plenum chamber restricts the amount of secondary mass flow available to the test, which causes the secondary Mach number to stay relatively constant rather than go back to sonic in the duct. Once Fabri choking is no longer occurring in the mixing duct, the secondary Mach number quickly drops and any comparisons with the simulation are invalid. 
Looking back at Figure 7-13, the simulation predicts that the plume will choke 3.76 inches downstream of the nozzle. However, it also shows the plume reaching $98.5 \%$ of its maximum expansion 1.74 inches downstream of the nozzle. The plume is not predicted to have any significant expansion past 1.74 . The oblique shock in the primary flow starts 1.55 inches downstream of the nozzle exit. The oblique shock forms right before the location where the primary plume decreases its rate of expansion. In fact it is the oblique shock which turns the flow; since flow that passes over an oblique shock turns towards the shock. Figure 7-14 shows that the relationship between the simulation results and the oblique shock hold true for multiple pressure ratios. Notice that the simulation line turns at the same location as the oblique shock forms.
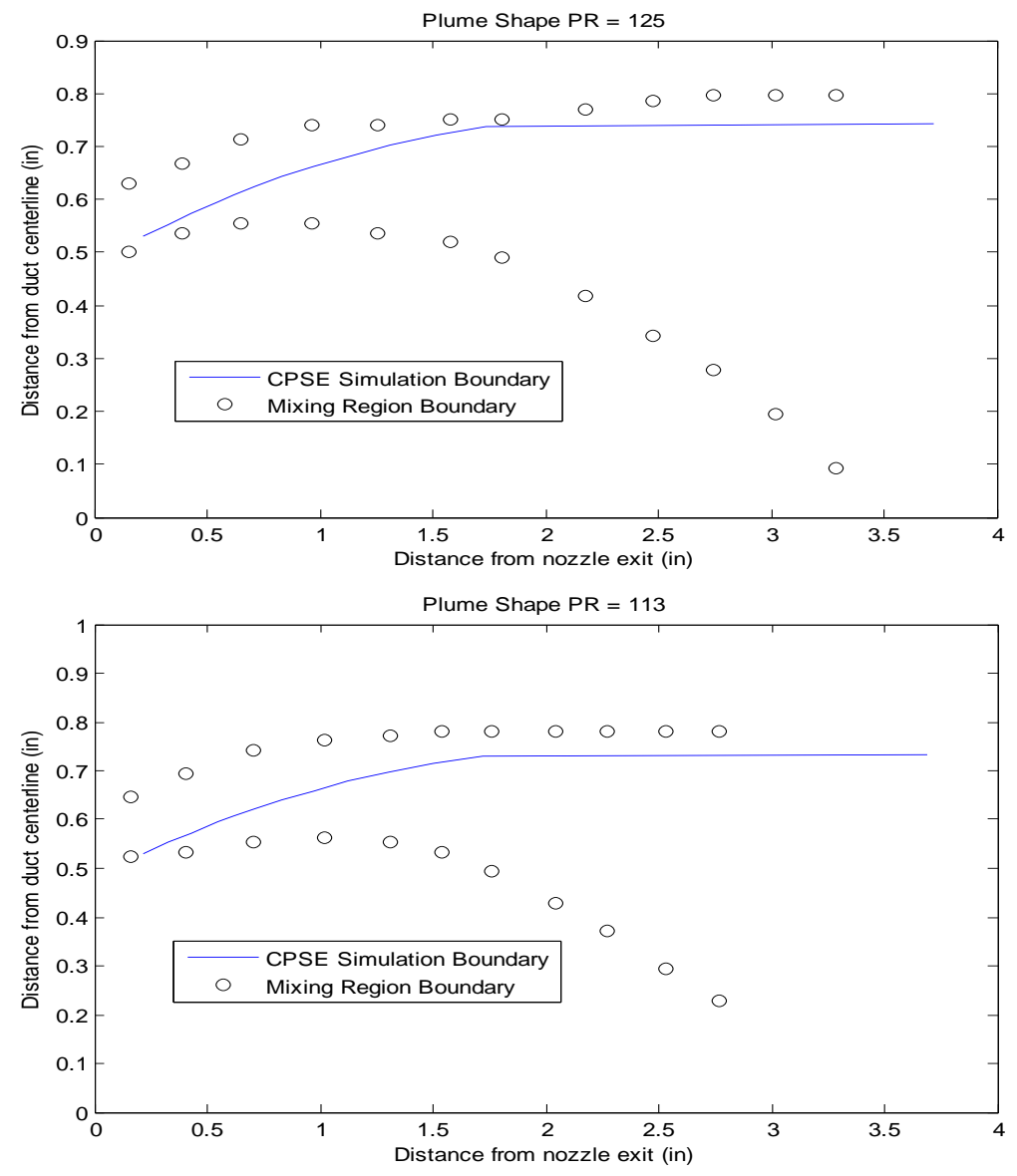

Figure 7-14: Comparison between simulation boundary and observed mixing region 
Figure 7-14 shows the mixing region compared to the plume boundary of the simulation for pressure ratios of 113 and 125 . In both cases the simulation stops making significant expansion between 1.5 and 2 inches downstream of the nozzle exit. The oblique shock also begins 1.5 to 2 inches downstream of the nozzle exit. Theory predicts that the oblique shock will turn the primary flow. The simulation results and oblique shock data are in agreement with this theoretical prediction; as the primary flow predicted by the simulation makes a sharp turn at the start of the oblique shock. This comparison holds true for pressure ratios above 100. When the pressure ratio is below 100 the simulation and the oblique shock no longer match up because Fabri choking is no longer occurring, making the simulation results invalid.

The correlation between simulation results and the oblique shock location give insight into the flow field of the Cal Poly AAR but they do not show where Fabri choking is occurring. In order to help pinpoint the location of Fabri choking, pressure ports were placed along the wall of the mixing duct. These pressure ports record the static pressure at different locations along the wall downstream of the nozzle exit. The critical pressure of the secondary flow can be calculated using isentropic relations and then compared to the pressure measurements taken along the wall of the duct. The location where the wall pressure ports match the critical pressure is the location where Fabri choking is occurring. In the case where the Fabri choke point lies between two pressure ports, linear interpolation was used to determine the approximate location of Fabri choking.

Table 7-5 shows the location of Fabri choking in the mixing duct, referenced from the nozzle exit, calculated using wall pressure points. The table also shows the location where the primary plume turns and the location where the oblique shock forms; both are 
referenced from the nozzle exit. Table 7-5 also shows the distance the simulation predicts that the plume will expand at the Fabri choke point and the distance that the mixing region expands from the centerline of the duct at the Fabri choke point. The Fabri choke data is only recorded for pressure ratios where Fabri choking occurred. The data in Table 7-5 is recorded for each test in Appendix C.

Table 7-5: Comparison of CPSE Simulation to Test 60

\begin{tabular}{|c|c|c|c|c|c|}
\hline \multirow[t]{2}{*}{ PR } & \multirow{2}{*}{$\begin{array}{l}\text { Fabri Choke } \\
\text { location (in) }\end{array}$} & \multirow{2}{*}{$\begin{array}{l}\text { Location of } \\
\text { Oblique Shock } \\
\text { (in) }\end{array}$} & \multirow{2}{*}{$\begin{array}{l}\text { Flow turning } \\
\text { point (in) }\end{array}$} & \multicolumn{2}{|c|}{ Plume Expansion (in) } \\
\hline & & & & Test & Simulation \\
\hline 106 & 1.02 & 1.537 & 1.715 & 0.743 & 0.729 \\
\hline 113 & 1.38 & 1.540 & 1.720 & 0.781 & 0.732 \\
\hline 118 & 1.70 & 1.568 & 1.732 & 0.783 & 0.735 \\
\hline 125 & 1.94 & 1.578 & 1.738 & 0.775 & 0.743 \\
\hline 130 & 2.28 & 1.586 & 1.742 & 0.770 & 0.759 \\
\hline 132 & 2.45 & 1.553 & 1.745 & 0.783 & 0.755 \\
\hline 131 & 2.54 & 1.514 & 1.745 & 0.819 & 0.753 \\
\hline 126 & 2.47 & 1.534 & 1.741 & 0.775 & 0.750 \\
\hline 121 & 2.31 & 1.635 & 1.735 & 0.775 & 0.741 \\
\hline 115 & 2.12 & 1.560 & 1.722 & 0.790 & 0.735 \\
\hline 110 & 1.91 & 1.478 & 1.718 & 0.800 & 0.730 \\
\hline 105 & 1.96 & 1.496 & 1.715 & 0.743 & 0.727 \\
\hline
\end{tabular}

The location of turning point and start of the oblique shock change very little across the different pressure ratios observed in Test 60 . The simulation predicts the turning point lies between 1.7 and 1.75 inches downstream during Test 60. The images of the oblique shock show that it is starting between 1.47 and 1.63 inches downstream of the nozzle exit. Both the turning point in the simulation and the start of the oblique shock change very little over the course of the test.

The Fabri choke point varies from 1.02 inches downstream of the mixing duct at the start of the test to 2.54 inches downstream at its maximum pressure ratio. The test 
results show that the Fabri choke location moves farther downstream as pressure ratios increase, then moves back upstream as the pressure ratio of the test decreases. The Fabri choke point is further from the nozzle exit than the simulation turning point and the start of the oblique shock for the majority of the test. At the beginning of the test the Fabri choke point is located much closer to the nozzle exit than the simulation's predicted turning point. The discrepancy between the test data and simulation is most likely caused by startup transients. The pressure ratio is changing very rapidly at the beginning of the test.

The extent to which the primary plume expands out from the center line of the mixing duct can give insight into the mixing that is occurring between the primary and secondary flows. The simulation expansion values show how much flow area is available to the primary and secondary flows in order to achieve the predicted secondary Mach number. The simulation calculates secondary Mach number based on area ratio. The simulation accurately shows how much the primary plume is expanding in the cases where the secondary Mach number for the simulation and test are in agreement. For each pressure ratio the measured mixing region boundary is farther from the centerline of the mixing duct than the simulation boundary. The actual boundary should extend farther than the simulation prediction because the simulation is an inviscid prediction. The simulation shows how much flow area is needed if the entire secondary flow area is of uniform velocity. In reality the secondary flow is mixing with the primary flow within the mixing region. At this point the two flows are exchanging mass and the uniformity of the flow field is disrupted. This disruption by viscous forces accounts for the difference between the simulation plume boundary and the edge of the mixing region at the Fabri 
choke point. It is important to note that the difference between the mixing region boundary and the simulation plume boundary is relatively small, between 0.01 and 0.07 inches for Test 60 . The small difference is an indicator that there is little mixing occurring between the primary and secondary flows at this point in the mixing duct, because the two flows occupy areas that are close to the areas predicted by inviscid analysis.

The results shown in this section help in describing what is going on in the mixing duct of the Cal Poly AAR. At a location of approximately 1.5 inches downstream of the nozzle an oblique shock forms in the primary plume. This shock causes the primary plume to turn, and reduces the expansion rate of the primary plume. The primary plume continues to expand beyond the start of the oblique shock until reaching the Fabri choke location. The Fabri choke location was found to be as far as 2.5 inches downstream of the nozzle at a pressure ratio of 132 .

\subsection{Transient Considerations}

One way to evaluate the transient conditions in the tests is to look at the secondary Mach number and see how it changes over the course of each test. The secondary Mach number is greatly impacted by the shape of the primary plume. As the plume expands out it lowers the Mach number in the secondary duct. Looking at how quickly the secondary Mach number changes gives an idea as to how fast the primary plume is changing. The main driver of this plume change is the pressure ratio. 
Figure 7-15 shows the secondary Mach number for each of the ten tests as well as the pressure ratio over the course of the tests. The test runs that had the lowest maximum pressure ratios are displayed at the top of the figure, while the highest pressure ratio tests are displayed at the bottom of the figure. By comparing the tests based on maximum pressure ratio it is clear that tests with similar maximum pressure ratios have similar secondary Mach number profiles. Tests 55 and 53 had the lowest maximum pressure ratios at 105 and 106 respectively. In these two tests the secondary Mach number stays close to Mach 1 when the pressure ratio is highest. In Test 60, where the pressure ratio reaches 132 , the secondary Mach number drops to 0.75 . The other tests fall somewhere between these two extremes. One thing that Figure 7-15 consistently shows is that the secondary Mach number decreases as maximum pressure ratio increases. This is due to Fabri choking. When the pressure ratio increases, it causes the primary plume to expand. The outward expansion of the primary plume creates a smaller available flow area in the mixing duct for the secondary flow to pass through. The minimum area is now in the mixing duct and not the secondary duct, as it would be in the saturated case, a situation that causes the Mach number in the secondary duct to decrease. Another parameter that changes with pressure ratio is the length of the test. As the maximum pressure ratio increases the amount of time that Fabri choking is occurring also increases. This is shown in Figure 7-15 by noting the increased length of the flat section of the secondary Mach number profile. 

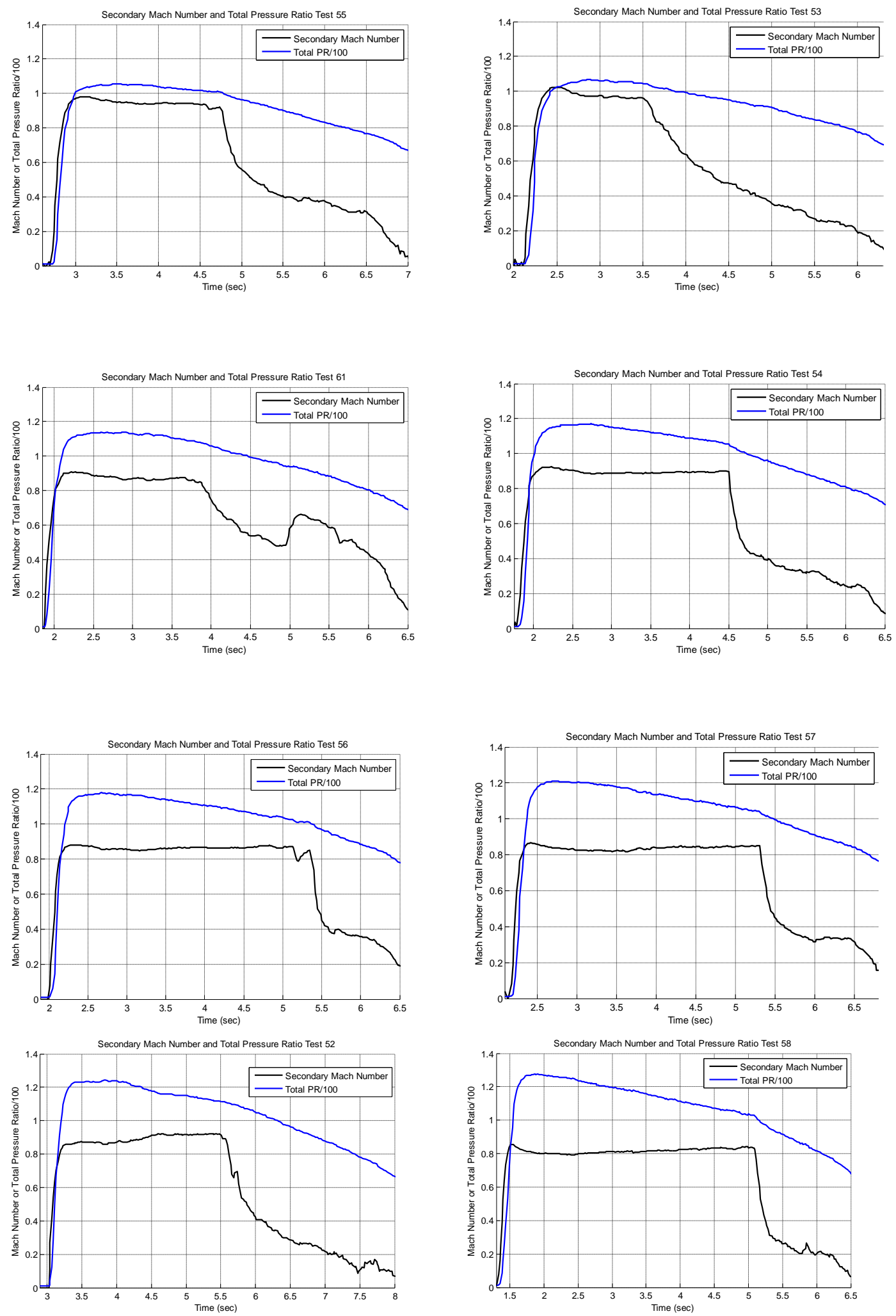

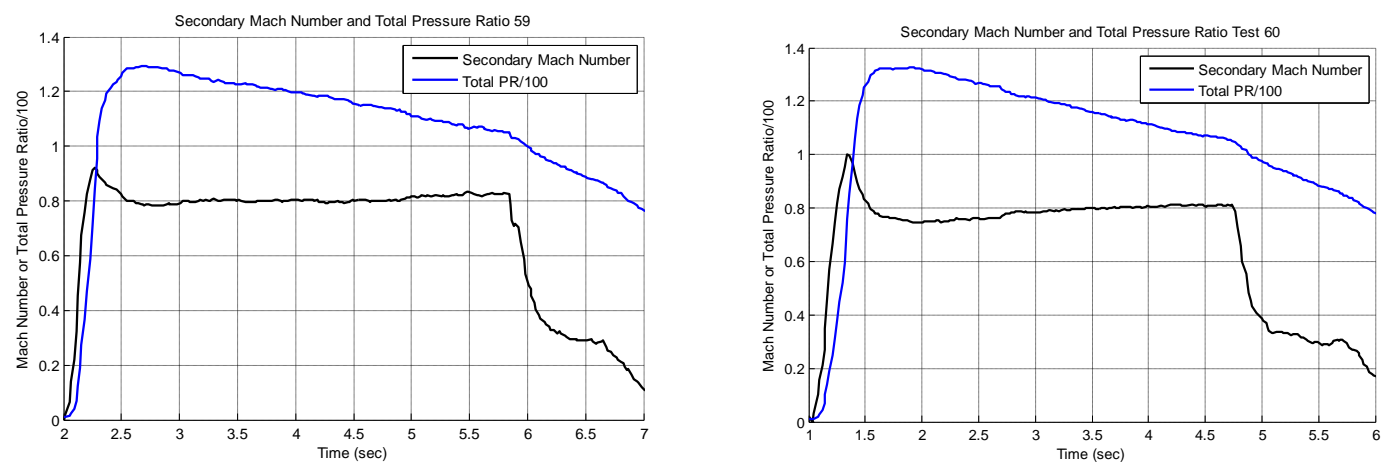

\section{Figure 7-15: Secondary Mach number plots}

One thing to notice when analyzing Figure 7-15 is the shape of the curve that the secondary Mach number creates. For almost every test there is a rise in Mach number at the start of the test, resulting from the startup transients of the test. The sharp rise in Mach number stops at a pressure ratio of around 100 for each test, indicating that the saturated case is occurring at a pressure ratio close to 100 . As the pressure ratio moves above 100 the secondary Mach number begins to drop. The severity of the Mach number drop depends on the maximum pressure ratio of the test. After the drop, the secondary Mach number remains relatively constant for each test, due to the fact that the pressure ratio is not changing very drastically during this portion of the test. There is a 2 to 3 second time span during each test where the secondary Mach number is relatively constant. The nearly constant secondary Mach number does not mean that the tests have reached a steady condition, only that the secondary Mach number is steady. The stagnation pressures of the primary and secondary flows are still changing; however, during that time span they are changing at a similar rate. As the pressure ratio begins to drop back down to 100 the secondary Mach number should pass back through the saturated condition and reach Mach 1. The secondary Mach number does not increase 
back to Mach 1 in any of the tests. The reason for this is the plenum chamber. At the end of the test the plenum has been pumped down and the amount of mass flow available to the secondary flow is being limited, preventing the flow from accelerating back up to Mach 1.

At a certain point each plot shows a sharp drop in the secondary Mach number. At this point the primary flow is unable to pump the plenum down any farther. As a result the mass flow rate of the secondary flow is greatly reduced and the Mach number in the secondary duct drops off with it. In one case, Test 61, the secondary Mach number begins to rise up again. It is believed that this rise was caused by a leak in the plenum allowing more mass flow to enter the secondary duct. The other test that shows a strange anomaly is Test 52. In Test 52 there is no drop in Mach number after the initial rise. The Mach number stays relatively constant at the start of the test then rises up half way through and staying constant again. It is unclear as to why this test did not follow the same trend as the other tests.

\subsection{Plume Characteristics}

A look at the shadowgraph images, taken during the tests, reveals the nature of the primary plume throughout its different operating conditions. At the beginning of each test the primary plume quickly expands outward, since the primary flow is underexpanded. Figure 7-16 gives an illustration of the under-expanded case. 


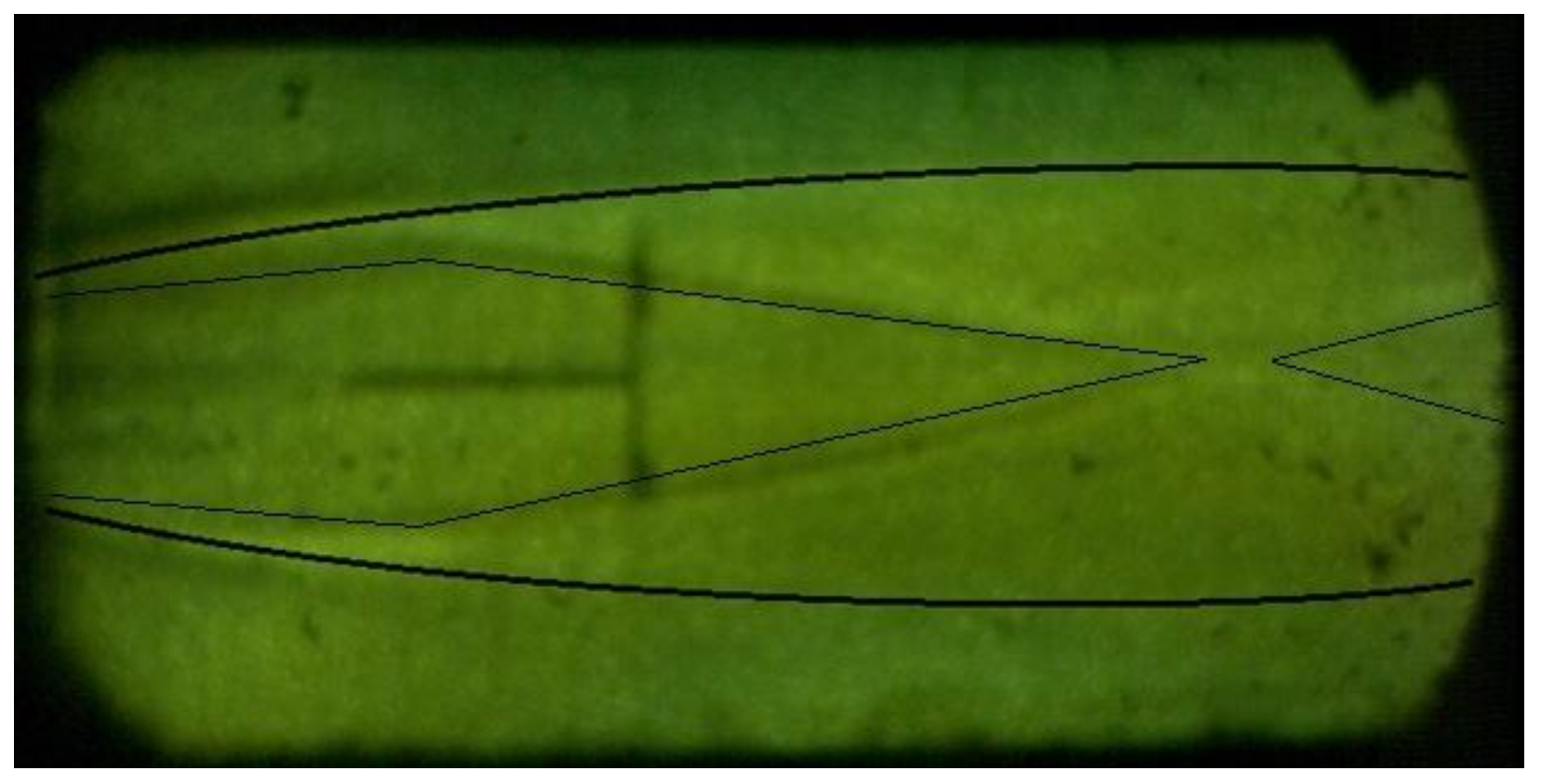

Figure 7-16: Test 60 time 0.6 seconds

The under-expanded primary plume is seen at the beginning of each test. The amount the primary plume expands outward is dependent on the maximum chamber pressure during the test. If the chamber achieves a higher pressure then the plume will expand further outward. It now may be helpful to refer back to Figure 1-9. Figure 1-9 shows the expansion cycles of an under-expanded supersonic primary plume. Figure 716 shows an entire expansion cycle; as the pressure in the chamber drops, the length of each expansion cycle shrinks. Figure 7-17 shows a complete expansion cycle with a complete second cycle. 


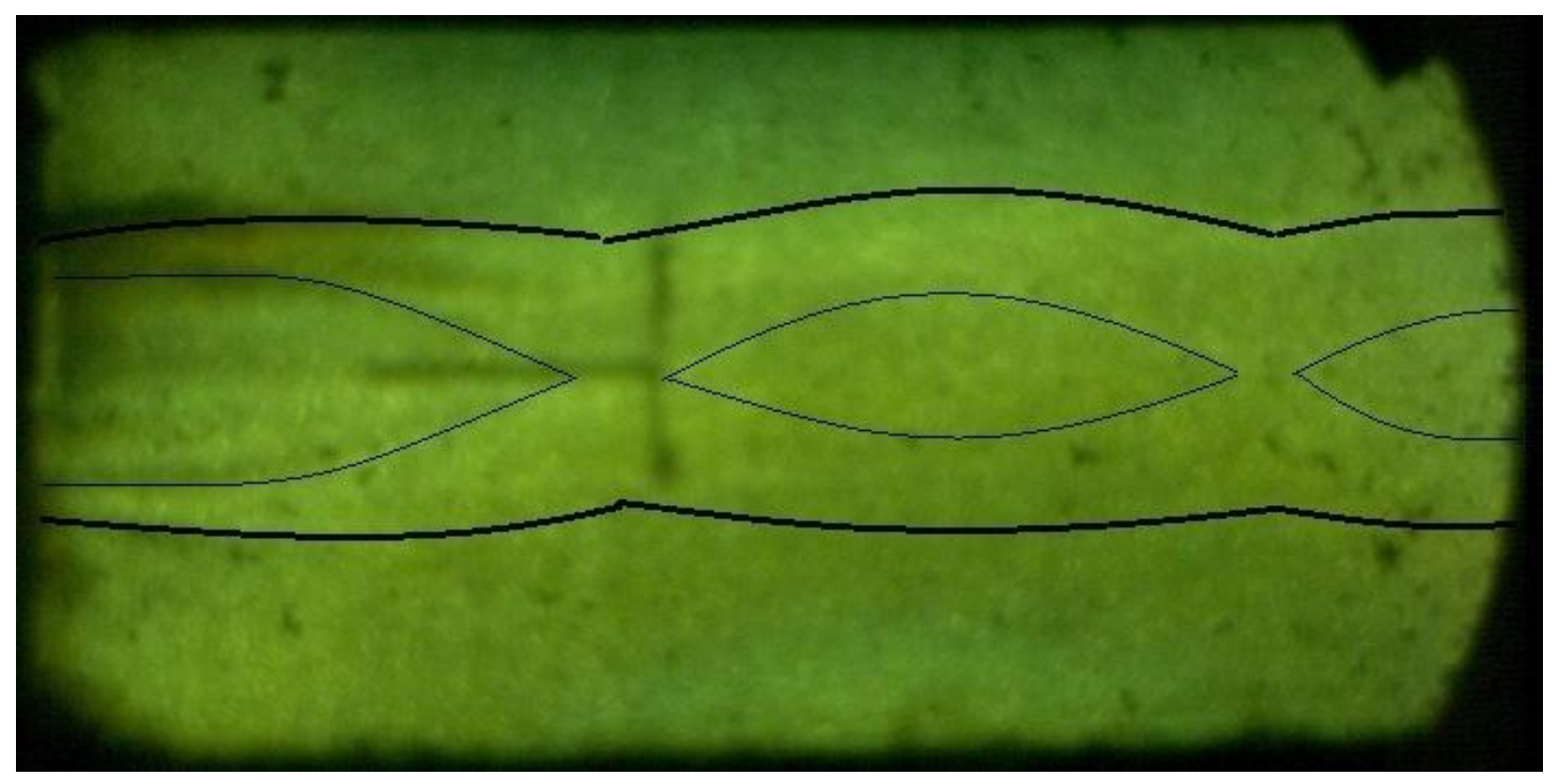

Figure 7-17: Test 60 time 4.85 seconds

At around 4.50 seconds into the test the primary plume shifts from being underexpanded to optimally-expanded. The shift can be seen in Figure 7-17. Notice that the primary plume does not extend out toward the walls, it continues on straight before curving in towards the center line. Once the plume reaches the centerline it expands back out again. The cycle continues downstream of the nozzles with the cycles getting progressively smaller. Figure 7-17 can be compared with Figure 1-9 in order to get a better idea of what is happening.

As the test progresses further, the pressure in the chamber drops significantly, causing the primary plume to be over-expanded. Instead of expanding out toward the wall the plume can be seen collapsing shortly after leaving the nozzle. Figure 7-18 gives an illustration of the plume collapse at the end of the test. 


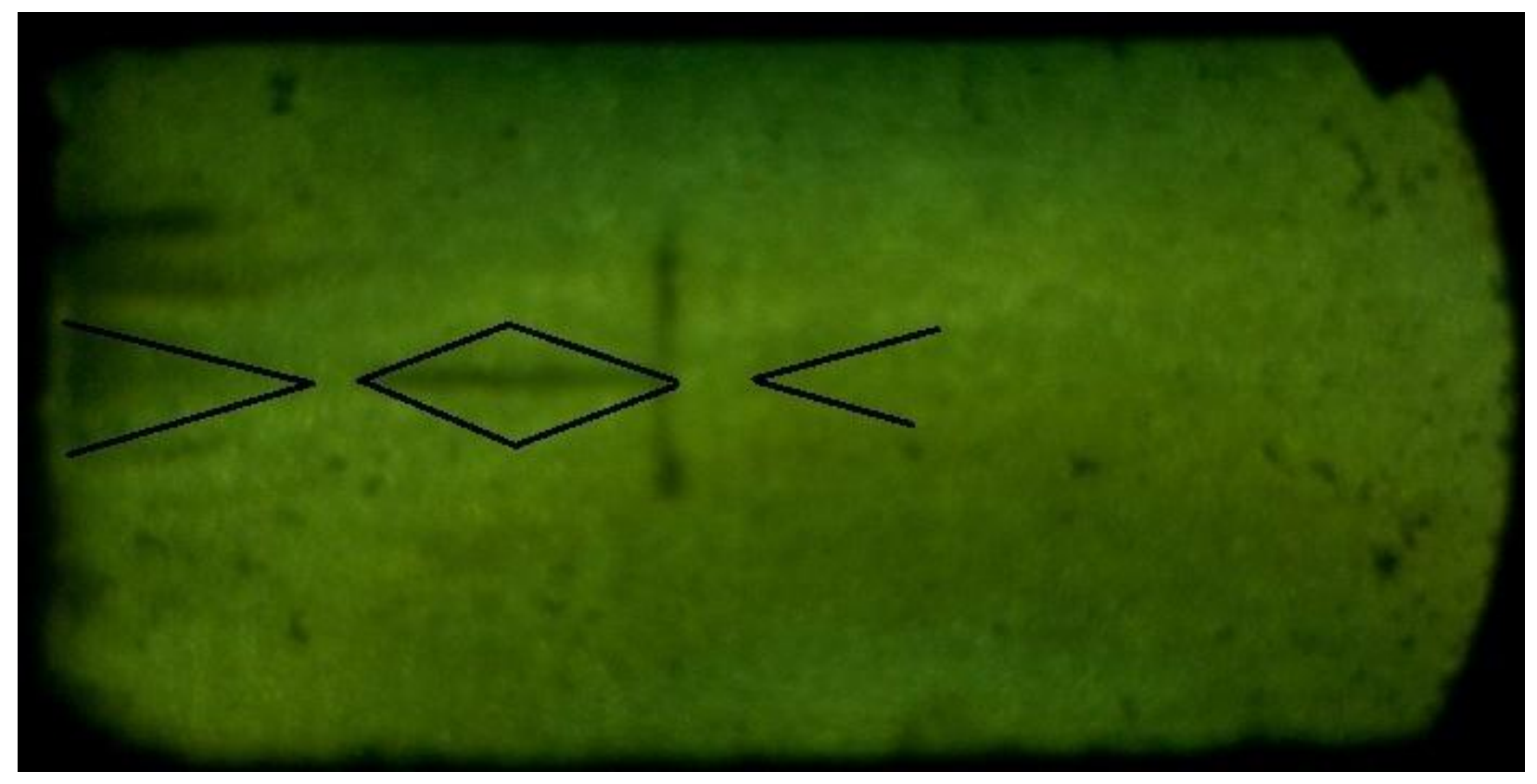

\section{Figure 7-18: Test 60 time 5.80 seconds}

Notice the formation of shock diamonds exiting out the back of the nozzle. Shock diamonds are the classic sign of an over-expanded supersonic jet. Also notice how the length of the cycle has been further reduced. In Figure 7-18 the cycle is a little under an inch in length, whereas at the start of the test the cycle is over 4 inches in length. Also notice that the shock diamonds stop being visible about half way down the picture. The structure of the flow weakens as it moves away from the nozzle making it less clear in the image.

Next Figures 7-16 and 7-18 will be compared to pictures taken during tests conducted by Gist using this same basic hardware. Figure 7-19 shows a picture taken at the beginning of Gist's test, notice how it portrays one large expansion cycle, much like Figure 7-16. 


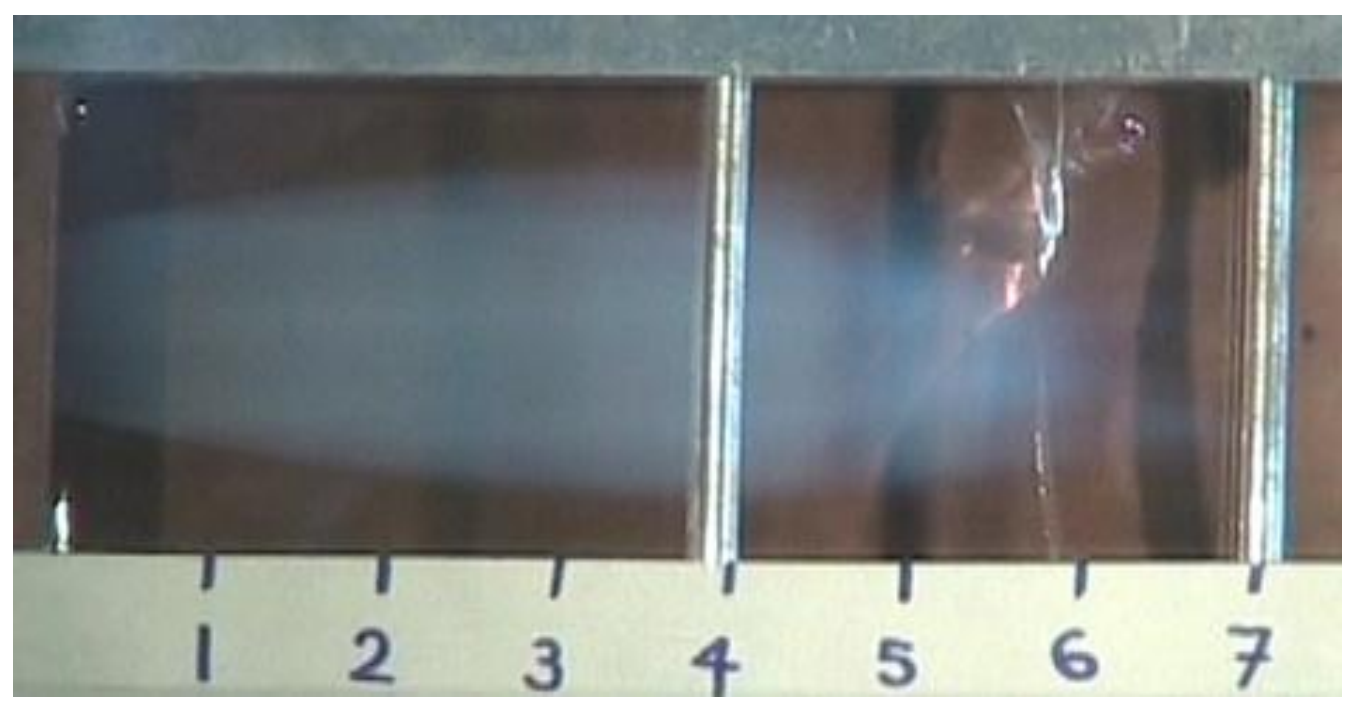

Figure 7-19: Picture of Gist Test II at 2 seconds ${ }^{5}$

Towards the end of Gist's tests he observes a collapse of the primary plume.

Figure 7-20 shows the image that Gist captured of this collapse. Notice the similarities to Figure 7-18, both figures show a shock diamond pattern exiting the nozzle.

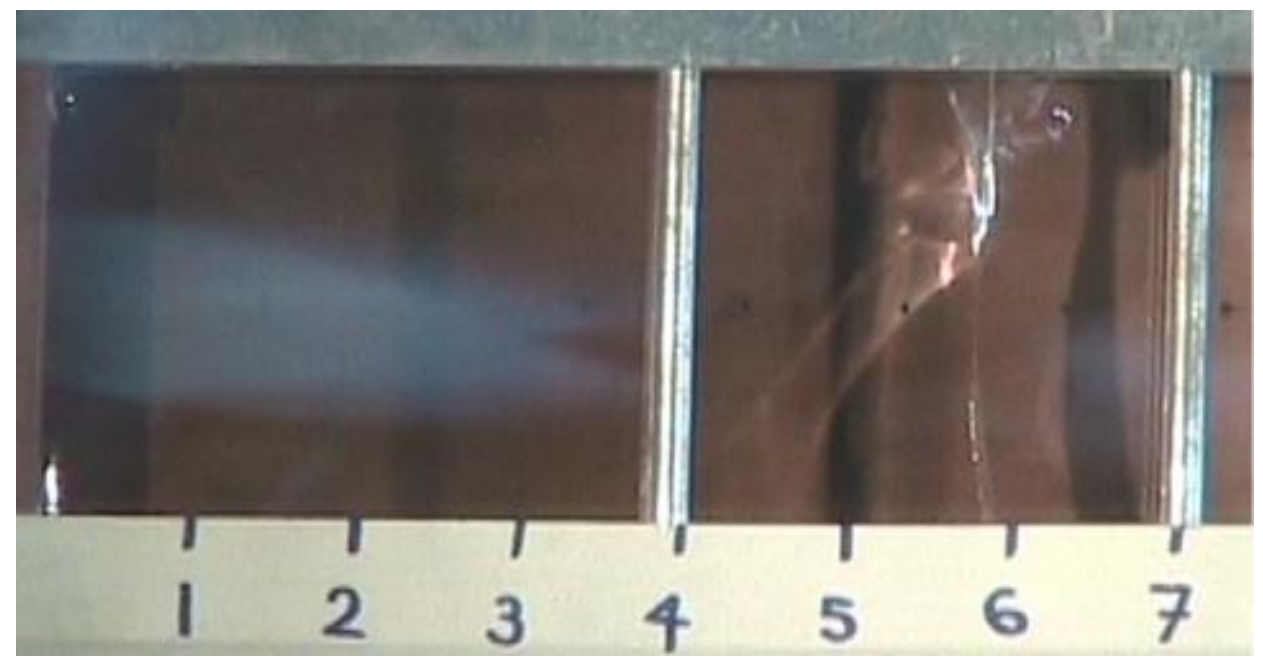

Figure 7-20: Picture of Gist Test II at 11 seconds ${ }^{5}$

The similarities in these figures show that there is a measure of consistency between the old and new tests conducted using the Cal Poly AAR. 
Each of the test runs analyzed in this paper follow the same pattern as Test 60. The plume starts out under-expanded before moving to an optimally expanded then overexpanded condition. The Figures7-21 through 7-24, show shadowgraph images from Tests 52-61 at various times during the test.

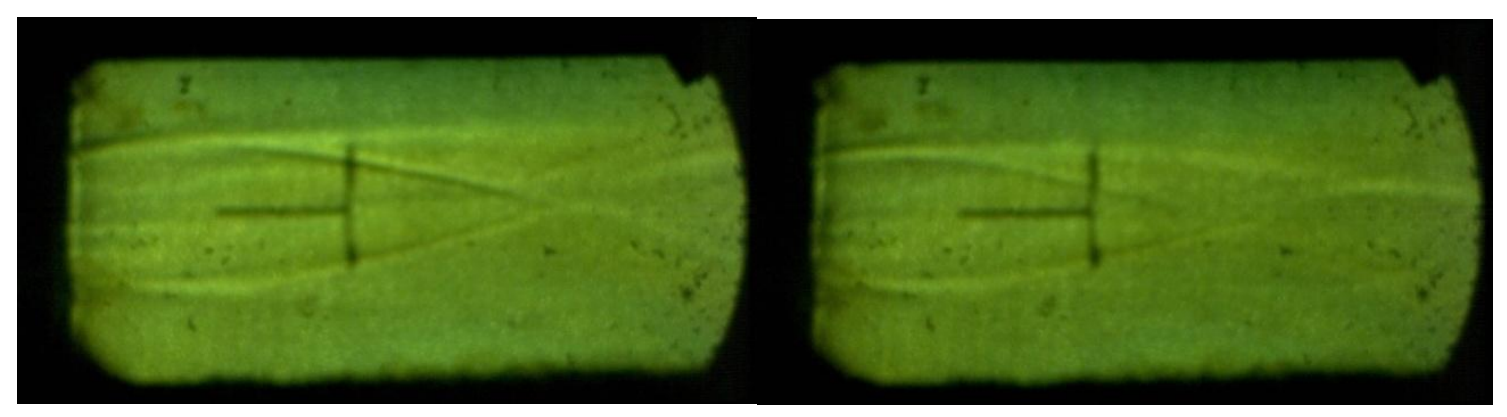

Figure 7-21: Test 53 (Left) time 0.29 seconds; Test 55 (Right) time 2.73 seconds

Tests 53 and 55 were low pressure tests with a maximum pressure ratio of 106 and 105. The low maximum pressure caused the tests to be much shorter and the plume to not expand as far to the wall. The picture on the left shows the flow to be underexpanded. About 2 and a half seconds later the figure on the right shows the flow near the optimally-expanded condition. 


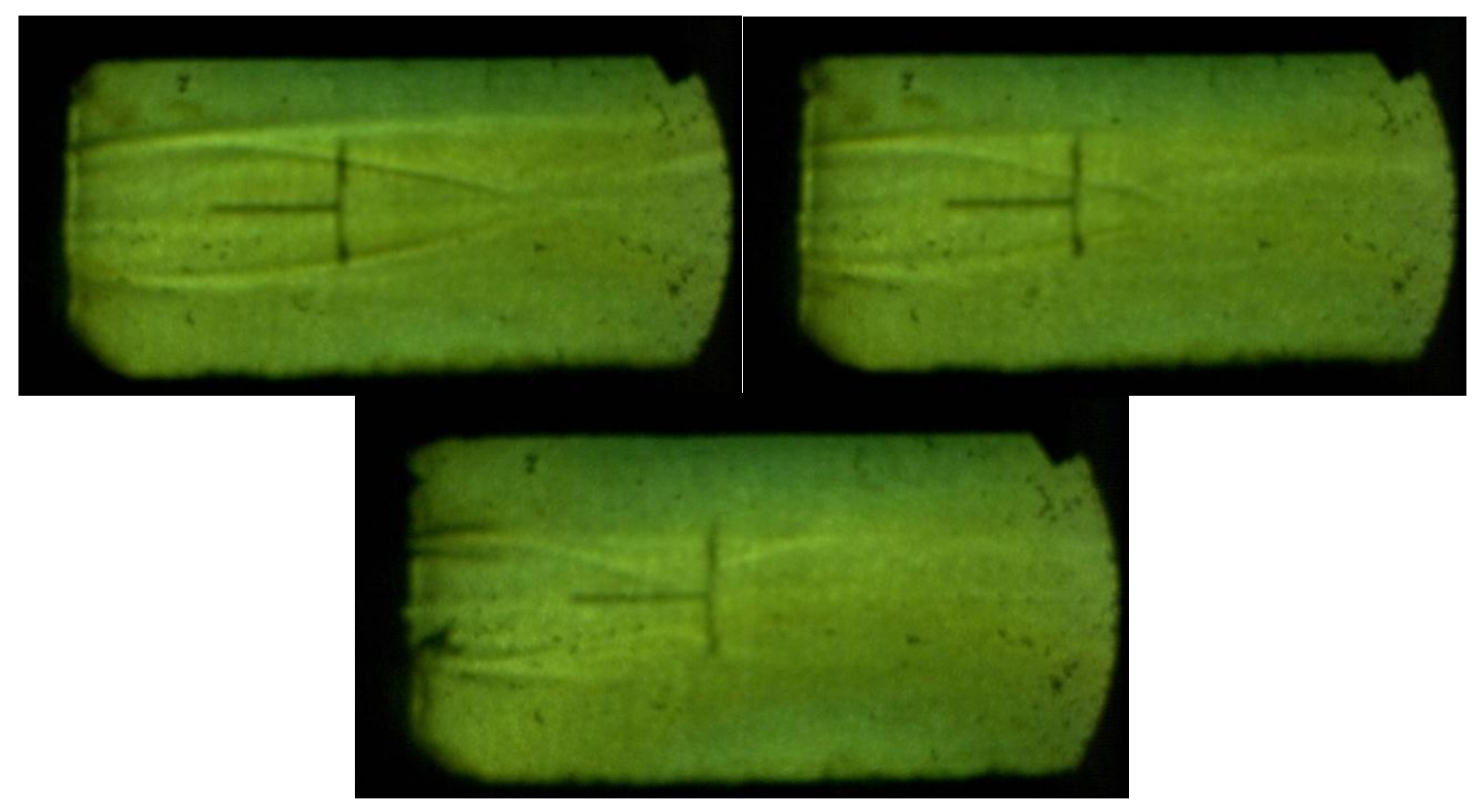

Figure 7-22: Test 54 (Left) time 0.79 seconds; (Right) Test 56 time 3.45 seconds; (bottom) Test 61 time 4.03 seconds

Tests 54, 56 and 61 reached maximum pressure ratios of 116, 116 and 113 respectively. The higher chamber pressure caused a larger pressure ratio and a longer test. The picture on the top left shows a primary plume that is under-expanded. Notice how the mixing layer moves out toward the edge of the mixing duct. The picture on the top right shows a primary plume that is slightly under-expanded; the plume is still moving out but not very far. The bottom picture shows the optimally-expanded case where the flow comes straight out from the nozzle and does not expand toward the wall.

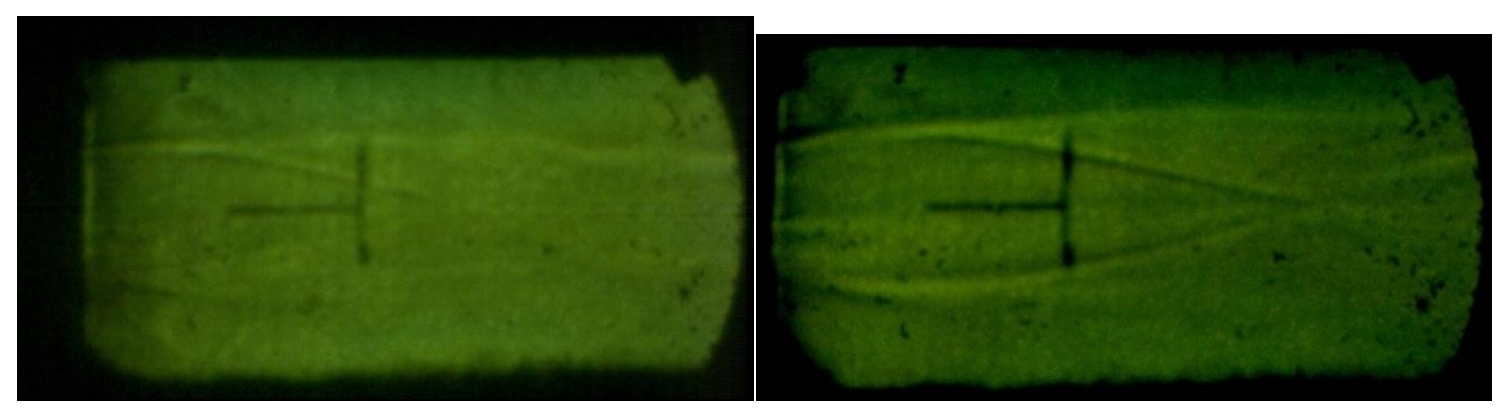

Figure 7-23: Test 57 (Left) time0.13; (Right) Test 52 seconds time 0.75 seconds 
Tests 52 and 57 reached a maximum pressure ratio of 123 and 120. The picture on the left shows a primary plume that is optimally-expanded as the test begins. It also shows a shock in the secondary duct. The shock is present at the start of each test and shows that the secondary flow is reaching sonic conditions in the mixing duct. The picture on the right shows a primary plume that is under-expanded causing the plume to expand outward toward the wall of the mixing duct.

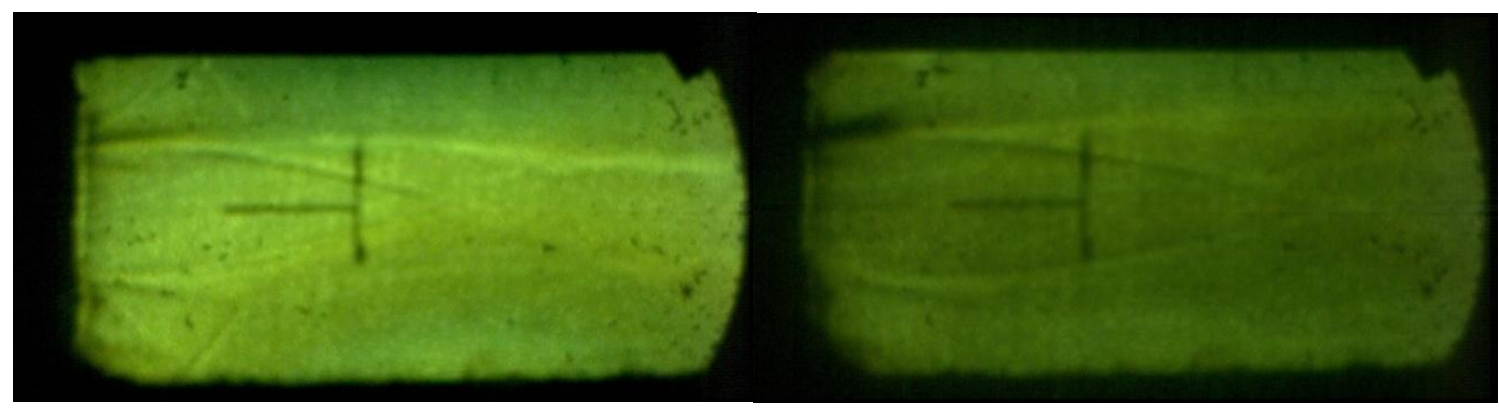

Figure 7-24: Test 59 (Left) time 0.17 seconds; (Right) Test 58 time 1.10

Tests 58 and 59 reached maximum pressure ratios of 127 and 128. Once again the picture on the left shows the presence of shocks in the secondary flow within the mixing duct. The picture on the right shows a primary plume that is under-expanded. 


\section{Conclusion}

During the course of this research shadowgraph images were used to evaluate the interactions between the primary and secondary flows of the Cal Poly AAR. The region from the exit of the nozzle to 5 inches downstream was viewed in the images. The primary and secondary flows were both unsteady, and the maximum pressure ratio reached in a test was 132 . The images show the formation of an oblique shock in the primary flow. A prediction of the shock wave angle was developed using isentropic theory. The predictions of the shock wave angle match the observed measurements to within $8.41 \%$. The shock occurs in the same location, approximately 1.5 inches downstream of the nozzle exit, for all pressure ratios where Fabri choking is occurring. The images also show the oblique shock reflecting off the symmetry plane at the centerline of the mixing duct. The observations of the shock reflection agree with other experiments conducted on the reflection of weak shocks.

The images also show the size of the mixing region that develops between the primary and secondary flows. The images show that the region of viscous influence is large; taking up as much as $25 \%$ of the cross-sectional area of the duct at 2 inches downstream of the nozzle; showing that viscous forces should play a significant role in the development of theoretical tools aimed at predicting conditions within the mixing duct of the Cal Poly AAR. The size of the mixing region appears to be in line with assumptions made by Gist who accounts for the mixing region by setting it equal to the nozzle lip thickness of 0.25 inches. 
The static pressure was recorded along the wall of the mixing duct from 2 inches to 6.5 inches downstream of the nozzle exit. The static pressure reaches a minimum value in the mixing duct of the AAR. The minimum wall pressure corresponds to the location where the oblique shock reflects off the centerline of the mixing duct. Beyond the reflection point the static pressure begins to increase along the wall. The static pressure along the wall was also used to locate where Fabri choking is occurring within the mixing duct. Analysis shows that choking is occurring between 1 inch and 2.5 inches downstream of the nozzle exit.

An analysis of the Mach number in the secondary duct shows the transient nature of the test conditions. At the beginning of the tests the secondary Mach number changes rapidly. Once the pressure ratio reaches its maximum value for the test the secondary Mach number levels out. The maximum pressure ratio corresponds to the lowest secondary Mach number. Once the pressure ratio drops below 100 the Mach number drops off rapidly, suggesting that the saturated condition is occurring around a pressure ratio of 100 .

The shadowgraph images were also used to look at the shape of the primary plume within the mixing duct. The data from the images was compared to the 2-D CPSE simulation. The results of the comparison showed that the simulation gives an accurate description of the shape of the primary plume. The simulation prediction falls within the observed mixing region for the majority of cases. However, the simulation does not accurately predict the shocks observed in the primary flow during testing. The simulation predicts that an oblique shock will emanate from the lip of the nozzle. Observations show that the oblique shock starts downstream of the nozzle. Also, the simulation does 
not do a good job of capturing the velocity of the secondary flow. The discrepancy is due to the simulation's inability to accurately model the performance of the nozzle. It is believed that the inaccuracies in the simulation come from the lack of a viscous component within the model, and the presence of the plenum chamber during testing. For these reasons the CPSE simulation is not suited for predicting the conditions in the Cal Poly AAR.

The simulation can be modified in order to help evaluate test results obtained using the Cal Poly AAR. The modification involves matching the exit Mach number of the nozzle to conditions measured during testing. The modified simulation is able to predict the secondary Mach number of the tests, within 3.7\% of the actual value, at the beginning of the tests. At the end of the tests the presence of the plenum chamber prevents an accurate prediction by the simulation. The modified simulation also predicts that the primary plume will finish the majority of its expansion at around 1.7 inches downstream of the nozzle exit. The simulation shows that the inviscid boundary between the primary and secondary flow is within 0.1 inch of the boundary of the mixing region seen in the shadowgraph images. The results obtained from the modified CPSE simulation show that it does have value as a tool for evaluating and understanding results that have already been obtained through testing. 


\section{$9 \quad$ Future Work}

One question raised during the course of this investigation has to do with the effect of a thick nozzle lip on shear layer growth. Tests should be performed with nozzles of various lip thickness in order to determine the effect that varying the thickness has on the mixing region and entrainment ratio. Rockets cannot operate with thin lips, so understanding the effect of the thick lip is critical to understand AAR performance.

All of the tests conducted for this investigation occurred under transient conditions. The stagnation pressure of the primary and the secondary flows were changing throughout the course of the test. The accuracy of these tests could be improved if the primary stagnation pressure of the rocket could be held constant throughout the test. Creating a steady primary flow would allow a more accurate investigation into the size and shape of the mixing region, and the relationship between pressure ratio and entrainment ratio. Tests conducted under steady conditions would also be easier to correlate with the CPSE simulation, or any other predictive model. Working to create a steady state test should be the next step in the research conducted on the Cal Poly AAR.

The images analyzed in this paper are only capable of showing the relative size of the mixing region. They give insight into how large the region of viscous influence is, but are unable to provide exact quantitative measures of the shear layer. Shadowgraph images do not provide information on the flow characteristics within the shear layer. In order to accurately understand how much mixing is occurring between the primary and secondary flows, turbulence data must be taken from within the mixing layer. 
Turbulence data can be obtained through the use of hot wire anemometry or Laser Doppler Anemometry. Obtaining detailed turbulence data is the next level of analysis that needs to be conducted on the mixing layer in order to get a complete picture of the mixing duct flow field.

Future work in simulating conditions in the mixing region should include a full viscous solution. The mixing region has been shown to be large in the Cal Poly AAR and it should not be neglected in future simulations. The pressure data and images gathered during these tests provide plenty of data for validating future computational results. 


\section{References}

1. "Hypersonics: Research and Development", National Aeronautics and Space Administration Marshall Space Flight Center Huntsville, FS-2003-01-01-MSFC, Huntsville, Alabama, Jan. 2003.

2. Peebles, Curtis, Road to Mach 10, American Institute of Aeronautics and Astronautics, Inc., Reston, VA, 2008.

3. “X-51 Waverider makes historic hypersonic flight”, U.S. Air Force, http://www.af.mil/news/story.asp?id=123206525 [Cited 26 May 2010].

4. Law, Peter, "SR-71 Propulsion system” Spring 1991, http://www.enginehistory.org/Convention/2005/Presentations/LawPete/SR71Propulsion1.pdf [Cited 23 Feb. 2011].

5. Gist, Ryan, "Examination of Flow Field Characteristics and Fabri-Choking of a 2D Air Augmented Rocket”, California Polytechnic State University, Jan. 2007.

6. Thomas, Scott, Donald, Palac, Charles, Trefny, and Joseph, Roche, "Performance Evaluation of the NASA GTX RBCC Flowpath", Fifteenth International Symposium on Airbreathing Engines, NASA/TM-2001-210953, NASA Glenn Research Center, Cleveland, OH.

7. Hueter, Uwe, "Creating an airline to the stars ", Aerospace America, April, 1999.

8. Addy, L.A., "On the Steady State and Transient Operating Characteristics of Long Cylindrical Shroud Supersonic Ejectors”, Ph. D. Dissertation, University of Illinois, University Microfilms, Inc., Ann Arbor, MI., 1963. 
9. Fabri, J., and Paulon, J., "Theory and Experiments on Supersonic Air-to-Air Ejectors", NACA TM 1410, Sept 1956.

10. Dimotakis, P.E., "Turbulent Free Shear Layer Mixing and Combustion", HighSpeed Flight Propulsion Systems, Progress in Astronautics and Aeronautics, edited by Murthy, S.N.B., Curran, E.T., Vol. 137, pp. 265-340.

11. Birkhoff, Zarantonello, Jets, wakes, and cavities, Academic Press inc., New York, NY, 1957, pp. 309-310.

12. Saad, Michel, Two-Dimensional Waves, Prentice Hall, Englewood Cliffs, NJ, 1985.

13. Schetz, Joseph, Foundations of Boundary Layer Theory for Momentum, Heat, and Mass Transfer, Prentice Hall Inc., Englewood Cliffs, NJ , 1984, pp. 220-221.

14. Settles, G.S., Schlieren and Shadowgraph Techniques, Springer New York, 2001.

15. Fabri, J., and Siestrunk, "Supersonic Air Ejectors", Advances in Applied Mechanics, edited by Von Karman and Dryden, Academic Press, Vol. 5, 1958, pp.1-35.

16. Papamoschou, D., "Analysis of Partially Mixed Supersonic Ejectors”, Journal of Propulsion and Power, Vol. 12, No. 4, July 1996.

17. Foster, Trevor James, "Rectangular Ducted Methane/GOX Thruster", California Polytechnic State University, Feb. 2008.

18. Morham, Brett G., "Numerical Examination of Flow Field Characteristics and Fabri Choking of 2D Supersonic Ejectors", California Polytechnic State University, Jan. 2010. 
19. Zucrow, Maurice, and Hoffman, Joe, Gas Dynamics Volume 1, John Wiley \& Sons, Hoboken, NJ, 1976.

20. "Shock Diamonds and Mach Discs", Aerospaceweb, http://www.aerospaceweb.org/ [cited 2006].

21. "PX-302 Pressure Transducer Specifications Sheet”, Omega Engineering, http://www.omega.com/Pressure/pdf/PX302.pdf [cited 12 Oct. 2010].

22. Hornung, H.G., "Oblique shock reflection from an axis of symmetry", J. Fluid Mech, Vol. 409, 2000, pp 1-12. 


\section{Appendix}

\subsection{Appendix A}

Comparison of Oblique shock wave angles for Test 52

\begin{tabular}{|c|c|c|c|}
\hline Pressure Ratio & $\begin{array}{l}\text { Observed Angle } \\
\text { (deg) }\end{array}$ & $\begin{array}{l}\text { Calculated Angle } \\
\text { (deg) }\end{array}$ & $\%$ Difference \\
\hline 81 & 18.14 & 14.91 & 17.79 \\
\hline 91 & 18.40 & 14.70 & 20.13 \\
\hline 99 & 16.02 & 14.36 & 10.34 \\
\hline 105 & 18.24 & 14.10 & 22.66 \\
\hline 113 & 15.12 & 13.68 & 9.52 \\
\hline 119 & 15.17 & 13.49 & 11.07 \\
\hline 122 & 16.41 & 13.36 & 18.53 \\
\hline 123 & 14.92 & 13.41 & 10.10 \\
\hline 118 & 16.00 & 13.41 & 16.16 \\
\hline 112 & 22.44 & 13.81 & 38.45 \\
\hline 106 & 23.93 & 15.63 & 34.67 \\
\hline 99 & 19.66 & 17.04 & 13.32 \\
\hline 94 & 19.86 & 17.44 & 12.19 \\
\hline 88 & 18.69 & 18.11 & 3.13 \\
\hline
\end{tabular}

Comparison of Oblique shock wave angles for Test 53

\begin{tabular}{|r|r|r|r|}
\hline Pressure Ratio & \multicolumn{2}{l|}{$\begin{array}{l}\text { Observed Angle } \\
(\mathrm{deg})\end{array}$} & $\begin{array}{l}\text { Calculated Angle } \\
(\mathrm{deg})\end{array}$ \\
\hline 70 & 14.61 & 15.02 & 2.81 \\
\hline 78 & 11.93 & 15.27 & 27.99 \\
\hline 88 & 13.65 & 14.57 & 6.72 \\
\hline 98 & 13.98 & 13.95 & 0.19 \\
\hline 104 & 16.46 & 14.10 & 4.31 \\
\hline 106 & 13.00 & 13.65 & 9.69 \\
\hline 103 & 12.31 & 13.50 & 15.36 \\
\hline 96 & 18.99 & 16.07 & 12.10 \\
\hline 91 & 19.34 & 17.00 & 26.38 \\
\hline 86 & 24.31 & 17.89 & 22.28 \\
\hline 81 & 16.08 & 19.67 & \\
\hline
\end{tabular}


Comparison of Oblique shock wave angles for Test 54

\begin{tabular}{|r|r|r|r|}
\hline Pressure Ratio & $\begin{array}{l}\text { Observed Angle } \\
(\mathrm{deg})\end{array}$ & $\begin{array}{l}\text { Calculated Angle } \\
(\mathrm{deg})\end{array}$ & \% Difference \\
\hline 67 & 17.00 & 15.55 & 8.54 \\
\hline 79 & 15.39 & 14.80 & 6.71 \\
\hline 88 & 13.54 & 14.45 & 14.46 \\
\hline 95 & 12.35 & 14.14 & 14.87 \\
\hline 104 & 12.20 & 14.02 & 12.72 \\
\hline 111 & 15.57 & 13.59 & 13.05 \\
\hline 116 & 15.54 & 13.51 & 12.44 \\
\hline 113 & 15.77 & 13.80 & 21.18 \\
\hline 107 & 17.55 & 13.83 & 11.70 \\
\hline 100 & 17.70 & 15.62 & 10.84 \\
\hline 94 & 19.07 & 17.00 & 12.16 \\
\hline 89 & 16.27 & 18.25 & 4.93 \\
\hline 83 & 18.21 & 19.11 & 4.78 \\
\hline 78 & 19.14 & 20.06 & \\
\hline
\end{tabular}

Comparison of Oblique shock wave angles for Test 55

\begin{tabular}{|r|r|r|r|}
\hline Pressure Ratio & $\begin{array}{l}\text { Observed Angle } \\
(\mathrm{deg})\end{array}$ & $\begin{array}{l}\text { Calculated Angle } \\
(\mathrm{deg})\end{array}$ & \% Difference \\
\hline 79 & 21.63 & 15.23 & 29.55 \\
\hline 85 & 15.93 & 14.80 & 7.09 \\
\hline 89 & 14.20 & 14.58 & 2.67 \\
\hline 97 & 17.53 & 14.11 & 2.51 \\
\hline 102 & 14.13 & 13.78 & 3.14 \\
\hline 105 & 13.32 & 13.74 & 9.66 \\
\hline 102 & 15.26 & 13.79 & 9.00 \\
\hline 95 & 19.46 & 17.71 & 7.91 \\
\hline 90 & 16.75 & 18.08 & 6.40 \\
\hline 83 & 18.40 & 19.58 & 5.86 \\
\hline 78 & 19.39 & 20.53 & \\
\hline
\end{tabular}


Comparison of Oblique shock wave angles for Test 56

\begin{tabular}{|r|r|r|r|}
\hline Pressure Ratio & \multicolumn{2}{l|l|}{$\begin{array}{l}\text { Observed Angle } \\
\text { (deg) }\end{array}$} & $\begin{array}{l}\text { Calculated Angle } \\
\text { (deg) }\end{array}$ \\
\hline 76 & 18.09 & 14.91 & 17.59 \\
\hline 86 & 17.09 & 14.37 & 15.92 \\
\hline 94 & 13.92 & 14.49 & 4.11 \\
\hline 99 & 15.03 & 14.20 & 5.52 \\
\hline 103 & 14.47 & 14.09 & 2.59 \\
\hline 107 & 13.64 & 13.70 & 0.44 \\
\hline 113 & 14.70 & 13.60 & 7.50 \\
\hline 116 & 17.60 & 13.34 & 24.17 \\
\hline 113 & 12.33 & 13.61 & 10.36 \\
\hline 108 & 14.75 & 13.50 & 8.46 \\
\hline 103 & 12.74 & 13.98 & 9.75 \\
\hline 96 & 17.57 & 16.60 & 5.52 \\
\hline 90 & 20.03 & 17.18 & 14.23 \\
\hline 84 & 20.69 & 19.04 & 7.97 \\
\hline 79 & 31.25 & 19.01 & 39.17 \\
\hline
\end{tabular}

Comparison of Oblique shock wave angles for Test 57

\begin{tabular}{|c|c|c|c|}
\hline Pressure Ratio & $\begin{array}{l}\text { Observed Angle } \\
\text { (deg) }\end{array}$ & $\begin{array}{l}\text { Calculated Angle } \\
\text { (deg) }\end{array}$ & $\%$ Difference \\
\hline 84 & 14.63 & 14.37 & 1.75 \\
\hline 93 & 15.85 & 14.51 & 8.44 \\
\hline 100 & 13.32 & 14.37 & 7.81 \\
\hline 105 & 13.76 & 14.09 & 2.39 \\
\hline 111 & 14.34 & 13.74 & 4.14 \\
\hline 118 & 13.17 & 13.57 & 3.05 \\
\hline 120 & 12.71 & 13.44 & 5.77 \\
\hline 118 & 15.59 & 13.49 & 13.49 \\
\hline 113 & 14.17 & 13.66 & 3.53 \\
\hline 108 & 12.35 & 14.05 & 13.74 \\
\hline 102 & 16.30 & $\begin{array}{l}14.44 \\
\end{array}$ & 11.37 \\
\hline 96 & 13.08 & 16.75 & 28.11 \\
\hline 90 & 17.97 & 17.95 & 0.11 \\
\hline 85 & 16.38 & 19.46 & 18.76 \\
\hline
\end{tabular}


Comparison of Oblique shock wave angles for Test 58

\begin{tabular}{|r|r|r|r|}
\hline Pressure Ratio & \multicolumn{2}{l|}{$\begin{array}{l}\text { Observed Angle } \\
(\mathrm{deg})\end{array}$} & $\begin{array}{l}\text { Calculated Angle } \\
(\mathrm{deg})\end{array}$ \\
\hline 84 & 16.08 & 14.32 & 10.96 \\
\hline 95 & 14.66 & 14.37 & 1.95 \\
\hline 103 & 13.19 & 14.11 & 7.01 \\
\hline 109 & 13.50 & 14.01 & 3.81 \\
\hline 118 & 12.23 & 13.65 & 11.61 \\
\hline 123 & 12.45 & 13.47 & 8.17 \\
\hline 127 & 12.63 & 13.82 & 9.38 \\
\hline 124 & 13.81 & 13.81 & 0.03 \\
\hline 119 & 14.96 & 13.82 & 7.57 \\
\hline 114 & 17.85 & 13.64 & 23.56 \\
\hline 109 & 12.19 & 13.64 & 11.89 \\
\hline 105 & 12.08 & 13.89 & 15.02 \\
\hline 94 & 15.51 & 16.65 & 7.32 \\
\hline 89 & 14.73 & 17.76 & 20.54 \\
\hline 84 & 19.61 & 18.62 & 5.02 \\
\hline 78 & 17.76 & 19.32 & 8.73 \\
\hline
\end{tabular}

Comparison of Oblique shock wave angles for Test 59

\begin{tabular}{|r|r|r|r|}
\hline Pressure Ratio & \multicolumn{2}{l|}{$\begin{array}{l}\text { Observed Angle } \\
(\mathrm{deg})\end{array}$} & $\begin{array}{l}\text { Calculated Angle } \\
(\mathrm{deg})\end{array}$ \\
\hline 84 & 20.19 & 14.82 & 26.59 \\
\hline 95 & 14.91 & 14.42 & 3.28 \\
\hline 103 & 16.73 & 14.19 & 15.20 \\
\hline 109 & 16.56 & 14.15 & 14.55 \\
\hline 113 & 17.08 & 13.87 & 18.81 \\
\hline 116 & 15.23 & 13.72 & 9.88 \\
\hline 122 & 14.48 & 13.45 & 7.09 \\
\hline 128 & 14.44 & 13.38 & 7.31 \\
\hline 124 & 13.86 & 13.45 & 2.92 \\
\hline 121 & 16.14 & 13.62 & 15.60 \\
\hline 117 & 15.15 & 13.78 & 9.00 \\
\hline 111 & 15.21 & 14.06 & 7.58 \\
\hline 105 & 17.38 & 14.43 & 17.01 \\
\hline 96 & 15.09 & 17.61 & 16.65 \\
\hline 89 & 14.45 & 18.32 & 26.77 \\
\hline 84 & 16.81 & 20.32 & 20.85 \\
\hline
\end{tabular}


Comparison of Oblique shock wave angles for Test 61

\begin{tabular}{|r|r|r|r|}
\hline Pressure Ratio & $\begin{array}{l}\text { Observed Angle } \\
(\mathrm{deg})\end{array}$ & $\begin{array}{l}\text { Calculated Angle } \\
(\mathrm{deg})\end{array}$ & \% Difference \\
\hline 81 & 17.94 & 14.96 & 16.61 \\
\hline 88 & 15.38 & 14.46 & 2.97 \\
\hline 94 & 14.64 & 14.25 & 11.67 \\
\hline 98 & 15.95 & 14.08 & 10.34 \\
\hline 104 & 15.37 & 13.78 & 1.52 \\
\hline 109 & 13.49 & 13.69 & 17.31 \\
\hline 113 & 16.50 & 13.64 & 3.69 \\
\hline 110 & 14.37 & 13.84 & 24.33 \\
\hline 102 & 18.42 & 13.94 & 18.92 \\
\hline 96 & 20.15 & 16.34 & 2.89 \\
\hline 91 & 18.01 & 18.53 & 29.57 \\
\hline 86 & 13.94 & 18.06 & 16.28 \\
\hline 80 & 17.02 & 19.80 & \\
\hline
\end{tabular}




\subsection{Appendix B}

Secondary Mach number comparison between CPSE simulation and test results

Test 52

\begin{tabular}{|c|c|c|c|}
\hline & \multicolumn{3}{|c|}{ Secondary Mach Number } \\
\hline PR & Test & Simulation & $\%$ difference \\
\hline 81 & 0.75 & 0.989 & 24.16 \\
\hline 91 & 0.79 & 0.989 & 20.12 \\
\hline 99 & 0.82 & 0.989 & 17.08 \\
\hline 105 & 0.83 & 0.949 & 12.53 \\
\hline 113 & 0.85 & 0.885 & 3.95 \\
\hline 119 & 0.86 & 0.83 & 3.61 \\
\hline 122 & 0.87 & 0.860 & 1.16 \\
\hline 123 & 0.86 & 0.846 & 1.65 \\
\hline 118 & 0.90 & 0.878 & 2.50 \\
\hline 112 & 0.92 & 0.891 & 3.25 \\
\hline 106 & 0.44 & 0.961 & 54.21 \\
\hline 99 & 0.33 & 0.989 & 66.63 \\
\hline 94 & 0.26 & 0.989 & 73.71 \\
\hline 88 & 0.22 & 0.989 & 77.75 \\
\hline
\end{tabular}

Test 53

\begin{tabular}{|c|c|c|c|}
\hline & \multicolumn{3}{|c|}{ Secondary Mach Number } \\
\hline PR & Test & Simulation & $\%$ difference \\
\hline 78 & 0.89 & 0.989 & 10.01 \\
\hline 88 & 0.95 & 0.989 & 3.94 \\
\hline 98 & 1.00 & 0.989 & 1.11 \\
\hline 104 & 0.99 & 0.931 & 6.33 \\
\hline 106 & 0.96 & 0.953 & 0.73 \\
\hline 103 & 0.92 & 0.935 & 1.60 \\
\hline 96 & 0.51 & 0.989 & 48.43 \\
\hline 91 & 0.41 & 0.989 & 58.54 \\
\hline 86 & 0.32 & 0.989 & 67.64 \\
\hline 81 & 0.25 & 0.989 & 74.72 \\
\hline
\end{tabular}


Test 54

\begin{tabular}{|c|c|c|c|}
\hline & \multicolumn{3}{|c|}{ Secondary Mach Number } \\
\hline PR & Test & Simulation & $\%$ difference \\
\hline 79 & 0.81 & 0.989 & 18.09 \\
\hline 88 & 0.84 & 0.989 & 15.06 \\
\hline 95 & 0.86 & 0.989 & 13.04 \\
\hline 104 & 0.89 & 0.944 & 5.72 \\
\hline 111 & 0.91 & 0.892 & 2.01 \\
\hline 116 & 0.89 & 0.860 & 3.48 \\
\hline 113 & 0.88 & 0.886 & 0.67 \\
\hline 107 & 0.89 & 0.902 & 1.33 \\
\hline 100 & 0.45 & 0.989 & 54.49 \\
\hline 94 & 0.36 & 0.989 & 63.59 \\
\hline 89 & 0.32 & 0.989 & 67.64 \\
\hline 83 & 0.27 & 0.989 & 72.69 \\
\hline 78 & 0.24 & 0.989 & 75.73 \\
\hline
\end{tabular}

Test 55

\begin{tabular}{|c|c|c|c|}
\hline & \multicolumn{3}{|c|}{ Secondary Mach Number } \\
\hline PR & Test & Simulation & $\%$ difference \\
\hline 79 & 0.88 & 0.989 & 11.02 \\
\hline 85 & 0.91 & 0.989 & 7.98 \\
\hline 89 & 0.93 & 0.989 & 5.96 \\
\hline 97 & 0.96 & 0.989 & 2.93 \\
\hline 102 & 0.98 & 0.989 & 0.91 \\
\hline 105 & 0.94 & 0.932 & 0.85 \\
\hline 102 & 0.94 & 0.989 & 4.95 \\
\hline 95 & 0.51 & 0.989 & 48.43 \\
\hline 90 & 0.40 & 0.989 & 59.55 \\
\hline 83 & 0.37 & 0.989 & 62.58 \\
\hline 78 & 0.31 & 0.989 & 68.65 \\
\hline
\end{tabular}


Test 56

\begin{tabular}{|c|c|c|c|}
\hline & \multicolumn{3}{|c|}{ Secondary Mach Number } \\
\hline PR & Test & Simulation & $\%$ difference \\
\hline 86 & 0.83 & 0.989 & 16.07 \\
\hline 94 & 0.85 & 0.989 & 14.05 \\
\hline 99 & 0.86 & 0.989 & 13.04 \\
\hline 103 & 0.87 & 0.959 & 9.28 \\
\hline 107 & 0.87 & 0.914 & 4.81 \\
\hline 113 & 0.87 & 0.886 & 1.80 \\
\hline 116 & 0.87 & 0.859 & 1.28 \\
\hline 113 & 0.86 & 0.885 & 2.82 \\
\hline 108 & 0.86 & 0.937 & 8.21 \\
\hline 103 & 0.86 & 0.930 & 7.52 \\
\hline 96 & 0.43 & 0.989 & 56.52 \\
\hline 90 & 0.36 & 0.989 & 63.59 \\
\hline 84 & 0.30 & 0.989 & 69.66 \\
\hline 79 & 0.20 & 0.989 & 79.77 \\
\hline
\end{tabular}

Test 57

\begin{tabular}{|r|r|r|r|}
\hline \multicolumn{1}{|l|}{ Secondary Mach Number } \\
\hline PR & Test & \multicolumn{1}{l|}{ Simulation } & \% difference \\
\hline 84 & 0.83 & 0.989 & 16.07 \\
\hline 93 & 0.85 & 0.989 & 14.05 \\
\hline 100 & 0.85 & 0.989 & 14.05 \\
\hline 105 & 0.86 & 0.956 & 10.04 \\
\hline 111 & 0.86 & 0.887 & 3.04 \\
\hline 118 & 0.85 & 0.868 & 2.07 \\
\hline 120 & 0.83 & 0.846 & 1.89 \\
\hline 118 & 0.82 & 0.864 & 5.09 \\
\hline 113 & 0.84 & 0.882 & 4.76 \\
\hline 108 & 0.84 & 0.918 & 8.49 \\
\hline 102 & 0.67 & 0.989 & 32.25 \\
\hline 96 & 0.36 & 0.989 & 63.59 \\
\hline 90 & 0.32 & 0.989 & 67.64 \\
\hline 85 & 0.33 & 0.989 & 66.63 \\
\hline
\end{tabular}


Test 58

\begin{tabular}{|r|r|r|r|}
\hline & \multicolumn{3}{|l|}{ Secondary Mach Number } \\
\hline PR & Test & \multicolumn{1}{l|}{ Simulation } & \% difference \\
\hline 84 & 0.85 & 0.989 & 14.05 \\
\hline 95 & 0.85 & 0.989 & 14.05 \\
\hline 103 & 0.85 & 0.946 & 10.14 \\
\hline 109 & 0.85 & 0.909 & 6.49 \\
\hline 118 & 0.83 & 0.878 & 5.46 \\
\hline 123 & 0.82 & 0.845 & 2.95 \\
\hline 127 & 0.80 & 0.780 & 2.56 \\
\hline 124 & 0.79 & 0.831 & 4.93 \\
\hline 119 & 0.81 & 0.840 & 3.57 \\
\hline 114 & 0.81 & 0.878 & 7.74 \\
\hline 109 & 0.82 & 0.909 & 9.79 \\
\hline 105 & 0.82 & 0.944 & 13.13 \\
\hline 94 & 0.31 & 0.989 & 68.65 \\
\hline 89 & 0.24 & 0.989 & 75.73 \\
\hline 84 & 0.23 & 0.989 & 76.74 \\
\hline 78 & 0.19 & 0.989 & 80.78 \\
\hline
\end{tabular}

Test 59

\begin{tabular}{|c|c|c|c|}
\hline & \multicolumn{3}{|c|}{ Secondary Mach Number } \\
\hline PR & Test & Simulation & $\%$ difference \\
\hline 84 & 0.92 & 0.989 & 6.97 \\
\hline 95 & 0.91 & 0.989 & 7.98 \\
\hline 103 & 0.89 & 0.947 & 6.01 \\
\hline 109 & 0.88 & 0.912 & 3.50 \\
\hline 113 & 0.87 & 0.881 & 1.24 \\
\hline 116 & 0.86 & 0.858 & 0.23 \\
\hline 122 & 0.84 & 0.812 & 3.44 \\
\hline 128 & 0.79 & 0.772 & 2.33 \\
\hline 124 & 0.80 & 0.827 & 3.26 \\
\hline 121 & 0.79 & 0.829 & 4.70 \\
\hline 117 & 0.79 & 0.895 & 11.73 \\
\hline 111 & 0.81 & 0.886 & 8.57 \\
\hline 105 & 0.82 & 0.911 & 9.98 \\
\hline 96 & 0.37 & 0.989 & 62.58 \\
\hline 89 & 0.29 & 0.989 & 70.67 \\
\hline 84 & 0.23 & 0.989 & 76.74 \\
\hline 78 & 0.14 & 0.989 & 85.84 \\
\hline
\end{tabular}




\section{Test 61}

\begin{tabular}{|r|r|r|r|}
\hline & \multicolumn{3}{|l|}{ Secondary Mach Number } \\
\hline PR & Test & \multicolumn{1}{l|}{ Simulation } & \% difference \\
\hline 81 & 0.80 & 0.989 & 19.11 \\
\hline 88 & 0.83 & 0.989 & 16.07 \\
\hline 94 & 0.85 & 0.989 & 14.05 \\
\hline 98 & 0.87 & 0.989 & 12.03 \\
\hline 104 & 0.90 & 0.951 & 5.36 \\
\hline 109 & 0.90 & 0.902 & 0.22 \\
\hline 113 & 0.88 & 0.882 & 0.22 \\
\hline 110 & 0.87 & 0.922 & 5.63 \\
\hline 102 & 0.63 & 0.989 & 36.29 \\
\hline 96 & 0.49 & 0.989 & 50.45 \\
\hline 91 & 0.64 & 0.989 & 35.28 \\
\hline 86 & 0.49 & 0.989 & 50.45 \\
\hline 80 & 0.43 & 0.989 & 56.52 \\
\hline
\end{tabular}




\subsection{Appendix C}

Comparison of Fabri choke location, Oblique shock starting location and CPSE simulation

Test 52

\begin{tabular}{|c|c|c|c|c|c|}
\hline \multirow[t]{2}{*}{ PR } & \multirow{2}{*}{$\begin{array}{l}\text { Fabri Choke } \\
\text { location (in) }\end{array}$} & \multirow{2}{*}{$\begin{array}{l}\text { Location of } \\
\text { Oblique Shock } \\
\text { (in) }\end{array}$} & \multirow{2}{*}{$\begin{array}{l}\text { Flow turning } \\
\text { point (in) }\end{array}$} & \multicolumn{2}{|c|}{ Plume Expansion (in) } \\
\hline & & & & Test & Simulation \\
\hline 105 & 20.67 & 1.460 & 1.716 & 0.760 & 0.726 \\
\hline 113 & 3.70 & 1.434 & 1.720 & 0.756 & 0.732 \\
\hline 119 & 2.61 & 1.687 & 1.725 & 0.792 & 0.740 \\
\hline 122 & 2.01 & 1.532 & 1.738 & 0.790 & 0.736 \\
\hline 123 & 2.02 & 1.540 & 1.739 & 0.796 & 0.739 \\
\hline 118 & 1.68 & 1.504 & 1.728 & 0.814 & 0.733 \\
\hline 112 & 1.87 & 1.364 & 1.724 & 0.652 & 0.731 \\
\hline 106 & -2.12 & 1.123 & 1.714 & 0.608 & 0.726 \\
\hline
\end{tabular}

Test 53

\begin{tabular}{|c|c|c|c|c|c|}
\hline \multirow[t]{2}{*}{ PR } & \multirow{2}{*}{$\begin{array}{l}\text { Fabri Choke } \\
\text { location (in) }\end{array}$} & \multirow{2}{*}{$\begin{array}{l}\text { Location of } \\
\text { Oblique Shock } \\
\text { (in) }\end{array}$} & \multirow{2}{*}{$\begin{array}{l}\text { Flow turning } \\
\text { point (in) }\end{array}$} & \multicolumn{2}{|c|}{ Plume Expansion (in) } \\
\hline & & & & Test & Simulation \\
\hline 104 & 0.64 & 1.577 & 1.711 & 0.754 & 0.727 \\
\hline 106 & 0.76 & 1.394 & 1.714 & 0.730 & 0.726 \\
\hline 103 & 1.13 & 1.439 & 1.711 & 0.717 & 0.727 \\
\hline
\end{tabular}

Test 54

\begin{tabular}{|c|c|c|c|c|c|}
\hline \multirow[t]{2}{*}{ PR } & \multirow{2}{*}{$\begin{array}{l}\text { Fabri Choke } \\
\text { location (in) }\end{array}$} & \multirow{2}{*}{$\begin{array}{l}\text { Location of } \\
\text { Oblique Shock } \\
\text { (in) }\end{array}$} & \multirow{2}{*}{$\begin{array}{l}\text { Flow turning } \\
\text { point (in) }\end{array}$} & \multicolumn{2}{|c|}{ Plume Expansion (in) } \\
\hline & & & & Test & Simulation \\
\hline 104 & 4.70 & 1.636 & 1.715 & 0.791 & 0.727 \\
\hline 111 & 2.53 & 1.504 & 1.724 & 0.761 & 0.731 \\
\hline 116 & 1.61 & 1.350 & 1.723 & 0.795 & 0.736 \\
\hline 113 & 1.64 & 1.331 & 1.720 & 0.790 & 0.732 \\
\hline 107 & 1.59 & 1.357 & 1.719 & 0.796 & 0.730 \\
\hline
\end{tabular}


Test 55

\begin{tabular}{|c|c|c|c|c|c|}
\hline \multirow[t]{2}{*}{ PR } & \multirow{2}{*}{$\begin{array}{l}\text { Fabri Choke } \\
\text { location (in) }\end{array}$} & \multirow{2}{*}{$\begin{array}{l}\text { Location of } \\
\text { Oblique Shock } \\
\text { (in) }\end{array}$} & \multirow{2}{*}{$\begin{array}{l}\text { Flow turning } \\
\text { point (in) }\end{array}$} & \multicolumn{2}{|c|}{ Plume Expansion (in) } \\
\hline & & & & Test & Simulation \\
\hline 102 & 0.40 & 1.397 & 1.711 & 0.761 & 0.725 \\
\hline 105 & 0.82 & 1.444 & 1.716 & 0.770 & 0.727 \\
\hline 102 & 0.97 & 1.460 & 1.710 & 0.724 & 0.724 \\
\hline
\end{tabular}

Test 56

\begin{tabular}{|c|c|c|c|c|c|}
\hline \multirow[t]{2}{*}{ PR } & \multirow{2}{*}{$\begin{array}{l}\text { Fabri Choke } \\
\text { location (in) }\end{array}$} & \multirow{2}{*}{$\begin{array}{l}\text { Location of } \\
\text { Oblique Shock } \\
\text { (in) }\end{array}$} & \multirow{2}{*}{$\begin{array}{l}\text { Flow turning } \\
\text { point (in) }\end{array}$} & \multicolumn{2}{|c|}{ Plume Expansion (in) } \\
\hline & & & & Test & Simulation \\
\hline 103 & 3.59 & 1.500 & 1.714 & 0.785 & 0.726 \\
\hline 107 & 2.57 & 1.514 & 1.719 & 0.777 & 0.729 \\
\hline 113 & 2.03 & 1.511 & 1.720 & 0.778 & 0.732 \\
\hline 116 & 1.71 & 1.691 & 1.724 & 0.763 & 0.736 \\
\hline 113 & 1.58 & 1.493 & 1.720 & 0.761 & 0.732 \\
\hline 108 & 1.74 & 1.514 & 1.716 & 0.726 & 0.727 \\
\hline 103 & 1.46 & 1.406 & 1.714 & 0.709 & 0.728 \\
\hline
\end{tabular}

Test 57

\begin{tabular}{|r|r|r|r|r|r|}
\hline PR & $\begin{array}{l}\text { Fabri Choke } \\
\text { location (in) }\end{array}$ & \multicolumn{2}{l|}{$\begin{array}{l}\text { Location of } \\
\text { Oblique Shock } \\
\text { (in) }\end{array}$} & $\begin{array}{l}\text { Flow turning } \\
\text { point (in) }\end{array}$ & \multicolumn{2}{l|}{ Plume Expansion (in) } \\
\cline { 5 - 6 } & 2.61 & 1.625 & 1.715 & 0.745 & \multicolumn{2}{l|}{ Test } \\
\hline 105 & 2.05 & 1.511 & 1.726 & 0.798 & 0.726 \\
\hline 113 & 1.76 & 1.714 & 1.732 & 0.776 & 0.735 \\
\hline 119 & 1.77 & 1.738 & 1.735 & 0.785 & 0.739 \\
\hline 122 & 1.82 & 1.702 & 1.732 & 0.756 & 0.735 \\
\hline 123 & 1.73 & 1.759 & 1.720 & 0.733 & 0.733 \\
\hline 118 & 1.63 & 1.713 & 1.722 & 0.712 & 0.728 \\
\hline
\end{tabular}


Test 58

\begin{tabular}{|c|c|c|c|c|c|}
\hline \multirow[t]{2}{*}{ PR } & \multirow{2}{*}{$\begin{array}{l}\text { Fabri Choke } \\
\text { location (in) }\end{array}$} & \multirow{2}{*}{$\begin{array}{l}\text { Location of } \\
\text { Oblique Shock } \\
\text { (in) }\end{array}$} & \multirow{2}{*}{$\begin{array}{l}\text { Flow turning } \\
\text { point (in) }\end{array}$} & \multicolumn{2}{|c|}{ Plume Expansion (in) } \\
\hline & & & & Test & Simulation \\
\hline 103 & 3.51 & 1.599 & 1.714 & 0.724 & 0.726 \\
\hline 109 & 2.61 & 1.498 & 1.718 & 0.751 & 0.729 \\
\hline 118 & 2.4 & 1.738 & 1.728 & 0.742 & 0.733 \\
\hline 123 & 2.13 & 1.634 & 1.739 & 0.760 & 0.739 \\
\hline 127 & 2.23 & 1.700 & 1.737 & 0.763 & 0.754 \\
\hline 124 & 2.15 & 1.761 & 1.739 & 0.729 & 0.741 \\
\hline 119 & 1.96 & 1.493 & 1.732 & 0.791 & 0.740 \\
\hline 114 & 1.96 & 1.584 & 1.728 & 0.747 & 0.733 \\
\hline 109 & 1.77 & 1.457 & 1.718 & 0.716 & 0.729 \\
\hline 105 & 1.71 & 1.411 & 1.715 & 0.686 & 0.726 \\
\hline
\end{tabular}

Test 59

\begin{tabular}{|c|c|c|c|c|c|}
\hline \multirow[t]{2}{*}{ PR } & \multirow{2}{*}{$\begin{array}{l}\text { Fabri Choke } \\
\text { location (in) }\end{array}$} & \multirow{2}{*}{$\begin{array}{l}\text { Location of } \\
\text { Oblique Shock } \\
\text { (in) }\end{array}$} & \multirow{2}{*}{$\begin{array}{l}\text { Flow turning } \\
\text { point (in) }\end{array}$} & \multicolumn{2}{|c|}{ Plume Expansion (in) } \\
\hline & & & & Test & Simulation \\
\hline 103 & 1.6 & 1.441 & 1.714 & 0.796 & 0.726 \\
\hline 109 & 1.73 & 1.383 & 1.722 & 0.796 & 0.729 \\
\hline 113 & 1.8 & 1.504 & 1.720 & 0.804 & 0.733 \\
\hline 116 & 1.89 & 1.434 & 1.724 & 0.809 & 0.736 \\
\hline 122 & 2.07 & 1.430 & 1.730 & 0.809 & 0.746 \\
\hline 128 & 2.32 & 1.468 & 1.739 & 0.776 & 0.756 \\
\hline 124 & 2.22 & 1.742 & 1.738 & 0.765 & 0.742 \\
\hline 121 & 2.23 & 1.478 & 1.735 & 0.757 & 0.742 \\
\hline 117 & 2.24 & 1.699 & 1.724 & 0.807 & 0.731 \\
\hline 111 & 2.17 & 1.710 & 1.726 & 0.840 & 0.732 \\
\hline 105 & 2.03 & 1.341 & 1.719 & 0.820 & 0.729 \\
\hline
\end{tabular}

Test 61

\begin{tabular}{|c|c|c|c|c|c|}
\hline \multirow[t]{2}{*}{ PR } & \multirow{2}{*}{$\begin{array}{l}\text { Fabri Choke } \\
\text { location (in) }\end{array}$} & \multirow{2}{*}{$\begin{array}{l}\text { Location of } \\
\text { Oblique Shock } \\
\text { (in) }\end{array}$} & \multirow{2}{*}{$\begin{array}{l}\text { Flow turning } \\
\text { point (in) }\end{array}$} & \multicolumn{2}{|c|}{ Plume Expansion (in) } \\
\hline & & & & Test & Simulation \\
\hline 104 & 1.74 & 1.514 & 1.714 & 0.779 & 0.726 \\
\hline 109 & 1.44 & 1.590 & 1.718 & 0.790 & 0.730 \\
\hline 113 & 1.58 & 1.580 & 1.720 & 0.818 & 0.733 \\
\hline 110 & 1.53 & 1.533 & 1.718 & 0.763 & 0.728 \\
\hline 102 & 1.21 & 1.336 & 1.706 & 0.706 & 0.722 \\
\hline
\end{tabular}

